\title{
WEIGHT-MONODROMY CONJECTURE FOR $p$-ADICALLY UNIFORMIZED VARIETIES
}

\author{
TETSUSHI ITO
}

\begin{abstract}
The aim of this paper is to prove the weight-monodromy conjecture (Deligne's conjecture on the purity of monodromy filtration) for varieties $p$-adically uniformized by the Drinfeld upper half spaces of any dimension. The ingredients of the proof are to prove a special case of the Hodge standard conjecture, and apply a positivity argument of Steenbrink, M. Saito to the weight spectral sequence of Rapoport-Zink. As an application, by combining our results with the results of Schneider-Stuhler, we compute the local zeta functions of $p$-adically uniformized varieties in terms of representation theoretic invariants. We also consider a $p$-adic analogue by using the weight spectral sequence of Mokrane.
\end{abstract}

\section{INTRODUCTION}

Let $K$ be a complete discrete valuation field with finite residue field $\mathbb{F}_{q}$ of characteristic $p>0, \mathscr{O}_{K}$ the ring of integers of $K$, and $l$ a prime number different from $p$. Let $X$ be a proper smooth variety over $K$, and $V:=H_{\text {êt }}^{w}\left(X_{\bar{K}}, \mathbb{Q}_{l}\right)$ the $l$ adic cohomology of $X_{\bar{K}}:=X \otimes_{K} \bar{K}$ on which the absolute Galois group Gal $(\bar{K} / K)$ acts. We define the inertia group $I_{K}$ of $K$ by the exact sequence:

$$
1 \longrightarrow I_{K} \longrightarrow \operatorname{Gal}(\bar{K} / K) \longrightarrow \operatorname{Gal}\left(\overline{\mathbb{F}}_{q} / \mathbb{F}_{q}\right) \longrightarrow 1 \text {. }
$$

The monodromy filtration $M_{\bullet}$ on $V$ is determined by the action of $I_{K}$ on $V$, and the weight filtration $W \bullet$ on $V$ is determined by the action of a lift of the geometric Frobenius element $\operatorname{Fr}_{q} \in \operatorname{Gal}\left(\overline{\mathbb{F}}_{q} / \mathbb{F}_{q}\right)$, which is the inverse of the $q$-th power map on $\overline{\mathbb{F}}_{q}$ (for details, see Definition [5.1] Definition [5.3).

The weight-monodromy conjecture claims the coincidence of these two filtrations up to some shift ([De1, [De3, [11], [12], [13], [RZ1, Rap ). Sometimes, the weight-monodromy conjecture is also called Deligne's conjecture on the purity of monodromy filtration in the literature.

Conjecture 1.1 (Weight-monodromy conjecture).

$$
M_{i} V=W_{i+w} V \quad \text { for all } i .
$$

Date: October 25, 2018.

1991 Mathematics Subject Classification. Primary: 11G25; Secondary: 11F80, 11F85, 14G20, 14F20. 
Let us briefly recall the theory of $p$-adic uniformization by Tate, Raynaud, Mumford, Mustafin, Kurihara, and others ([Mum, [Mus, $[\mathrm{Ku}]$ ). Let $\widehat{\Omega}_{K}^{d}$ be the Drinfeld upper half space of dimension $d \geq 1$, which is a rigid analytic space obtained by removing all $K$-rational hyperplanes from $\mathbb{P}_{K}^{d}$. We have a natural action of $\mathrm{PGL}_{d+1}(K)$ on $\widehat{\Omega}_{K}^{d}$. For a cocompact torsion free discrete subgroup $\Gamma \subset \mathrm{PGL}_{d+1}(K)$, the rigid analytic quotient $\Gamma \backslash \widehat{\Omega}_{K}^{d}$ has a structure of a projective smooth variety $X_{\Gamma}$ over $K$ (for details, see 96 ). The main theorem of this paper is as follows.

Theorem 1.2 (Weight-monodromy conjecture for $X_{\Gamma}$ ). Let $\Gamma \subset \mathrm{PGL}_{d+1}(K)$ be a cocompact torsion free discrete subgroup. Then, the weight-monodromy conjecture (Conjecture 1.1) holds for $X_{\Gamma}$.

Note that Conjecture 1.1 was known to hold if $X / K$ satisfies at least one of the following conditions.

(1) $X$ has a proper smooth model over $\mathscr{O}_{K}$ (SGA4-III, De2, De3]).

(2) $X$ is a curve or an abelian variety over $K$ ([SGA7-I], IX).

(3) $X$ is a surface ([RZ1 for the semistable case, dJ] for the general case).

(4) $X$ is a certain threefold with strictly semistable reduction ([It2]).

(5) $K$ is of characteristic $p>0$ ([De3], [Te, [t] $)$.

Therefore, Theorem 1.2 is new only in mixed characteristic although our proof of Theorem 1.2 is valid even if $K$ is of characteristic $p>0$ (However, see also Remark 6.2). In mixed characteristic and in dimension $\geq 3$, Conjecture 1.1 is still open up to now. Therefore, Theorem 1.2 gives us an interesting class of varieties of any dimension in mixed characteristic for which Conjecture 1.1 holds.

Our proof of Theorem 1.2 is based on a careful analysis of the weight spectral sequence of Rapoport-Zink ([RZ1] ). We prove a special case of the Hodge standard conjecture and use it to follow a positivity argument of Steenbrink, M. Saito in [St], SaM1, where they proved a Hodge analogue of Conjecture 1.1] by using polarized Hodge structures (see also [GNA]). Namely, in our proof of Theorem 1.2 the Hodge standard conjecture plays a role of polarized Hodge structures. Note that in [It2], by basically the same idea as in this paper, the author proved Conjecture 1.1 for certain threefolds by using the Hodge index theorem which is nothing but the Hodge standard conjecture for surfaces.

The outline of this paper is as follows. In \$2, we fix notation and recall some basic facts about the Hodge standard conjecture and related theorems. Note that, in this paper, we mainly consider varieties whose $l$-adic cohomology groups are generated by algebraic cycles. We introduce appropriate notation in $\$ 2.1$.

In 93 , we recall some properties of blowing-up and prove the Hodge standard conjecture of blow-ups under certain assumptions by looking at the asymptotic behavior of the Lefschetz operator and the cup product pairings (Proposition 3.4). Such argument seems well-known at least in the simplest case of a blow-up 
at a point on a surface. However, since the multiplicative structure of a blow-up is not so simple in higher dimensions, we need tedious computations to prove it.

In $₫$, we consider a projective smooth variety $B^{n}$ of dimension $n$ over $\overline{\mathbb{F}}_{q}$ which is obtained by successive blowing-ups of $\mathbb{P}_{\mathbb{F}_{q}}^{n}$ along linear subvarieties. The main result in 4 is a proof of the Hodge standard conjecture for such varieties for certain choice of an ample line bundle (Proposition 4.7). Although we can, in principle, compute everything combinatorially about the variety $B^{n}$, the proof of Proposition 4.7 is far from trivial. We look at the combinatorial structure of $B^{n}$ very carefully, and the proof proceeds by induction on $n$. This proof is inspired by McMullen's proof of an analogue of the hard Lefschetz theorem for non-rational polytopes $([\mathrm{Mc}])$.

In \$5, we recall some basic facts on the weight spectral sequence of RapoportZink. By a positivity argument of Steenbrink, M. Saito, we give a proof of a special case of Conjecture 1.1] assuming the existence of $\mathbb{Q}$-structures on $l$-adic cohomology and the Hodge standard conjecture (Proposition 5.7).

In $\$ 6$, we firstly give a proof of Theorem 1.2. The key point is that there is an explicitly constructed formal scheme $\widehat{\Omega}_{\mathscr{O}_{K}}^{d}$ locally of finite type over $\operatorname{Spf} \mathscr{O}_{K}$, whose associated rigid analytic space is $\widehat{\Omega}_{K}^{d}$. From the construction, we see that $X_{\Gamma}$ has a proper semistable model $\mathfrak{X}_{\Gamma}$ over $\mathscr{O}_{K}$. Moreover, if $\mathfrak{X}_{\Gamma}$ is strictly semistable, we see that all irreducible components of the special fiber of $\mathfrak{X}_{\Gamma}$ are isomorphic to the variety $B^{d}$ in $\$ 4$. Hence we can prove the Hodge standard conjecture for them by Proposition 4.7. The same is true for intersections of them. By Proposition 5.7 the proof of Theorem 1.2 is complete.

In the rest of $\$ 6$ and $\$ 7$, we also give some applications and complements. By combining Theorem 1.2 with the results of Schneider-Stuhler on the $l$-adic cohomology of $X_{\Gamma}(\underline{\mathrm{SS}}]$ ), we give a proof of Schneider-Stuhler's conjecture on the filtration $F^{\bullet}$ on $H_{\text {ét }}^{d}\left(X_{\bar{K}}, \mathbb{Q}_{l}\right)$ (Theorem 6.3). As a consequence, we prove the following formula of the local zeta function $\zeta\left(s, X_{\Gamma}\right)$ of $X_{\Gamma}$ :

$$
\begin{aligned}
\zeta\left(s, X_{\Gamma}\right) & :=\prod_{k=0}^{2 d} \operatorname{det}\left(1-q^{-s} \cdot \operatorname{Fr}_{q} ; H_{\text {ét }}^{k}\left(X_{\Gamma} \otimes_{K} \bar{K}, \mathbb{Q}_{l}\right)^{I_{K}}\right)^{(-1)^{k+1}} \\
& =\left(1-q^{-s}\right)^{\mu(\Gamma) \cdot(-1)^{d+1}} \cdot \prod_{k=0}^{d} \frac{1}{1-q^{k-s}}
\end{aligned}
$$

where $\mu(\Gamma)$ is the multiplicity of the Steinberg representation in the representation of $\mathrm{PGL}_{d+1}(K)$ induced from the trivial character on $\Gamma$ (Theorem 6.4). In particular, $\zeta\left(s, X_{\Gamma}\right)$ is independent of $l$. In 6.5 , we consider a $p$-adic analogue by using the weight spectral sequence of Mokrane ([Mo $)$. After proving a $p$-adic analogue of Conjecture 1.1 for $X_{\Gamma}$, we also prove that the local zeta function $\zeta_{p \text {-adic }}\left(s, X_{\Gamma}\right)$ defined via $p$-adic Hodge theory coincides with $\zeta\left(s, X_{\Gamma}\right)$ above (Theorem 6.8. Theorem 6.10). Finally, in $₫ 7$, we give an application of these 
results to the Tate conjecture for varieties over number fields admitting $p$-adic uniformization.

Needless to say, there is a very important theory so called the theory of $p$ adic uniformization of Shimura varieties established by Čerednik, Drinfeld for Shimura curves, and Rapoport-Zink, Varshavsky in higher dimensions ( $\check{\mathrm{C}}$, $[\mathrm{Dr}$, [RZ2, Va] ). We expect that Theorem 1.2 has applications to the zeta functions of Shimura varieties admitting $p$-adic uniformization ( Rap, [RZ1]). We also expect that the results of this paper will establish a special case of the compatibility between the global and the local Langlands correspondences ([Harr], Problem 1).

Remark 1.3. After the first version of this paper was written, Ehud de Shalit informed that he obtained a $p$-adic analogue of Conjecture 1.1 for $X_{\Gamma}$ (Theorem [6.8) by a completely different method. His proof relies on a combinatorial result of Alon-de Shalit about harmonic cochains on the Bruhat-Tits buildings ([AdS, dS $)$. Wayne Raskind informed that he and Xavier Xarles studied the cohomology of varieties with "totally degenerate reduction" ([RX]). Because of a special case of the Hodge standard conjecture proved in this paper, $p$-adically uniformized varieties are examples of varieties with "totally degenerate reduction" in the sense of Raskind-Xarles.

Acknowledgments. In the preparation of this paper, the author benefited from many people. Especially, the final part of this work was done in Arbeitsgemeinschaft Algebraische Geometrie at Universität zu Köln in November and December 2002. The author is pleased to thank Michael Rapoport for his continuous advice, encouragements, and discussions. He also informed that Theorem 1.2 has an application to the Tate conjecture for varieties admitting $p$-adic uniformization. His result is included in \$7] with his permission. The author also would like to thank Gerd Faltings, Michael Harris, Luc Illusie, Fumiharu Kato, Kazuya Kato, Minhyong Kim, Barry Mazur, Yoichi Mieda, Yukiyoshi Nakkajima, Richard Taylor, and Teruyoshi Yoshida for invaluable discussions and comments. Finally, the author is grateful to his advisor Professor Takeshi Saito for advice and support during the graduate course at the University of Tokyo. A part of this work was done during the author's stay at Universität zu Köln in June 2002, Université de Paris-Sud in June 2002, and Max-Planck-Institut für Mathematik in Bonn from November 2002 to October 2003. The author would like to thank all of them for their support and cordial hospitality. The author was supported by the Japan Society for the Promotion of Science Research Fellowships for Young Scientists.

\section{Review of the Hodge standard COnjeCture}

2.1. Notation and assumptions. In this paper, a variety means a separated scheme of finite type over a field which is geometrically reduced. We do not assume it is geometrically irreducible. Let $F$ be an algebraically closed field of 
any characteristic, $l$ a prime number different from the characteristic of $F$. Let $X$ be a projective smooth variety over $F$ of dimension $n$. Hence $X$ is a disjoint union of irreducible varieties of dimension $n$. Let $Z^{k}(X)$ be the group of algebraic cycles on $X$ of codimension $k$, and $\mathrm{cl}^{k}: Z^{k}(X) \rightarrow H_{\text {ét }}^{2 k}\left(X, \mathbb{Q}_{l}(k)\right)$ the cycle map for $l$-adic cohomology. Let $Z_{\text {num }}^{k}(X) \subset Z^{k}(X)$ be the subgroup consisting of algebraic cycles which are numerically equivalent to zero. It is known that

$$
N^{k}(X):=Z^{k}(X) / Z_{\text {num }}^{k}(X)
$$

is a finitely generated free $\mathbb{Z}$-module ([K] , Lemma 5-2, see also Lemma 2.3 below). Here we put the following assumption on $X$.

\section{Assumption 2.1.}

$$
\operatorname{dim}_{\mathbb{Q}_{l}} H_{\text {ét }}^{k}\left(X, \mathbb{Q}_{l}\right)= \begin{cases}\operatorname{rank}_{\mathbb{Z}} N^{k / 2}(X) & \text { if } k \text { is even } \\ 0 & \text { if } k \text { is odd. }\end{cases}
$$

Remark 2.2. Assumption 2.1 is independent of $l$ because the $l$-adic Betti numbers are independent of $l$ (This is a consequence of the Weil conjecture, see, for example, [KM], Corollary 1).

Lemma 2.3. Under Assumption [2.1, $\mathrm{cl}^{k}$ induces an isomorphism:

$$
N^{k}(X) \otimes_{\mathbb{Z}} \mathbb{Q}_{l} \cong H_{\text {ét }}^{2 k}\left(X, \mathbb{Q}_{l}(k)\right) \text {. }
$$

Proof. Fix an integer $k$. Let $V \subset H_{\text {ét }}^{2 k}\left(X, \mathbb{Q}_{l}(k)\right)$ be a $\mathbb{Q}_{l}$-vector subspace generated by the image of the cycle map cl $\mathrm{c}^{k}$. Take elements $x_{1}, \ldots, x_{r} \in Z^{k}(X)$ such that $\left\{\mathrm{cl}^{k}\left(x_{1}\right), \ldots, \mathrm{cl}^{k}\left(x_{r}\right)\right\}$ is a basis of $V$ over $\mathbb{Q}_{l}$. Then, it is easy to see that the image of the map

$$
Z^{n-k}(X) \longrightarrow \mathbb{Z}^{\oplus r}, \quad y \mapsto\left(y \cdot x_{1}, \ldots, y \cdot x_{r}\right)
$$

is isomorphic to $N^{n-k}(X)$, where - denotes the intersection product. Hence we have the following inequalities:

$$
\operatorname{rank}_{\mathbb{Z}} N^{n-k}(X) \leq r \leq \operatorname{dim}_{\mathbb{Q}_{l}} H_{\text {ét }}^{2 k}\left(X, \mathbb{Q}_{l}\right)=\operatorname{dim}_{\mathbb{Q}_{l}} H_{\text {ét }}^{2 n-2 k}\left(X, \mathbb{Q}_{l}\right) .
$$

Therefore, Assumption 2.1 implies that the above inequalities are equalities. Hence $V=H_{\text {ét }}^{2 k}\left(X, \mathbb{Q}_{l}(k)\right)$ for all $k$, and all cohomology classes are generated by algebraic cycles. In particular, the numerical equivalence coincides with the $l$-adic homological equivalence. We have a surjective map $N^{k}(X) \otimes_{\mathbb{Z}} \mathbb{Q}_{l} \rightarrow$ $H_{\text {ét }}^{2 k}\left(X, \mathbb{Q}_{l}(k)\right)$, between $\mathbb{Q}_{l}$-vector spaces of the same dimension, hence it is an isomorphism.

Namely, $N^{k}(X)$ defines $l$-independent $\mathbb{Q}$-structures on the $l$-adic cohomology of $X$. Similarly, by using the cycle map for crystalline cohomology (GM], Gr]), it is easy to see that $N^{k}(X)$ also defines $\mathbb{Q}$-structures on the crystalline cohomology of $X$. 
We define

$$
H^{k}(X):= \begin{cases}N^{k / 2}(X) \otimes_{\mathbb{Z}} \mathbb{R} & \text { if } k \text { is even } \\ 0 & \text { if } k \text { is odd, }\end{cases}
$$

and $H^{*}(X):=\bigoplus_{k} H^{k}(X)$. We consider $H^{*}(X)$ virtually as cohomology with coefficients in $\mathbb{R}$. The cup product $\cup$ on $H^{*}(X)$ is induced by the intersection product. Most properties of $l$-adic cohomology such as pullback, Poincaré duality, Künneth formula, etc. are valid for $H^{*}(X)$. If $X$ is irreducible, we have $H^{0}(X)=$ $H^{2 n}(X)=\mathbb{R}$ as usual. To avoid confusion, we always put the subscript "ét" for étale cohomology.

A $\mathbb{Q}$-divisor (resp. $\mathbb{R}$-divisor) is a formal sum of divisors with coefficients in $\mathbb{Q}(\operatorname{resp} . \mathbb{R})$. A $\mathbb{Q}$-divisor (resp. $\mathbb{R}$-divisor) $L$ is called ample if $L$ is of the form $L=a_{1} L_{1}+\cdots+a_{r} L_{r}$ with $r \geq 1, a_{1}, \ldots, a_{r} \in \mathbb{Q}_{>0}\left(\right.$ resp. $\left.\mathbb{R}_{>0}\right)$, and $L_{1}, \ldots, L_{r}$ ample. If $L$ is an ample $\mathbb{Q}$-divisor, some positive integral multiple of $L$ is an ample divisor. But this does not hold for ample $\mathbb{R}$-divisors.

There is a natural map from the group of $\mathbb{R}$-divisors on $X$ to $H^{2}(X)$. In this paper, we do not usually distinguish an $\mathbb{R}$-divisor, a formal sum of line bundles with coefficients in $\mathbb{R}$ (i.e. $\mathbb{R}$-line bundle), and its class in $H^{2}(X)$. We can naturally define pullbacks and restrictions for $\mathbb{R}$-divisors. To avoid confusion, for an $\mathbb{R}$-divisor $H$, we sometimes use the notation $\mathscr{O}(H)$ instead of $H$. For example, $f^{*} \mathscr{O}(H)$ (resp. $\left.\mathscr{O}(H)\right|_{X}$ ) denotes the pullback (resp. restriction) as a formal sum of line bundles.

2.2. Hard Lefschetz conjecture. As in \$2.1, let $X$ be a projective smooth variety over $F$ of dimension $n$ satisfying Assumption 2.1] and $L$ an ample $\mathbb{R}$ divisor on $X$. By taking cup product with $L$, we have an $\mathbb{R}$-linear map

$$
L: H^{k}(X) \longrightarrow H^{k+2}(X)
$$

called the Lefschetz operator.

Conjecture 2.4 (Hard Lefschetz conjecture). For all $k, L^{k}$ induces an isomorphism:

$$
L^{k}: H^{n-k}(X) \stackrel{\cong}{\longrightarrow} H^{n+k}(X)
$$

If $L$ is a $\mathbb{Q}$-divisor, Conjecture 2.4 is proved by Deligne in all characteristics ([De3 $)$. If $F=\mathbb{C}$, Conjecture 2.4 holds by transcendental methods (We $)$. The general characteristic 0 case follows from this by Lefschetz principle. However, in characteristic $p>0$, Conjecture 2.4 seems open in general.

Assume that Conjecture 2.4 holds for $(X, L)$. For an integer $k$ with $0 \leq k \leq n$, we define the primitive part $P^{k}(X)$ by

$$
P^{k}(X)=\operatorname{Ker}\left(L^{n-k+1}: H^{k}(X) \longrightarrow H^{2 n-k+2}(X)\right) .
$$


We define $P^{k}(X)=0$ for $k<0$ or $k>n$. Then, by Conjecture 2.4, we have the primitive decomposition:

$$
H^{k}(X)=\bigoplus_{i \geq 0} L^{i} P^{k-2 i}(X) \cong \bigoplus_{i \geq 0} P^{k-2 i}(X) \text { for all } k
$$

Let $X_{1}, \ldots, X_{m}$ be the irreducible components of $X$. Then we have a natural summation map

$$
\begin{aligned}
H^{2 n}(X)=\bigoplus_{i=1}^{m} H^{2 n}\left(X_{i}\right)=\bigoplus_{i=1}^{m} \mathbb{R} & \longrightarrow \mathbb{R} \\
\left(a_{1}, \ldots, a_{m}\right) & \mapsto a_{1}+\cdots+a_{m}
\end{aligned}
$$

which we denote by $\sigma$. For even $k$ with $0 \leq k \leq n$, we define the pairing $\langle,\rangle_{H^{k}(X)}$ on $H^{k}(X)$ by the composite of the following maps:

$$
\begin{aligned}
& \langle,\rangle_{H^{k}(X)}: H^{k}(X) \times H^{k}(X) \quad \longrightarrow \quad H^{2 n}(X) \stackrel{\sigma}{\longrightarrow} \mathbb{R} \\
& (x, y) \mapsto(-1)^{k / 2} L^{n-k} x \cup y .
\end{aligned}
$$

Otherwise, we define $\langle,\rangle_{H^{k}(X)}$ to be the zero pairing. The pairings $\langle,\rangle_{H^{k}(X)}$ are nondegenerate by Conjecture 2.4 and Poincaré duality.

We denote the restriction of $\langle,\rangle_{H^{k}(X)}$ to $P^{k}(X)$ by $\langle,\rangle_{P^{k}(X)}$. The decomposition (2.1) is an orthogonal decomposition with respect to $\langle,\rangle_{H^{k}(X)}$. Therefore, each $\langle,\rangle_{P^{k}(X)}$ is nondegenerate. Moreover, for all $k$ with $0 \leq k \leq n,\langle,\rangle_{H^{k}(X)}$ is isomorphic to the alternating sum of $\langle,\rangle_{P^{k-2 i}(X)}$ for $i \geq 0$ :

$$
\langle,\rangle_{H^{k}(X)}=\sum_{i \geq 0}(-1)^{i}\langle,\rangle_{P^{k-2 i}(X)} \quad \text { on } \quad H^{k}(X) \cong \bigoplus_{i \geq 0} P^{k-2 i}(X) .
$$

Remark 2.5. The primitive decomposition may depend on the choice of $L$. However, the dimensions of $P^{k}(X)$ are independent of $L$ because

$$
\operatorname{dim}_{\mathbb{R}} P^{k}(X)=\operatorname{dim}_{\mathbb{R}} H^{k}(X)-\operatorname{dim}_{\mathbb{R}} H^{k-2}(X) .
$$

2.3. Hodge standard conjecture. As in $\$ 2.2$ let $X$ be a projective smooth variety over $F$ of dimension $n$ satisfying Assumption 2.1 , and $L$ an ample $\mathbb{R}$ divisor on $X$. Assume further that the hard Lefschetz conjecture (Conjecture 2.4) holds for $(X, L)$.

Conjecture 2.6 (Hodge standard conjecture). For all $k$, the pairing $\langle,\rangle_{P^{k}(X)}$ is positive definite.

Remark 2.7. Usually, Conjecture 2.6 is stated for all projective smooth varieties $X$ without Assumption 2.1$](H d g(X)$ in [Kl]). It is a part of Grothendieck's standard conjectures, and known to hold in the following cases.

(1) If $F=\mathbb{C}$, it is a consequence of the existence of polarized Hodge structures on singular cohomology (We $)$. The general characteristic 0 case follows by Lefschetz principle. 
(2) If $X$ is of dimension $\leq 2$, it is known as the Hodge index theorem ([K], 5, $\mathrm{Fu}$, Example 15.2.4). As a corollary, we can prove the case $k \leq 1$ by taking hyperplane sections.

Remark 2.8. Note that Conjecture 2.6 is stated for a pair $(X, L)$. It seems that Conjecture 2.6 for $(X, L)$ does not automatically imply Conjecture 2.6 for $\left(X, L^{\prime}\right)$ with another choice of $L^{\prime}$ (see also Corollary 2.12 below).

Example 2.9. For an ample $\mathbb{R}$-divisor $L$ on $\mathbb{P}^{n}$, it is clear that Conjecture 2.4 holds for $\left(\mathbb{P}^{n}, L\right)$. The only nontrivial primitive cohomology is $P^{0}\left(\mathbb{P}^{n}\right)=$ $H^{0}\left(\mathbb{P}^{n}\right)=\mathbb{R}$ and the pairing $\langle,\rangle_{P^{0}\left(\mathbb{P}^{n}\right)}$ is

$$
P^{0}\left(\mathbb{P}^{n}\right) \times P^{0}\left(\mathbb{P}^{n}\right)=\mathbb{R} \times \mathbb{R} \ni(x, y) \mapsto L^{n} \cdot x y \in \mathbb{R}=H^{2 n}\left(\mathbb{P}^{n}\right) .
$$

Since $L^{n}$ is positive, this pairing is positive definite. Hence Conjecture 2.6 holds for $\left(\mathbb{P}^{n}, L\right)$ for all ample $\mathbb{R}$-divisors $L$ on $\mathbb{P}^{n}$.

2.4. Signatures. Let notation be as in 2.3. Firstly, we recall the definition of signatures. Let $V$ be a finite dimensional $\mathbb{R}$-vector space with a bilinear symmetric perfect pairing $\langle$,$\rangle . Take a basis \left\{e_{1}, \ldots, e_{r}\right\}$ of $V$ and consider the matrix $M=$ $\left(m_{i j}\right)$ defined by $m_{i j}=\left\langle e_{i}, e_{j}\right\rangle$. By Sylvester's law of inertia, all eigenvalues of $M$ are in $\mathbb{R}$, and the number of positive eigenvalues minus the number of negative eigenvalues is independent of the choice of $\left\{e_{1}, \ldots, e_{r}\right\}$. We denote this number by $\operatorname{sign}(V,\langle\rangle$,$) and call it the signature of the pairing \langle$,$\rangle on V$.

Proposition 2.10. The Hodge standard conjecture (Conjecture 2.6) holds for $(X, L)$ if and only if

$$
\operatorname{sign}\left(H^{k}(X),\langle,\rangle_{H^{k}(X)}\right)=\sum_{i \geq 0}(-1)^{i} \operatorname{dim}_{\mathbb{R}} P^{k-2 i}(X) \quad \text { for all } k \text { with } 0 \leq k \leq n .
$$

Remark 2.11. By Remark 2.5, the right hand side of Proposition 2.10] does not depend on a choice of $L$.

Proof. By definition, Conjecture 2.6 is equivalent to the following equality:

$$
\operatorname{dim}_{\mathbb{R}} P^{k}(X)=\operatorname{sign}\left(P^{k}(X),\langle,\rangle_{P^{k}(X)}\right) \quad \text { for all } k \text { with } 0 \leq k \leq n .
$$

By (2.2), we see that, for all $k$ with $0 \leq k \leq n$,

$$
\operatorname{sign}\left(H^{k}(X),\langle,\rangle_{H^{k}(X)}\right)=\sum_{i \geq 0}(-1)^{i} \operatorname{sign}\left(P^{k-2 i}(X),\langle,\rangle_{P^{k-2 i}(X)}\right) .
$$

Hence (2.3) implies the conditions in Proposition 2.10.

Conversely, assume the conditions in Proposition 2.10, We shall prove (2.3) by induction on $k$. The case $k=0$ is trivial, since $P^{0}(X) \cong \mathbb{R}$ and $L^{n}$ is positive. If 
(2.3) is proved for $k<k_{0}$, the condition of Proposition 2.10 for $k=k_{0}$ implies

$$
\begin{aligned}
\operatorname{sign} & \left(H^{k_{0}}(X),\langle,\rangle_{H^{k_{0}}(X)}\right) \\
& =\sum_{i \geq 0}(-1)^{i} \operatorname{dim}_{\mathbb{R}} P^{k_{0}-2 i}(X) \\
& =\operatorname{dim}_{\mathbb{R}} P^{k_{0}}(X)+\sum_{i \geq 1}(-1)^{i} \operatorname{sign}\left(P^{k_{0}-2 i}(X),\langle,\rangle_{P^{k_{0}-2 i}(X)}\right) .
\end{aligned}
$$

By (2.2), we have $\operatorname{dim}_{\mathbb{R}} P^{k_{0}}(X)=\operatorname{sign}\left(P^{k_{0}}(X),\langle,\rangle_{P^{k_{0}(X)}}\right)$.

Since the signature is locally constant on the set of non-degenerate quadratic forms over $\mathbb{R}$, we have the following implications about the Hodge standard conjecture (Conjecture 2.6) for a variety with different choices of ample $\mathbb{R}$-divisors on it.

Corollary 2.12. (1) Let $L, L^{\prime}$ be ample $\mathbb{R}$-divisors on $X$. If the hard Lefschetz conjecture (Conjecture 2.4) holds for $\left(X, t L+(1-t) L^{\prime}\right)$ for all $t \in \mathbb{R}$ with $0 \leq t \leq 1$, then the Hodge standard conjecture (Conjecture [2.6) for $(X, L),\left(X, L^{\prime}\right)$ are equivalent to each other.

(2) In particular, if Conjecture 2.4 holds for $(X, L)$ for all ample $\mathbb{R}$-divisors $L$ on $X$, then Conjecture 2.6 for $(X, L)$ for all ample $\mathbb{R}$-divisors $L$ on $X$ are equivalent to each other.

2.5. Products of varieties. As in $\$ 2.1$ let $X$ (resp. $Y$ ) be a projective smooth variety over $F$ satisfying Assumption 2.1 and $L_{X}$ (resp. $L_{Y}$ ) an ample $\mathbb{R}$-divisor on $X$ (resp. $Y$ ). Then, by the Künneth formula, we have an isomorphism:

$$
H^{k}(X \times Y) \cong \bigoplus_{i=0}^{k} H^{i}(X) \otimes_{\mathbb{R}} H^{k-i}(Y)
$$

Hence $X \times Y$ also satisfies Assumption 2.1. The condition that $L_{X}, L_{Y}$ are ample is equivalent to the condition that $\operatorname{pr}_{1}^{*} \mathscr{O}\left(L_{X}\right)+\operatorname{pr}_{2}^{*} \mathscr{O}\left(L_{Y}\right)$ is ample, where $\operatorname{pr}_{1}: X \times Y \rightarrow X$ and $\mathrm{pr}_{2}: X \times Y \rightarrow Y$ are projections (Hart1], I, Hart2, II).

Proposition 2.13. (1) If the hard Lefschetz conjecture (Conjecture 2.4) holds for $\left(X, L_{X}\right),\left(Y, L_{Y}\right)$, then it also holds for $\left(X \times Y, \operatorname{pr}_{1}^{*} \mathscr{O}\left(L_{X}\right)+\operatorname{pr}_{2}^{*} \mathscr{O}\left(L_{Y}\right)\right)$.

(2) Moreover, if the Hodge standard conjecture (Conjecture 2.6) holds for $\left(X, L_{X}\right),\left(Y, L_{Y}\right)$, then it also holds for $\left(X \times Y, \operatorname{pr}_{1}^{*} \mathscr{O}\left(L_{X}\right)+\operatorname{pr}_{2}^{*} \mathscr{O}\left(L_{Y}\right)\right)$.

Proof. Here we give a proof by using the representation theory of the Lie algebra $\mathfrak{s l}(2)$ (for details, De3, 1.6, Bourbaki Lie, VIII, §1). It is a consequence of Conjecture 2.4 that we can construct $\mathfrak{s l}(2)$-representations on $H^{*}(X), H^{*}(Y)$ by using the Lefschetz operator $L_{X}, L_{Y}$. Then, the tensor product $H^{*}(X) \otimes_{\mathbb{R}} H^{*}(Y)$ has a natural $\mathfrak{s l}(2)$-action which corresponds to $\operatorname{pr}_{1}^{*} \mathscr{O}\left(L_{X}\right)+\operatorname{pr}_{2}^{*} \mathscr{O}\left(L_{Y}\right)$. This proves the first assertion. The second assertion follows from a basic property of certain pairings defined on $\mathfrak{s l}(2)$-representations over $\mathbb{R}$ (Bourbaki Lie, VIII, $\S 1)$. 
Corollary 2.14. If Conjecture 2.4 (resp. Conjecture [2.6) holds for $\left(X, L_{X}\right)$, $\left(Y, L_{Y}\right)$ for all ample $\mathbb{R}$-divisors $L_{X}, L_{Y}$ on $X, Y$, then it also holds for $(X \times$ $\left.Y, L_{X \times Y}\right)$ for all ample $\mathbb{R}$-divisors $L_{X \times Y}$ on $X \times Y$.

Proof. We may assume that $X, Y$ are connected. Then we have $H^{2}(X \times Y) \cong$ $H^{2}(X) \oplus H^{2}(Y)$, and all ample $\mathbb{R}$-divisors on $X \times Y$ are necessarily of the form $\operatorname{pr}_{1}^{*} \mathscr{O}\left(L_{X}\right)+\operatorname{pr}_{2}^{*} \mathscr{O}\left(L_{Y}\right)$ in $H^{2}(X \times Y)$. Hence the assertion follows from Proposition 2.13

\section{Cohomology of VARIETiEs obtained By BLOWING-UPS}

In this section, we study the Hodge standard conjecture (Conjecture 2.6) for varieties obtained by blowing-ups. After recalling basic facts about blowing-ups, we show that, under certain assumptions, Conjecture 2.6 holds for a blow-up for certain choice of an ample $\mathbb{R}$-divisor (for details of the computation, see [SGA5], VII, Hart2, II, Fu], 6.7). We use the same notation as in $\$ 2.1$.

3.1. Setup. Let $X$ be a projective smooth irreducible variety over $F$ of dimension $n$, and $Y_{1}, \ldots, Y_{r} \subset X$ mutually disjoint smooth closed irreducible subvarieties of codimension $d \geq 2$. Let $i: Y=\coprod_{k=1}^{r} Y_{k} \hookrightarrow X$ be the closed immersion. Let $f: X^{\prime} \rightarrow X$ be the blow-up of $X$ along $Y, Y^{\prime} \subset X^{\prime}$ the strict transform of $Y$, and $j: Y^{\prime} \hookrightarrow X^{\prime}, g: Y^{\prime} \rightarrow Y$ natural morphisms. Assume that $X, Y_{1}, \ldots, Y_{r}$ satisfy Assumption [2.1 in $\$ 2.1$. Then, it is easy to see that $X^{\prime}, Y^{\prime}$ also satisfy Assumption 2.1] in $\$ 2.1$. This can be checked from the computations in 3.2 of the cohomology of $X^{\prime}$ and $Y^{\prime}$.

We have the following cartesian diagram:

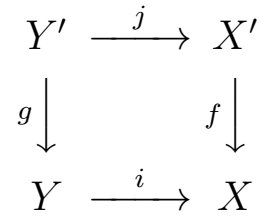

Since $Y$ is a disjoint union of mutually disjoint smooth closed irreducible subvarieties of codimension $d, g: Y^{\prime} \rightarrow Y$ has a structure of $\mathbb{P}^{d-1}$-bundle. More precisely, $Y^{\prime}=\mathbb{P}\left(\check{N}_{Y / X}\right)$, where $\check{N}_{Y / X}$ is the conormal bundle of $Y$ in $X$. Let

$$
\xi=c_{1}\left(\mathscr{O}_{Y^{\prime}}(1)\right) \in H^{2}\left(Y^{\prime}\right)
$$

be the first Chern class of $\mathscr{O}_{Y^{\prime}}(1)$ of the $\mathbb{P}^{d-1}$-bundle $g: Y^{\prime} \rightarrow Y$. The restriction of the line bundle $\mathscr{O}_{X^{\prime}}\left(Y^{\prime}\right)$ to $Y^{\prime}$ itself is isomorphic to $\mathscr{O}_{Y^{\prime}}(-1)$.

We define a vector bundle $F$ of rank $d-1$ on $Y^{\prime}$ by the kernel of a natural map $g^{*}\left(\check{N}_{Y / X}\right) \rightarrow \mathscr{O}_{Y^{\prime}}(1)$. Then we have the following exact sequence of vector bundles on $Y^{\prime}$ :

$$
0 \longrightarrow F \longrightarrow g^{*}\left(\check{N}_{Y / X}\right) \longrightarrow \mathscr{O}_{Y^{\prime}}(1) \longrightarrow 0 .
$$


3.2. Cohomology of $X^{\prime}$ and $Y^{\prime}$. For all $k$, we have the following exact sequence of cohomology groups ([SGA5], VII, 8.5):

$$
0 \longrightarrow H^{k-2 d}(Y) \stackrel{\lambda}{\longrightarrow} H^{k-2}\left(Y^{\prime}\right) \oplus H^{k}(X) \stackrel{\mu}{\longrightarrow} H^{k}\left(X^{\prime}\right) \longrightarrow 0,
$$

where $\lambda$ and $\mu$ are defined as follows:

$$
\lambda(y)=\left(g^{*}(y) \cup c_{d-1}(\check{F}),-i_{*}(y)\right), \quad \mu(y, x)=j_{*}(y)+f^{*}(x) .
$$

Note that $\check{F}$ is the dual vector bundle of $F$ and $c_{d-1}(\check{F}) \in H^{2 d-2}\left(Y^{\prime}\right)$ is the $(d-1)$-th Chern class of $\check{F}$. Define $\lambda^{\prime}: H^{k-2}\left(Y^{\prime}\right) \oplus H^{k}(X) \rightarrow H^{k-2 d}(Y)$ by $\lambda^{\prime}(y, x)=g_{*}(y)$, then we have

$$
\lambda^{\prime} \circ \lambda(y)=g_{*}\left(g^{*}(y) \cup c_{d-1}(\check{F})\right)=y \cup g_{*}\left(c_{d-1}(\check{F})\right)=y
$$

since $g_{*}\left(c_{d-1}(\check{F})\right)=(-1)^{d-1} g_{*}\left(c_{d-1}(F)\right)=g_{*}\left(\xi^{d-1}\right)=1$ ([SGA5], VII, 8.4.2). Therefore, we have the following isomorphism of cohomology groups:

$$
H^{k}\left(X^{\prime}\right) \stackrel{\mu}{\cong} \operatorname{Ker}\left(g_{*}: H^{k-2}\left(Y^{\prime}\right) \rightarrow H^{k-2 d}(Y)\right) \oplus H^{k}(X)
$$

We shall compute the right hand side explicitly. For an integer $m$ with $0 \leq$ $m \leq d-1$, the map $H^{k-2 m}(Y) \rightarrow H^{k}\left(Y^{\prime}\right), y \mapsto g^{*}(y) \cup \xi^{m}$ is injective because

$$
g_{*}\left(\left(g^{*}(y) \cup \xi^{m}\right) \cup \xi^{d-1-m}\right)=y \cup g_{*}\left(\xi^{d-1}\right)=y .
$$

Let $H^{k-2 m}(Y) \xi^{m} \subset H^{k}\left(Y^{\prime}\right)$ be the image of this map. For simplicity, we sometimes write $y \xi^{m}$ instead of $g^{*}(y) \cup \xi^{m}$. Note that $g_{*}: H^{k}\left(Y^{\prime}\right) \rightarrow H^{k-2(d-1)}(Y)$ satisfies

$$
g_{*}\left(a \xi^{m}\right)=a \cup g_{*}\left(\xi^{m}\right)= \begin{cases}a & \text { if } m=d-1 \\ 0 & \text { otherwise. }\end{cases}
$$

On the other hand, we have an isomorphism ([SGA5], VII, 2.2.6):

$$
\begin{aligned}
H^{k}\left(Y^{\prime}\right) & \cong \bigoplus_{m=0}^{d-1} H^{k-2 m}(Y) \xi^{m} \\
& =H^{k}(Y) \oplus H^{k-2}(Y) \xi \oplus \cdots \oplus H^{k-2(d-1)}(Y) \xi^{d-1}
\end{aligned}
$$

Therefore, by (3.2), we have the following explicit description of the cohomology groups of $X^{\prime}$ :

$$
H^{k}\left(X^{\prime}\right) \cong H^{k-2}(Y) \oplus H^{k-4}(Y) \xi \oplus \cdots \oplus H^{k-2-2(d-2)}(Y) \xi^{d-2} \oplus H^{k}(X) .
$$

The following property of the cohomology of $X^{\prime}$ will be used later.

Proposition 3.1. Let $x^{\prime} \in H^{k}\left(X^{\prime}\right)$ be a cohomology class on $X^{\prime}$ whose restriction to $Y^{\prime}$ is zero. Then $x^{\prime}$ is of the form $x^{\prime}=f^{*}(x)$ for a unique $x \in H^{k}(X)$.

Proof. The uniqueness is clear because $f_{*}\left(x^{\prime}\right)=f_{*}\left(f^{*}(x)\right)=x$. For the existence, we write $x^{\prime}$ of the form $x^{\prime}=x_{0}+x_{1} \xi+\cdots+x_{d-2} \xi^{d-2}+x$ via (3.3). This means that there is a relation

$$
x^{\prime}=j_{*}\left(x_{0}+x_{1} \xi+\cdots+x_{d-2} \xi^{d-2}\right)+f^{*}(x) .
$$


It is enough to show $x_{0}=x_{1}=\cdots=x_{d-2}=0$. Since the restriction of $\mathscr{O}_{X^{\prime}}\left(Y^{\prime}\right)$ to $Y^{\prime}$ is isomorphic $\mathscr{O}_{Y^{\prime}}(-1)$, we have $j^{*}\left(j_{*}\left(1_{Y^{\prime}}\right)\right)=-\xi$. Therefore,

$$
\begin{aligned}
j^{*}\left(x^{\prime}\right) & =j^{*} j_{*}\left(x_{0}+x_{1} \xi+\cdots+x_{d-2} \xi^{d-2}\right)+j^{*} f^{*}(x) \\
& =-x_{0} \xi-x_{1} \xi^{2}-\cdots-x_{d-2} \xi^{d-1}+g^{*} i^{*}(x) .
\end{aligned}
$$

On the other hand, $j^{*}\left(x^{\prime}\right)=0$ by assumption. Since $H^{*}\left(Y^{\prime}\right)$ is a free $H^{*}(Y)$ module with basis $1, \xi, \ldots, \xi^{d-1}$, we have $x_{0}=x_{1}=\cdots=x_{d-2}=i^{*}(x)=0$.

3.3. Multiplicative structure of $H^{*}\left(X^{\prime}\right)$. The multiplicative structure of $H^{*}\left(X^{\prime}\right)$ can be computed as follows. Define $\nabla^{k}\left(X^{\prime}\right)$ by

$$
\nabla^{k}\left(X^{\prime}\right)=H^{k-2}\left(Y^{\prime}\right) \oplus H^{k}(X)
$$

and $\cup^{\prime}: \nabla^{k}\left(X^{\prime}\right) \times \nabla^{m}\left(X^{\prime}\right) \rightarrow \nabla^{k+m}\left(X^{\prime}\right)$ by

$$
\left(y_{1}^{\prime}, x_{1}\right) \cup^{\prime}\left(y_{2}^{\prime}, x_{2}\right)=\left(-y_{1}^{\prime} \cup y_{2}^{\prime} \cup \xi+y_{1}^{\prime} \cup g^{*} i^{*}\left(x_{2}\right)+(-1)^{k m} y_{2}^{\prime} \cup g^{*} i^{*}\left(x_{1}\right), x_{1} \cup x_{2}\right) \text {. }
$$

Then the following diagram is commutative ([SGA5], VII, 8.6.3):

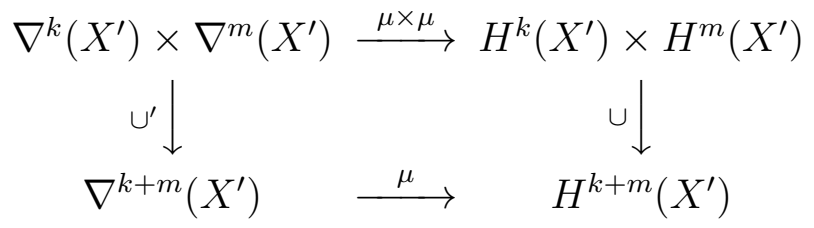

where $\cup$ denotes the cup product on $H^{*}\left(X^{\prime}\right)$. Since the horizontal maps $\mu, \mu \times \mu$ are surjective, we can compute the multiplicative structure of $H^{*}\left(X^{\prime}\right)$ in terms of the multiplicative structure of $\nabla^{*}\left(X^{\prime}\right):=\bigoplus_{k} \nabla^{k}\left(X^{\prime}\right)$.

Recall that the multiplicative structure of $H^{*}\left(Y^{\prime}\right)$ is computed by Chern classes ([SGA5], VII, 3). Namely, we have the following isomorphism of $H^{*}(Y)$-algebras

$$
H^{*}\left(Y^{\prime}\right) \cong H^{*}(Y)[T] /\left(T^{d}+c_{1}\left(\check{N}_{Y / X}\right) T^{d-1}+c_{2}\left(\check{N}_{Y / X}\right) T^{d-2}+\cdots+c_{d}\left(\check{N}_{Y / X}\right)\right) .
$$

which sends $\xi$ to $T$.

There is another relation on $H^{*}\left(X^{\prime}\right)$ coming from the exact sequence (3.1). Namely, the image of $1_{Y} \in H^{0}(Y)$ by $\lambda$,

$$
\lambda\left(1_{Y}\right)=\left(c_{d-1}(\check{F}),-i_{*}\left(1_{Y}\right)\right) \in H^{2 d-2}\left(Y^{\prime}\right) \oplus H^{2 d}(X),
$$

maps to 0 in $H^{2 d}\left(X^{\prime}\right)$. By definition, $i_{*}\left(1_{Y}\right) \in H^{2 d}(X)$ is nothing but the cohomology class of $Y$. We denote it by $[Y]$. Since the coefficient of $\xi^{d-1}$ in the expression of $c_{d-1}(\check{F})$ is 1 ([SGA5], VII, 8.4.2), we can replace $\xi^{d-1}$ by $\left(\xi^{d-1}-c_{d-1}(\check{F}),[Y]\right)$.

In conclusion, we can compute the cup product on $H^{*}\left(X^{\prime}\right)$ in terms of the cohomology groups $H^{*}(X), H^{*}(Y)$, the Chern classes of $\check{N}_{Y / X}$, and the cohomology class $[Y] \in H^{2 d}(X)$. 


\subsection{Ample divisors on $X^{\prime}$.}

Proposition 3.2. Let notation be as in 3.1 . Let $L$ be an ample $\mathbb{R}$-divisor on $X$. There exists $\alpha \in \mathbb{R}_{>0}$ such that, for all $\varepsilon \in \mathbb{R}$, the $\mathbb{R}$-divisor $L^{\prime}$ on $X^{\prime}$ of the form

$$
L^{\prime}=f^{*} \mathscr{O}(L)-\varepsilon Y^{\prime}
$$

is ample if and only if $0<\varepsilon<\alpha$.

Proof. We use the following basic facts. Although some results quoted below are only stated for usual ample divisors in the literature (for example, see Hart1, Hart2 ), it is easy to prove them for ample $\mathbb{R}$-divisors.

(1) Take an effective curve $C$ on $X^{\prime}$ which passes through a point of $Y^{\prime}$ and intersects with $Y^{\prime}$ transversally. By the Nakai-Moishezon criterion of ampleness ([Hart1], I, §5), we have $\left(f^{*} \mathscr{O}(L)-\varepsilon Y^{\prime}\right) \cdot C>0$. Since $Y^{\prime} \cdot C>0$, $\varepsilon$ can not be arbitrary large.

(2) If $f^{*} \mathscr{O}(L)-\varepsilon Y^{\prime}$ is ample, then $\varepsilon>0$. To see this, take an effective curve $C$ contained in a fiber of $g: Y^{\prime} \rightarrow Y$. Since $f^{*} \mathscr{O}(L) \cdot C=L \cdot f_{*} C=0$ and $Y^{\prime} \cdot C<0$, we have $\varepsilon>0$ by the Nakai-Moishezon criterion of ampleness.

(3) We see that $f^{*} \mathscr{O}(L)-\varepsilon Y^{\prime}$ is ample for sufficiently small $\varepsilon \in \mathbb{R}_{>0}$, because the restriction of $\mathscr{O}_{X^{\prime}}\left(-Y^{\prime}\right)$ to $Y^{\prime}$ is isomorphic to $\mathscr{O}_{Y^{\prime}}(1)$ ([Hart2], II, $7.10)$.

(4) The set $I=\left\{\varepsilon \in \mathbb{R} \mid f^{*} \mathscr{O}(L)-\varepsilon Y^{\prime}\right.$ is ample $\}$ is an interval, because if $D, D^{\prime}$ are ample $\mathbb{R}$-divisors, then $t D+(1-t) D^{\prime}$ are also ample for all $t \in \mathbb{R}$ with $0 \leq t \leq 1$.

(5) Moreover, $I$ is an open interval because, for a $\mathbb{R}$-divisor $D$ and an ample $\mathbb{R}$-divisor $D^{\prime}, t D+(1-t) D^{\prime}$ is ample for sufficiently small $t \in \mathbb{R}_{>0}$.

By combining above facts, we conclude that $I=\{\varepsilon \in \mathbb{R} \mid 0<\varepsilon<\alpha\}$ for some $\alpha \in \mathbb{R}_{>0}$.

Remark 3.3. Since all $\mathbb{R}$-divisors $L^{\prime}$ on $X^{\prime}$ can be written as $L^{\prime}=f^{*} f_{*} \mathscr{O}\left(L^{\prime}\right)-\varepsilon Y^{\prime}$ for some $\varepsilon \in \mathbb{R}$, Proposition 3.2 determines the set of all ample $\mathbb{R}$-divisors on $X^{\prime}$ in some sense. However, in practice, it seems difficult to compute the upper bound $\alpha$ in Proposition 3.2 explicitly.

\subsection{Hodge standard conjecture for $X^{\prime}$.}

Proposition 3.4. Let notation be as in 3.1 (Recall that $X, Y, X^{\prime}, Y^{\prime}$ satisfy Assumption 2.1 in (2.1). Let $L$ be an ample $\mathbb{R}$-divisor on $X$. Assume that the hard Lefschetz conjecture (Conjecture 2.4) and the Hodge standard conjecture (Conjecture [2.6) hold for $(X, L)$ and $\left(Y,\left.\mathscr{O}(L)\right|_{Y}\right)$, where $\left.\mathscr{O}(L)\right|_{Y}$ denotes the restriction of $\mathscr{O}(L)$ to $Y$ which is also ample. Then, there exists $\beta \in \mathbb{R}_{>0}$ such that, for all $\varepsilon \in \mathbb{R}$ with $0<\varepsilon<\beta$, the $\mathbb{R}$-divisor $L^{\prime}$ on $X^{\prime}$ of the form

$$
L^{\prime}=f^{*} \mathscr{O}(L)-\varepsilon Y^{\prime}
$$

is an ample $\mathbb{R}$-divisor on $X^{\prime}$ for which Conjecture 2.4 and Conjecture 2.6 hold. 
Proof. First of all, by the isomorphism (3.3), we observe that $H^{*}\left(X^{\prime}\right)$ is isomorphic to $H^{*}\left(Y \times \mathbb{P}^{d-2}\right) \oplus H^{*}(X)$ as an $\mathbb{R}$-vector space, although the multiplicative structures are different. Nevertheless, if we choose appropriate bases of $H^{*}\left(X^{\prime}\right)$ and $H^{*}\left(Y \times \mathbb{P}^{d-2}\right) \oplus H^{*}(X)$, and take the limit $\varepsilon \rightarrow 0$, the matrices representing the action of the Lefschetz operator and the cup product pairings on $H^{*}\left(X^{\prime}\right)$ converge to those on $H^{*}\left(Y \times \mathbb{P}^{d-2}\right) \oplus H^{*}(X)$.

Let us make the above observation precise. Let $\left\{x_{\gamma}\right\}$ (resp. $\left\{y_{\delta}\right\}$ ) be the basis of $H^{*}(X)$ (resp. $H^{*}(Y)$ ) which is independent of $\varepsilon$. For $\varepsilon \in \mathbb{R}_{>0}$, we see that $\left\{\varepsilon^{-(d-2) / 2+k} y_{\delta} \xi^{k}\right\}$ is a $\varepsilon$-dependent basis of $H^{*}(Y) \xi^{k}$ for $0 \leq k \leq d-2$. By (3.3), we have the following isomorphism:

$$
H^{*}\left(X^{\prime}\right) \cong H^{*}(Y) \oplus H^{*}(Y) \xi \oplus \cdots \oplus H^{*}(Y) \xi^{d-2} \oplus H^{*}(X) .
$$

such that the following set

$$
\left\{\varepsilon^{-(d-2) / 2} y_{\delta}\right\} \cup\left\{\varepsilon^{-(d-2) / 2+1} y_{\delta} \xi\right\} \cup \cdots \cup\left\{\varepsilon^{-(d-2) / 2+(d-2)} y_{\delta} \xi^{d-2}\right\} \cup\left\{x_{\gamma}\right\}
$$

is a basis of $H^{*}\left(X^{\prime}\right)$. By using the basis (3.4), we have an isomorphism

$$
H^{k}\left(X^{\prime}\right) \cong H^{k-2}\left(Y \times \mathbb{P}^{d-2}\right) \oplus H^{k}(X)
$$

as an $\mathbb{R}$-vector space which sends $\varepsilon^{-(d-2) / 2+m} \xi^{m}$ to $c_{1}\left(\mathscr{O}_{\mathbb{P}^{d-2}}(1)\right)^{m}$. The reason we multiply $\xi^{k}$ by $\varepsilon^{-(d-2) / 2+k}$ in (3.4) is as follows. If we take the cup product of two elements in (3.4), the coefficient of the top degree term $\xi^{d-2}$ is a constant function in $\varepsilon$. If $\xi^{k}$ for $k>d-2$ appears in the cup product, we can replace $\xi^{d-1}$ by $\left(\xi^{d-1}-c_{d-1}(\check{F}),[Y]\right)$ in $H^{*}\left(X^{\prime}\right)$ (see $\left.\$ 3.3\right)$. However, since the order of $\varepsilon$ in the coefficient of $\xi^{d-1}$ is one, this term vanishes if we take the limit $\varepsilon \rightarrow 0$. Therefore, by using the basis (3.4), we can consider the asymptotic behavior of the Lefschetz operator and the cup product pairings on $H^{*}\left(X^{\prime}\right)$ as $\varepsilon \rightarrow 0$.

We put $\widetilde{H}^{k}\left(X^{\prime}\right):=H^{k-2}\left(Y \times \mathbb{P}^{d-2}\right) \oplus H^{k}(X)$ and define the map

$$
\widetilde{L}: \widetilde{H}^{k}\left(X^{\prime}\right) \longrightarrow \widetilde{H}^{k+2}\left(X^{\prime}\right)
$$

as follows. Let $\mathrm{pr}_{1}: Y \times \mathbb{P}^{d-2} \rightarrow Y, \mathrm{pr}_{2}: Y \times \mathbb{P}^{d-2} \rightarrow \mathbb{P}^{d-2}$ be projections. Then

$$
L_{Y \times \mathbb{P}^{d-2}}=\operatorname{pr}_{1}^{*}\left(\left.\mathscr{O}(L)\right|_{Y}\right)+\operatorname{pr}_{2}^{*} \mathscr{O}_{\mathbb{P}^{d-2}}(1)
$$

is an ample $\mathbb{R}$-divisor on $Y \times \mathbb{P}^{d-2}$. We define the map $\widetilde{L}$ as a direct sum of the Lefschetz operators $L_{Y \times \mathbb{P}^{d-2}}$ and $L$. By Example 2.9. Proposition 2.13, the hard Lefschetz conjecture (Conjecture 2.4) and the Hodge standard conjecture (Conjecture 2.6) hold for $\left(Y \times \mathbb{P}^{d-2}, L_{Y \times \mathbb{P}^{d-2}}\right)$. Therefore, we see that $\widetilde{L}^{k}$ induces the following isomorphism:

$$
\widetilde{L}^{k}: \widetilde{H}^{n-k}\left(X^{\prime}\right) \stackrel{\cong}{\longrightarrow} \widetilde{H}^{n+k}\left(X^{\prime}\right) .
$$

Namely, $\widetilde{L}$ satisfies an analogue of the hard Lefschetz conjecture (Conjecture 2.4). Moreover, an analogue of the Hodge standard conjecture (Conjecture 2.6) holds for $\widetilde{L}$ in the following sense. Let $\widetilde{P}^{k}\left(X^{\prime}\right)$ be the kernel of $\widetilde{L}^{n-k+1}$ on $\widetilde{H}^{k}\left(X^{\prime}\right)$, 
which is isomorphic to $P^{k-2}\left(Y \times \mathbb{P}^{d-2}\right) \oplus P^{k}(X)$. Then the following pairing on $\widetilde{P}^{k}\left(X^{\prime}\right)$

$$
\widetilde{P}^{k}\left(X^{\prime}\right) \times \widetilde{P}^{k}\left(X^{\prime}\right) \longrightarrow \widetilde{H}^{2 n}\left(X^{\prime}\right)=\mathbb{R}, \quad(a, b) \mapsto(-1)^{k / 2} \widetilde{L}^{n-k} a \widetilde{\cup} b
$$

is positive definite, where $\widetilde{\cup}$ is a difference of the cup products on $H^{*}\left(Y \times \mathbb{P}^{d-2}\right)$ and $H^{*}(X)$ defined as follows:

$$
\widetilde{\cup}: \widetilde{H}^{*}\left(X^{\prime}\right) \times \widetilde{H}^{*}\left(X^{\prime}\right) \ni\left(\left(y_{0}, x_{0}\right),\left(y_{1}, x_{1}\right)\right) \mapsto-y_{0} \cup y_{1}+x_{0} \cup x_{1} .
$$

Note that the minus sign is inevitable for the Hodge standard conjecture (Conjecture (2.6) because, for $(y, x) \in \widetilde{P}^{k}\left(X^{\prime}\right)$, the degree of $y$ is smaller than the degree of $x$ by 2 .

Therefore, to prove Proposition [3.4 it is enough to prove the following lemma.

Lemma 3.5. If we take the limit $\varepsilon \rightarrow 0$, the matrices representing the action of $L^{\prime}=f^{*} \mathscr{O}(L)-\varepsilon Y^{\prime}$ with respect to the basis (3.4) converge to the matrices representing $\widetilde{L}$ via (3.5). Similarly, for all $k$, the matrices representing the cup product pairing between $H^{k}\left(X^{\prime}\right)$ and $H^{2 n-k}\left(X^{\prime}\right)$ converge to the matrices representing the pairing $\widetilde{\cup}$ between $\widetilde{H}^{k}\left(X^{\prime}\right)$ and $\widetilde{H}^{2 n-k}\left(X^{\prime}\right)$.

We shall compute the action of $L^{\prime}=f^{*} \mathscr{O}(L)-\varepsilon Y^{\prime}$. Since $\left[Y^{\prime}\right]=j_{*}\left(1_{Y^{\prime}}\right) \in$ $H^{2}\left(X^{\prime}\right)$, we compute the cup product

$$
\left(f^{*} \mathscr{O}(L)-\varepsilon j_{*}\left(1_{Y^{\prime}}\right)\right) \cup\left(f^{*}(x)+j_{*}(y)\right)
$$

for $x \in H^{k}(X), y \in H^{k-2}\left(Y^{\prime}\right)$ with $g_{*}(y)=0$ (see (3.2) ). Since (3.7) is equal to

$$
f^{*} \mathscr{O}(L) \cup f^{*}(x)+f^{*} \mathscr{O}(L) \cup j_{*}(y)-\varepsilon j_{*}\left(1_{Y^{\prime}}\right) \cup f^{*}(x)-\varepsilon j_{*}\left(1_{Y^{\prime}}\right) \cup j_{*}(y),
$$

we consider these four terms separately. Firstly, since the pullback preserves the cup product, we have $f^{*} \mathscr{O}(L) \cup f^{*}(x)=f^{*}(\mathscr{O}(L) \cup x)=f^{*}(L \cup x)$. Secondly, we have $f^{*} \mathscr{O}(L) \cup j_{*}(y)=j_{*}\left(j^{*} f^{*} \mathscr{O}(L) \cup y\right)=j_{*}\left(g^{*} i^{*} \mathscr{O}(L) \cup y\right)=j_{*}\left(g^{*}\left(\left.\mathscr{O}(L)\right|_{Y}\right) y\right)$ because $f \circ j=i \circ g$. Similarly, we have $-\varepsilon j_{*}\left(1_{Y^{\prime}}\right) \cup f^{*}(x)=-\varepsilon j_{*}\left(1_{Y^{\prime}} \cup j^{*} f^{*}(x)\right)=$ $-\varepsilon j_{*}\left(g^{*}\left(\left.x\right|_{Y}\right)\right)$. Finally, by the self-intersection formula $j^{*} j_{*}\left(1_{Y^{\prime}}\right)=-\xi$, we have $-\varepsilon j_{*}\left(1_{Y^{\prime}}\right) \cup j_{*}(y)=-\varepsilon j_{*}\left(j^{*} j_{*}\left(1_{Y^{\prime}}\right) \cup y\right)=j_{*}(\varepsilon \xi \cup y)$. Therefore, we conclude that (3.7) is equal to

$$
f^{*}(L \cup x)+j_{*}\left(\left(g^{*}\left(\left.\mathscr{O}(L)\right|_{Y}\right)+\varepsilon \xi\right) \cup y\right)-\varepsilon j_{*}\left(g^{*}\left(\left.x\right|_{Y}\right)\right) .
$$

It is clear that the first term $f^{*}(L \cup x)$ corresponds to the action of $L$ on $H^{*}(X)$ via (3.5). The second term $j_{*}\left(\left(g^{*}\left(\left.\mathscr{O}(L)\right|_{Y}\right)+\varepsilon \xi\right) \cup y\right)$ almost corresponds to the action of $L_{Y \times \mathbb{P}^{d-2}}$ on $H^{*}\left(Y \times \mathbb{P}^{d-2}\right)$ via (3.5). The only difference is that $\xi^{d-1}$ may appear in the cup product $\left(g^{*}\left(\left.\mathscr{O}(L)\right|_{Y}\right)+\varepsilon \xi\right) \cup y$. Since the order of $\varepsilon$ in the coefficient of $\xi^{d-1}$ is higher than others, if we replace $\xi^{d-1}$ by $\left(\xi^{d-1}-c_{d-1}(\check{F}),[Y]\right)$, this contribution vanishes if we take the limit $\varepsilon \rightarrow 0$. The contribution from the third term $-\varepsilon j_{*}\left(g^{*}\left(\left.x\right|_{Y}\right)\right)$ vanishes if we take the limit $\varepsilon \rightarrow 0$. Hence the first assertion of Lemma 3.5 is proved. 
Finally, we shall compute the cup product pairing on $H^{*}\left(X^{\prime}\right)$. According to (3.2), we compute the cup product

$$
\left(f^{*}\left(x_{0}\right)+j_{*}\left(y_{0}\right)\right) \cup\left(f^{*}\left(x_{1}\right)+j_{*}\left(y_{1}\right)\right)
$$

for $x_{0} \in H^{k}(X), y_{0} \in H^{k-2}\left(Y^{\prime}\right), x_{1} \in H^{2 n-k}(X), y_{1} \in H^{2 n-k-2}\left(Y^{\prime}\right)$ with $g_{*}\left(y_{0}\right)=$ $0, g_{*}\left(y_{1}\right)=0$. Since (3.8) is in $H^{2 n}\left(X^{\prime}\right)=\mathbb{R}$, and $f_{*}: H^{2 n}\left(X^{\prime}\right) \rightarrow H^{2 n}(X)$ is an isomorphism, it is enough to compute

$$
\begin{aligned}
f_{*}\left(\left(f^{*}\left(x_{0}\right)+j_{*}\left(y_{0}\right)\right) \cup\left(f^{*}\left(x_{1}\right)+j_{*}\left(y_{1}\right)\right)\right) & =f_{*}\left(f^{*}\left(x_{0}\right) \cup f^{*}\left(x_{1}\right)\right) \\
& +f_{*}\left(f^{*}\left(x_{0}\right) \cup j_{*}\left(y_{1}\right)\right)+f_{*}\left(j_{*}\left(y_{0}\right) \cup f^{*}\left(x_{1}\right)\right)+f_{*}\left(j_{*}\left(y_{0}\right) \cup j_{*}\left(y_{1}\right)\right) .
\end{aligned}
$$

For the first term, we have $f_{*}\left(f^{*}\left(x_{0}\right) \cup f^{*}\left(x_{1}\right)\right)=f_{*}\left(f^{*}\left(x_{0} \cup x_{1}\right)\right)=x_{0} \cup x_{1}$ since the pullback preserves the cup product and $f_{*} \circ f^{*}=\mathrm{id}$. For the second term, we have $f_{*}\left(f^{*}\left(x_{0}\right) \cup j_{*}\left(y_{1}\right)\right)=x_{0} \cup f_{*}\left(j_{*}\left(y_{1}\right)\right)=x_{0} \cup i_{*}\left(g_{*}\left(y_{1}\right)\right)=0$ because $f \circ j=i \circ g$ and $g_{*}\left(y_{1}\right)=0$. Similarly, the third term $f_{*}\left(j_{*}\left(y_{0}\right) \cup f^{*}\left(x_{1}\right)\right)$ is zero because $g_{*}\left(y_{0}\right)=0$. For the last term, by the self-intersection formula $j^{*} j_{*}\left(y_{0}\right)=-\xi \cup y_{0}$, we have $f_{*}\left(j_{*}\left(y_{0}\right) \cup j_{*}\left(y_{1}\right)\right)=f_{*}\left(j_{*}\left(j^{*} j_{*}\left(y_{0}\right) \cup y_{1}\right)\right)=i_{*}\left(g_{*}\left(-\xi \cup y_{0} \cup y_{1}\right)\right)$. Since $g_{*}\left(\xi^{d-1}\right)=1$ and $g_{*}\left(\xi^{m}\right)=0$ for $0 \leq m \leq d-2$, if we take the limit $\varepsilon \rightarrow 0$, the matrix representing the pairing

$$
H^{k-2}\left(Y^{\prime}\right) \times H^{2 n-k-2}\left(Y^{\prime}\right) \ni\left(y_{0}, y_{1}\right) \mapsto i_{*}\left(g_{*}\left(-\xi \cup y_{0} \cup y_{1}\right)\right) \in H^{2 n}(X)=\mathbb{R}
$$

with respect to the basis (3.4) converges to the minus of the matrix representing the cup product pairing

$$
H^{k-2}\left(Y \times \mathbb{P}^{d-2}\right) \times H^{2 n-k-2}\left(Y \times \mathbb{P}^{d-2}\right) \longrightarrow H^{2 n-4}\left(Y \times \mathbb{P}^{d-2}\right)=\mathbb{R}
$$

via (3.5). Therefore, the second assertion of Lemma 3.5 is proved. Now the proof of Proposition 3.4 is complete.

Corollary 3.6. Let notation and assumptions be as in Proposition 3.4.

(1) If the hard Lefschetz conjecture (Conjecture 2.4) holds for $\left(X^{\prime}, L^{\prime}\right)$ for all ample $\mathbb{R}$-divisors $L^{\prime}$ on $X^{\prime}$ of the form $L^{\prime}=f^{*} \mathscr{O}(L)-\varepsilon Y^{\prime}\left(\varepsilon \in \mathbb{R}_{>0}\right)$, then the Hodge standard conjecture (Conjecture 2.6) holds for $\left(X^{\prime}, L^{\prime}\right)$ for all ample $\mathbb{R}$-divisors $L^{\prime}$ on $X^{\prime}$ of the form $L^{\prime}=f^{*} \mathscr{O}(L)-\varepsilon Y^{\prime}$.

(2) In particular, if the hard Lefschetz conjecture (Conjecture 2.4) holds for $\left(X^{\prime}, L^{\prime}\right)$ for all ample $\mathbb{R}$-divisors $L^{\prime}$ on $X^{\prime}$, then the Hodge standard conjecture (Conjecture [2.6) holds for $\left(X^{\prime}, L^{\prime}\right)$ for all ample $\mathbb{R}$-divisors $L^{\prime}$ on $X^{\prime}$.

Proof. By Corollary 2.12, the assertion easily follows from Proposition 3.4.

Remark 3.7. Our proof of Proposition 3.4 is based on a consideration of the asymptotic behavior of the cup product pairing as $\varepsilon \rightarrow 0$. Therefore, by our method, we can not prove whether we can take $\beta=\alpha$. In other words, we can not prove the Hodge standard conjecture (Conjecture 2.6) for all ample $\mathbb{R}$-divisors $L^{\prime}$ on $X^{\prime}$ of the form $L^{\prime}=f^{*} \mathscr{O}(L)-\varepsilon Y^{\prime}$. 


\section{SOME BLOWING-UPS OF PROJECTIVE SPACES}

In this section, we consider a projective smooth variety $B^{n}$ of dimension $n$ over $\overline{\mathbb{F}}_{q}$ which is obtained by successive blowing-ups of $\mathbb{P}_{\overline{\mathbb{F}}_{q}}^{n}$ along linear subvarieties. All varieties appearing in this section are defined over $\overline{\mathbb{F}}_{q}$ and satisfy Assumption 2.1 in $\$ 2.1$ Hence, for simplicity, we omit the subscript " $\overline{\mathbb{F}}_{q}$ " and denote $\mathbb{P}_{\overline{\mathbb{F}}_{q}}^{n}$ by $\mathbb{P}^{n}$, etc. This does not cause any confusion. We use the same notation as in 2.1 .

4.1. Construction of $B^{n}$. Let $n \geq 1$ be an integer. We have a natural map $\varphi: \mathbb{A}^{n+1} \backslash\{0\} \rightarrow \mathbb{P}^{n}$. A subvariety $V \subset \mathbb{P}^{n}$ is called a linear subvariety if $\varphi^{-1}(V) \cup$ $\{0\} \subset \mathbb{A}^{n+1}$ is a linear subspace. Let $\mathrm{Gr}_{d}\left(\mathbb{P}^{n}\right)$ be the Grassmann variety of linear subvarieties of dimension $d$ in $\mathbb{P}^{n}$. Let $\mathrm{Gr}_{*}\left(\mathbb{P}^{n}\right)$ be the disjoint union of $\mathrm{Gr}_{d}\left(\mathbb{P}^{n}\right)$ for all $d . \operatorname{Gr}_{d}\left(\mathbb{P}^{n}\right)\left(\mathbb{F}_{q}\right)$ is the set of linear subvarieties of dimension $d$ in $\mathbb{P}^{n}$ defined over $\mathbb{F}_{q}$.

We construct a birational map $f: B^{n} \rightarrow \mathbb{P}^{n}$ as follows. Firstly, we put $Y_{0}:=\mathbb{P}^{n}$. Then, let $Y_{1}$ be the blow-up of $Y_{0}$ along the disjoint union of all $\mathbb{F}_{q}$-rational points on $\mathbb{P}^{n}$. Let $Y_{2}$ be the blow-up of $Y_{1}$ along disjoint union of all strict transforms of lines in $\mathbb{P}^{n}$. Similarly, we construct projective smooth varieties $Y_{0}, \ldots, Y_{n-1}$ inductively as follows. Assume that $Y_{k}$ was already constructed. Let $Z_{k} \subset Y_{k}$ be the union of all strict transforms of linear subvarieties of dimension $k$ in $\mathbb{P}^{n}$ defined over $\mathbb{F}_{q}$. These are disjoint because all intersections of them were already blown-up. Let $g_{k}: Y_{k+1} \rightarrow Y_{k}$ be the blow-up of $Y_{k}$ along $Z_{k}$. Finally, we put $B^{n}:=Y_{n-1}$ and $f:=g_{0} \circ \cdots \circ g_{n-2}: B^{n} \rightarrow \mathbb{P}^{n}$. We have the following sequence of blowing-ups.

$$
B^{n}=Y_{n-1} \stackrel{g_{n-2}}{\longrightarrow} Y_{n-2} \stackrel{g_{n-3}}{\longrightarrow} \cdots \stackrel{g_{1}}{\longrightarrow} Y_{1} \stackrel{g_{0}}{\longrightarrow} Y_{0}=\mathbb{P}^{n}
$$

Note that there is a natural action of $\mathrm{PGL}_{n+1}\left(\mathbb{F}_{q}\right)$ on $\mathbb{P}^{n}$, and the above construction is equivariant with respect to $\mathrm{PGL}_{n+1}\left(\mathbb{F}_{q}\right)$-action. Hence we have a natural action of $\mathrm{PGL}_{n+1}\left(\mathbb{F}_{q}\right)$ on $B^{n}$.

4.2. Divisors on $B^{n}$. For $V \in \operatorname{Gr}_{k}\left(\mathbb{P}^{n}\right)\left(\mathbb{F}_{q}\right)$, we define a smooth irreducible divisor $D_{V}$ on $B^{n}$ as follows. If $k=n-1$, let $D_{V} \subset B^{n}$ be the strict transform of $V$. If $k<n-1$, the strict transform $\widetilde{V} \subset Y_{k}$ of $V$ is a connected component of $Z_{k}$. Therefore, $g_{k}^{-1}(\widetilde{V}) \subset Y_{k+1}$ is a $\mathbb{P}^{n-d-1}$-bundle over $\widetilde{V}$. Let $D_{V} \subset B^{n}$ be the strict transform of $g_{k}^{-1}(\widetilde{V})$.

By construction, (3.3.3) and induction, an $\mathbb{R}$-divisor $D$ on $B^{n}$ is written uniquely as

$$
D=f^{*} \mathscr{O}(H)+\sum_{\substack{V \in \operatorname{Gr}_{k}\left(\mathbb{P}^{n}\right)\left(\mathbb{F}_{q}\right), 0 \leq k \leq n-2}} a_{V} D_{V} \quad \text { in } \quad H^{2}\left(B^{n}\right)
$$


where $H$ is an $\mathbb{R}$-divisor on $\mathbb{P}^{n}$, and $a_{V} \in \mathbb{R}$. We also see that, all $\mathbb{R}$-divisors $D^{\prime}$ on $B^{n}$ is written as

$$
D^{\prime}=\sum_{V \in \mathrm{Gr}_{*}\left(\mathbb{P}^{n}\right)\left(\mathbb{F}_{q}\right)} a_{V}^{\prime} D_{V} \quad\left(a_{V}^{\prime} \in \mathbb{R}\right) \quad \text { in } \quad H^{2}\left(B^{n}\right) .
$$

Note that this second expression is not unique.

For $0 \leq k \leq n-1$, we define a divisor $D_{k}$ on $B^{n}$ by

$$
D_{k}:=\sum_{V \in \mathrm{Gr}_{k}\left(\mathbb{P}^{n}\right)\left(\mathbb{F}_{q}\right)} D_{V} .
$$

By construction, we see that the support of $D_{k}$ is a disjoint union of smooth divisors on $B^{n}$.

In this section, we use the following terminology for $\mathbb{R}$-divisors on $B^{n}$.

Definition 4.1. Let $D$ be an $\mathbb{R}$-divisor on $B^{n}$.

(1) $D$ is called positive if $D$ can be written as

$$
D=\sum_{V \in \mathrm{Gr}_{*}\left(\mathbb{P}^{n}\right)\left(\mathbb{F}_{q}\right)} a_{V} D_{V} \quad\left(a_{V} \in \mathbb{R}_{>0}\right) \quad \text { in } \quad H^{2}\left(B^{n}\right) .
$$

(2) $D$ is called $\mathrm{PGL}_{n+1}\left(\mathbb{F}_{q}\right)$-invariant if the class of $D$ in $H^{2}\left(B^{n}\right)$ is $\operatorname{PGL}_{n+1}\left(\mathbb{F}_{q}\right)$ invariant.

It is easy to see that $D$ is $\mathrm{PGL}_{n+1}\left(\mathbb{F}_{q}\right)$-invariant if and only if $D$ can be written as $D=f^{*} \mathscr{O}(H)+\sum_{k=0}^{n-1} b_{k} D_{k}$ in $H^{2}\left(B^{n}\right)$ for some $H, b_{k}$ (for a similar criterion of positive divisors, see Proposition 4.4).

4.3. Intersections of divisors $D_{V}$ on $B^{n}$. Here we study the combinatorial structure of intersections of divisors $D_{V}$ on $B^{n}$ (see also Mus, proof of Theorem $4.1, \mathrm{I})$.

Proposition 4.2. (1) For $V, W \in \mathrm{Gr}_{*}\left(\mathbb{P}^{n}\right)\left(\mathbb{F}_{q}\right), D_{V} \cap D_{W} \neq \emptyset$ if and only if $V \subset W$ or $W \subset V$.

(2) For $V \in \operatorname{Gr}_{d}\left(\mathbb{P}^{n}\right)\left(\mathbb{F}_{q}\right)$, we have a noncanonical isomorphism

$$
D_{V} \cong B^{d} \times B^{n-d-1}
$$

where $B^{d}$ (resp. $\left.B^{n-d-1}\right)$ is a variety constructed by successive blowingups of $\mathbb{P}^{d}$ (resp. $\left.\mathbb{P}^{n-d-1}\right)$ by the same way as $B^{n}$.

(3) Let $x \in V$ be a point which does not lie in any linear subvariety defined over $\mathbb{F}_{q}$ strictly contained in $V$. Let $\widetilde{V} \subset Y_{d}$ be the strict transform of $V$. Then $g_{d}: g_{d}^{-1}(\widetilde{V}) \rightarrow \widetilde{V}$ is a trivial $\mathbb{P}^{n-d-1}$-bundle. Moreover, $\widetilde{V}$ is isomorphic to $B^{d}$, and an isomorphism $D_{V} \cong B^{d} \times B^{n-d-1}$ as in 2. is induced from a choice of $x \in V$ and a trivialization $g_{d}^{-1}(\widetilde{V}) \cong \widetilde{V} \times \mathbb{P}\left(\check{N}_{V / \mathbb{P}^{n}, x}\right)$, where $\check{N}_{V / \mathbb{P}^{n}, x}$ is the fiber at $x$ of the conormal bundle $\check{N}_{V / \mathbb{P}^{n}}$. 
(4) For $W \in \operatorname{Gr}_{*}\left(\mathbb{P}^{n}\right)\left(\mathbb{F}_{q}\right)$ strictly contained in $V, D_{W}$ intersects transversally with $D_{V}$, and the intersection $D_{W} \cap D_{V}$ is of the form

$$
D_{W} \cap D_{V}=\widetilde{D}_{W} \times B^{n-d-1} \quad \text { on } \quad D_{V},
$$

where $\widetilde{D}_{W}$ is a divisor on $B^{d}$ corresponding to the inclusion $W \hookrightarrow V \cong \mathbb{P}^{d}$.

(5) For $W^{\prime} \in \mathrm{Gr}_{*}\left(\mathbb{P}^{n}\right)\left(\mathbb{F}_{q}\right)$ strictly containing $V$, $D_{W^{\prime}}$ intersects transversally with $D_{V}$, and the intersection $D_{W^{\prime}} \cap D_{V}$ is of the form

$$
D_{W^{\prime}} \cap D_{V}=B^{d} \times \widetilde{\widetilde{D}}_{W^{\prime}} \quad \text { on } \quad D_{V}
$$

where $\widetilde{\widetilde{D}}_{W^{\prime}}$ is a divisor on $B^{n-d-1}$ corresponding to the inclusion $\mathbb{P}\left(\check{N}_{V / W^{\prime}, x}\right) \hookrightarrow$ $\mathbb{P}\left(\check{N}_{V / \mathbb{P}^{n}, x}\right) \cong \mathbb{P}^{n-d-1}$.

Proof. Firstly, we shall prove the first assertion. If $V \subset W$ or $W \subset V$, it is clear that $D_{V} \cap D_{W} \neq \emptyset$. Assume that $V \cap W$ is strictly smaller than $V, W$. Then, after blowing-up along the strict transform of $V \cap W$ in the construction of $B^{n}$, the strict transforms of $V$ and $W$ become disjoint. Therefore, $D_{V}$ does not intersect with $D_{W}$.

Recall that, for a vector bundle $E$ of rank $k$ over a smooth variety $X, \mathbb{P}(E)$ is a trivial $\mathbb{P}^{k-1}$-bundle if and only if $E \otimes_{\mathscr{O}_{X}} \mathscr{L}$ is a trivial vector bundle for a line bundle $\mathscr{L}$ over $X$ ([Hart2, II, Exercise 7.10). We call such $E$ a twist of a trivial vector bundle over $X$. For $V \in \operatorname{Gr}_{d}\left(\mathbb{P}^{n}\right)\left(\mathbb{F}_{q}\right)$, the normal bundle $N_{V / \mathbb{P}^{n}} \cong \mathscr{O}_{V}(1)^{\oplus n-d}$ is a twist of a trivial vector bundle over $V$. Hence the normal bundle $N_{\widetilde{V} / Y_{d}}$ is also a twist of a trivial vector bundle over $Y_{d}$, where $\widetilde{V} \subset Y_{d}$ is the strict transform of $V$ ( $\left[\mathrm{Fu}\right.$, B.6.10). Therefore, $g_{d}^{-1}(\widetilde{V})$ is a trivial $\mathbb{P}^{n-d-1}$-bundle over $\widetilde{V}$.

Here $\widetilde{V}$ is isomorphic to $B^{d}$. This follows from the fact that, for a sequence of regular embeddings $Z \hookrightarrow Y \hookrightarrow X$, the strict transform of $Y$ in the blow-up of $X$ along $Z$ is isomorphic to the blow-up of $Y$ along $Z$ ([Fu , B.6.9).

We fix a point $x \in V$ which does not lie in any linear subvariety defined over $\mathbb{F}_{q}$ strictly contained in $V$, and a trivialization $g_{d}^{-1}(\widetilde{V}) \cong \widetilde{V} \times \mathbb{P}\left(\check{N}_{V / \mathbb{P}^{n}, x}\right)$, where $\check{N}_{V / \mathbb{P}^{n}, x}$ denotes the fiber at $x$ of the conormal bundle $\check{N}_{V / \mathbb{P}^{n}}$. For $W \in$ $\mathrm{Gr}_{*}\left(\mathbb{P}^{n}\right)\left(\mathbb{F}_{q}\right)$ strictly containing $V$, a natural inclusion $V \subset W \subset \mathbb{P}^{n}$ induces $N_{V / W} \subset N_{V / \mathbb{P} n}$ and hence $\mathbb{P}\left(\check{N}_{V / W}\right) \subset \mathbb{P}\left(\check{N}_{V / \mathbb{P}^{n}}\right)$. Thus, $\mathbb{P}\left(\check{N}_{V / W, x}\right)$ is a linear subvariety of $\mathbb{P}\left(\check{N}_{V / \mathbb{P}^{n}, x}\right)$. From this, we see that the blowing-up of $g_{d}^{-1}(\widetilde{V})$ along the strict transform of a linear subvariety strictly containing $V$ corresponds to the blowing-up along the strict transform of a linear subvariety of the second factor $\mathbb{P}\left(\check{N}_{V / \mathbb{P}^{n}, x}\right)$ of $g_{d}^{-1}(\widetilde{V}) \cong \widetilde{V} \times \mathbb{P}\left(\check{N}_{V / \mathbb{P}^{n}, x}\right)$. Therefore, we have an isomorphism $D_{V} \cong B^{d} \times B^{n-d-1}$. The remaining assertions follow from the construction of this isomorphism.

Corollary 4.3. Let $H$ be an $\mathbb{R}$-divisor on $\mathbb{P}^{n}, V \in \operatorname{Gr}_{d}\left(\mathbb{P}^{n}\right)\left(\mathbb{F}_{q}\right)$, and $f: B^{n} \rightarrow$ $\mathbb{P}^{n}$ a natural map as in $\$ 4.1$. Fix an isomorphism $D_{V} \cong B^{d} \times B^{n-d-1}$ as in 
Proposition 4.2. Let $\operatorname{pr}_{1}: D_{V} \rightarrow B^{d}$ be the projection to the first factor. Let $f^{\prime}: B^{d} \rightarrow \mathbb{P}^{d} \cong V$ be a map defined similarly as $f$. Then we have

$$
\left.\left(f^{*} \mathscr{O}(H)\right)\right|_{D_{V}}=\operatorname{pr}_{1}^{*}\left(f^{\prime *}\left(\left.\mathscr{O}(H)\right|_{V}\right)\right) \quad \text { in } \quad H^{2}\left(D_{V}\right)
$$

Proof. This follows from the construction of an isomorphism $D_{V} \cong B^{d} \times B^{n-d-1}$ in Proposition 4.2 .

\section{4. $\mathrm{PGL}_{n+1}\left(\mathbb{F}_{q}\right)$-invariant $\mathbb{R}$-divisors on $B^{n}$.}

Proposition 4.4. (1) For $V \in \mathrm{Gr}_{n-1}\left(\mathbb{P}^{n}\right)\left(\mathbb{F}_{q}\right)$, we have

$$
f^{*} \mathscr{O}(V)=\sum_{\substack{W \in \mathrm{Gr}_{*}\left(\mathbb{P}^{n}\right)\left(\mathbb{F}_{q}\right), W \subset V}} D_{W} \quad \text { in } \quad H^{2}\left(B^{n}\right) .
$$

(2) For an $\mathbb{R}$-divisor $H$ on $\mathbb{P}^{n}, f^{*} \mathscr{O}(H)$ is $\mathrm{PGL}_{n+1}\left(\mathbb{F}_{q}\right)$-invariant.

(3) Let $D$ be a $\mathrm{PGL}_{n+1}\left(\mathbb{F}_{q}\right)$-invariant $\mathbb{R}$-divisor on $B^{n}$ which is written as

$$
D=\alpha f^{*} \mathscr{O}_{\mathbb{P}^{n}}(1)+\sum_{d=0}^{n-1} a_{d} D_{d} \quad\left(\alpha, a_{d} \in \mathbb{R}\right) \quad \text { in } \quad H^{2}\left(B^{n}\right) .
$$

Then, $D$ is positive if and only if $\alpha>0$ and

$$
a_{d}+\alpha \frac{\left|\mathbb{P}^{n-d-1}\left(\mathbb{F}_{q}\right)\right|}{\left|\mathbb{P}^{n}\left(\mathbb{F}_{q}\right)\right|}>0 \quad \text { for all } \quad 0 \leq d \leq n-1 .
$$

(4) Let $D$ be a $\mathrm{PGL}_{n+1}\left(\mathbb{F}_{q}\right)$-invariant $\mathbb{R}$-divisor on $B^{n}$. Take $V \in \operatorname{Gr}_{d}\left(\mathbb{P}^{n}\right)\left(\mathbb{F}_{q}\right)$, and fix an isomorphism $D_{V} \cong B^{d} \times B^{n-d-1}$ as in Proposition 4.2. Let $\mathrm{pr}_{1}: D_{V} \rightarrow B^{d}, \mathrm{pr}_{2}: D_{V} \rightarrow B^{n-d-1}$ be projections. Then, there exist a $\mathrm{PGL}_{d+1}\left(\mathbb{F}_{q}\right)$-invariant $\mathbb{R}$-divisor $D^{\prime}$ on $B^{d}$ and a $\mathrm{PGL}_{n-d}\left(\mathbb{F}_{q}\right)$-invariant $\mathbb{R}$-divisor $D^{\prime \prime}$ on $B^{n-d-1}$ such that

$$
\left.\mathscr{O}(D)\right|_{D_{V}}=\operatorname{pr}_{1}^{*} \mathscr{O}\left(D^{\prime}\right)+\operatorname{pr}_{2}^{*} \mathscr{O}\left(D^{\prime \prime}\right) \quad \text { in } \quad H^{2}\left(D_{V}\right)
$$

Proof. The first assertion follows from the fact that the multiplicity of $V$ along $W \in \mathrm{Gr}_{*}\left(\mathbb{P}^{n}\right)\left(\mathbb{F}_{q}\right)$ with $W \subset V$ is equal to 1 .

The second assertion is obvious because $\mathrm{PGL}_{n+1}\left(\mathbb{F}_{q}\right)$ acts trivially on $H^{2}\left(\mathbb{P}^{n}\right)$ and $f^{*}$ is $\mathrm{PGL}_{n+1}\left(\mathbb{F}_{q}\right)$-equivariant.

We shall compute $f^{*} \mathscr{O}(H)$ explicitly. Since $H^{2}\left(\mathbb{P}^{n}\right)$ is generated by the class of $\mathscr{O}_{\mathbb{P}^{n}}(1)$, we write $H=\alpha \mathscr{O}_{\mathbb{P}^{n}}(1)$ in $H^{2}\left(\mathbb{P}^{n}\right)$ for some $\alpha \in \mathbb{R}$. Since $\left|\mathrm{Gr}_{n-1}\left(\mathbb{P}^{n}\right)\left(\mathbb{F}_{q}\right)\right|=$ $\left|\mathbb{P}^{n}\left(\mathbb{F}_{q}\right)\right|$, and, for each $W \in \operatorname{Gr}_{d}\left(\mathbb{P}^{n}\right)\left(\mathbb{F}_{q}\right)$, there exist $\left|\mathbb{P}^{n-d-1}\left(\mathbb{F}_{q}\right)\right|$ elements in 
$\operatorname{Gr}_{n-1}\left(\mathbb{P}^{n}\right)\left(\mathbb{F}_{q}\right)$ containing $W$, we have the following equality in $H^{2}\left(B^{n}\right)$ :

$$
\begin{aligned}
f^{*} \mathscr{O}(H) & =\frac{\alpha}{\left|\mathbb{P}^{n}\left(\mathbb{F}_{q}\right)\right|} \sum_{V \in \mathrm{Gr}_{n-1}\left(\mathbb{P}^{n}\right)\left(\mathbb{F}_{q}\right)}\left(\sum_{\substack{W \in \mathrm{Gr}_{*}\left(\mathbb{P}^{n}\right)\left(\mathbb{F}_{q}\right), W \subset V}} D_{W}\right) \\
& =\frac{\alpha}{\left|\mathbb{P}^{n}\left(\mathbb{F}_{q}\right)\right|} \sum_{d=0}^{n-1}\left|\mathbb{P}^{n-d-1}\left(\mathbb{F}_{q}\right)\right| D_{d} .
\end{aligned}
$$

Of course, from this expression, we can check that $f^{*} \mathscr{O}(H)$ is $\operatorname{PGL}_{n+1}\left(\mathbb{F}_{q}\right)$ invariant.

The third assertion easily follows from the above computation because

$$
D=\sum_{d=0}^{n-1}\left(a_{d}+\alpha \frac{\left|\mathbb{P}^{n-d-1}\left(\mathbb{F}_{q}\right)\right|}{\left|\mathbb{P}^{n}\left(\mathbb{F}_{q}\right)\right|}\right) D_{d} \quad \text { in } \quad H^{2}\left(B^{n}\right) .
$$

Finally, we shall prove the last assertion. To avoid self-intersection, we write $D$ in the following form

$$
D=\alpha f^{*} \mathscr{O}_{\mathbb{P}^{n}}(1)+\sum_{0 \leq k \leq n-1, k \neq d} a_{k} D_{k} \quad\left(\alpha, a_{k} \in \mathbb{R}\right) \quad \text { in } \quad H^{2}\left(B^{n}\right) .
$$

It is enough to treat the case $D=\alpha f^{*} \mathscr{O}_{\mathbb{P}^{n}}(1)$ and $D=D_{k}(k \neq d)$ separately. The case $D=\alpha f^{*} \mathscr{O}_{\mathbb{P} n}(1)$ follows from Corollary 4.3 and the second assertion. The case $D=D_{k}(k \neq d)$ follows from Proposition 4.2 .

4.5. Ample $\mathrm{PGL}_{n+1}\left(\mathbb{F}_{q}\right)$-invariant $\mathbb{R}$-divisors on $B^{n}$. Here we study ample $\mathrm{PGL}_{n+1}\left(\mathbb{F}_{q}\right)$-invariant $\mathbb{R}$-divisors on $B^{n}$. First of all, we note that there exists at least one ample $\mathrm{PGL}_{n+1}\left(\mathbb{F}_{q}\right)$-invariant $\mathbb{R}$-divisor on $B^{n}$. It follows from the construction of $B^{n}$ in 4.1 and Proposition 3.2

Proposition 4.5. Let $D$ be an ample $\mathrm{PGL}_{n+1}\left(\mathbb{F}_{q}\right)$-invariant $\mathbb{R}$-divisor on $B^{n}$. Then, $D$ is positive (see Definition [4.1).

Proof. We prove the assertion by induction on $n$. The case $n=1$ is obvious. Assume that Proposition 4.5 was already proved in dimension $<n$.

Let $D$ be written uniquely as

$$
D=\alpha f^{*} \mathscr{O}_{\mathbb{P}^{n}}(1)+\sum_{d=0}^{n-2} a_{d} D_{d} \quad\left(\alpha, a_{d} \in \mathbb{R}\right) \quad \text { in } \quad H^{2}\left(B^{n}\right) .
$$

By Proposition 4.4, $D$ is positive if and only if $\alpha>0$ and

$$
a_{d}+\alpha \frac{\left|\mathbb{P}^{n-d-1}\left(\mathbb{F}_{q}\right)\right|}{\left|\mathbb{P}^{n}\left(\mathbb{F}_{q}\right)\right|}>0 \quad \text { for all } \quad 0 \leq d \leq n-2 .
$$


Fix $V \in \operatorname{Gr}_{n-1}\left(\mathbb{P}^{n}\right)\left(\mathbb{F}_{q}\right)$. By Proposition 4.2, $D_{V}$ is isomorphic to $B^{n-1}$. We consider the restriction of $\mathscr{O}(D)$ to $D_{V}$. Let $f^{\prime}: D_{V} \cong B^{n-1} \rightarrow \mathbb{P}^{n-1}$ be a map defined similarly as $f$. Then, by Proposition 4.2 and Corollary 4.3, we have

$$
\left.\mathscr{O}(D)\right|_{D_{V}}=\alpha f^{\prime *} \mathscr{O}_{\mathbb{P}^{n-1}}(1)+\sum_{d=0}^{n-2} a_{d} D_{d}^{\prime} \quad \text { in } \quad H^{2}\left(B^{n}\right),
$$

where $D_{d}^{\prime}$ is a divisor on $D_{V} \cong B^{n-1}$ defined similarly as $D_{d}$ on $B^{n}$.

By induction, $\left.\mathscr{O}(D)\right|_{D_{V}}$ is positive. Therefore, by Proposition 4.4, we have $\alpha>0$ and

$$
a_{d}+\alpha \frac{\left|\mathbb{P}^{n-d-2}\left(\mathbb{F}_{q}\right)\right|}{\left|\mathbb{P}^{n-1}\left(\mathbb{F}_{q}\right)\right|}>0 \quad \text { for all } \quad 0 \leq d \leq n-2 .
$$

Therefore, it is enough to show the following inequality

$$
\frac{\left|\mathbb{P}^{n-d-1}\left(\mathbb{F}_{q}\right)\right|}{\left|\mathbb{P}^{n}\left(\mathbb{F}_{q}\right)\right|}>\frac{\left|\mathbb{P}^{n-d-2}\left(\mathbb{F}_{q}\right)\right|}{\left|\mathbb{P}^{n-1}\left(\mathbb{F}_{q}\right)\right|}
$$

Since $\left|\mathbb{P}^{k}\left(\mathbb{F}_{q}\right)\right|=\frac{q^{k+1}-1}{q-1}$, the above inequality is equivalent to

$$
\begin{aligned}
& \frac{q^{n-d}-1}{q^{n+1}-1}>\frac{q^{n-d-1}-1}{q^{n}-1} \\
& \quad \Longleftrightarrow q^{2 n-d}-q^{n}-q^{n-d}+1>q^{2 n-d}-q^{n+1}-q^{n-d-1}+1 \\
& \quad \Longleftrightarrow q^{n+1}+q^{n-d-1}>q^{n}+q^{n-d} \\
& \quad \Longleftrightarrow q^{n}(q-1)>q^{n-d-1}(q-1) .
\end{aligned}
$$

Hence we prove Proposition 4.5 .

\subsection{Hodge standard conjecture for $B^{n}$ with ample $\mathrm{PGL}_{n+1}\left(\mathbb{F}_{q}\right)$-invariant $\mathbb{R}$-divisors.}

Lemma 4.6. For $0 \leq k \leq n-1$ and $a \in H^{k}\left(B^{n}\right)$, if the restriction of a to $D_{V}$ is zero for all $V \in \mathrm{Gr}_{*}\left(\mathbb{P}^{n}\right)\left(\mathbb{F}_{q}\right)$, then $a=0$.

Proof. By applying Proposition 3.1 successively, we see that $a$ is of the form $a=f^{*} a^{\prime}$ for some $a^{\prime} \in H^{k}\left(\mathbb{P}^{n}\right)$. For $V \in \mathrm{Gr}_{n-1}\left(\mathbb{P}^{n}\right)\left(\mathbb{F}_{q}\right)$, the restriction of $a^{\prime}$ to $V$ is zero because the restriction of $a$ to $D_{V}$ is zero. Since restriction map $H^{k}\left(\mathbb{P}^{n}\right) \rightarrow H^{k}(V)$ is an isomorphism, we conclude that $a=0$.

The following proposition is the main result of this section.

Proposition 4.7. Let $D$ be an ample $\mathrm{PGL}_{n+1}\left(\mathbb{F}_{q}\right)$-invariant $\mathbb{R}$-divisor on $B^{n}$. Then the hard Lefschetz conjecture (Conjecture 2.4) and the Hodge standard conjecture (Conjecture [2.6) hold for $\left(B^{n}, D\right)$. 
Proof. We prove the assertion by induction on $n$. The case $n=1$ is obvious. Assume that Proposition 4.7 was already proved in dimension $<n$.

By the construction of $B^{n}$ in 4.1 and Proposition 3.4, we see that there exists at least one ample $\mathrm{PGL}_{n+1}\left(\mathbb{F}_{q}\right)$-invariant $\mathbb{R}$-divisor on $B^{n}$ for which Proposition 4.7 holds. If $D, D^{\prime}$ are ample $\mathrm{PGL}_{n+1}\left(\mathbb{F}_{q}\right)$-invariant $\mathbb{R}$-divisors on $B^{n}$, then $t D+$ $(1-t) D^{\prime}$ is also an ample $\mathrm{PGL}_{n+1}\left(\mathbb{F}_{q}\right)$-invariant $\mathbb{R}$-divisor on $B^{n}$ for $t \in \mathbb{R}$ with $0 \leq t \leq 1$. Therefore, by Corollary 2.12, it is enough to show that the hard Lefschetz conjecture (Conjecture 2.4) holds for $\left(B^{n}, D\right)$ for all ample $\mathrm{PGL}_{n+1}\left(\mathbb{F}_{q}\right)$ invariant $\mathbb{R}$-divisors $D$ on $B^{n}$.

Assume that there exists an ample $\mathrm{PGL}_{n+1}\left(\mathbb{F}_{q}\right)$-invariant $\mathbb{R}$-divisor $L$ on $B^{n}$ and a nonzero cohomology class $a \in H^{k}\left(B^{n}\right)$ for $0 \leq k \leq n-1$ such that

$$
L^{n-k} \cup a=0 .
$$

For $0 \leq d \leq n-1$, take $V \in \operatorname{Gr}_{d}\left(\mathbb{P}^{n}\right)\left(\mathbb{F}_{q}\right)$ and fix an isomorphism $D_{V} \cong$ $B^{d} \times B^{n-d-1}$ as in Proposition 4.2 Let $\mathrm{pr}_{1}: D_{V} \rightarrow B^{d}, \mathrm{pr}_{2}: D_{V} \rightarrow B^{n-d-1}$ be projections. Then, by Proposition 4.4, the restriction $\left.\mathscr{O}(L)\right|_{D_{V}}$ can be written as

$$
\left.\mathscr{O}(L)\right|_{D_{V}}=\operatorname{pr}_{1}^{*} \mathscr{O}\left(D^{\prime}\right)+\operatorname{pr}_{2}^{*} \mathscr{O}\left(D^{\prime \prime}\right) \quad \text { in } \quad H^{2}\left(D_{V}\right),
$$

where $D^{\prime}$ (resp. $D^{\prime \prime}$ ) is an ample $\operatorname{PGL}_{d+1}\left(\mathbb{F}_{q}\right)$-invariant (resp. $\mathrm{PGL}_{n-d}\left(\mathbb{F}_{q}\right)$ invariant) $\mathbb{R}$-divisor on $B^{d}$ (resp. $B^{n-d-1}$ ). Therefore, by induction hypothesis and Proposition 2.13, the hard Lefschetz conjecture (Conjecture 2.4) and the Hodge standard conjecture (Conjecture 2.6) hold for $\left(D_{V},\left.\mathscr{O}(L)\right|_{D_{V}}\right)$.

By restricting $L^{n-k} \cup a=0$ to $D_{V}$, we have $\left(\left.\mathscr{O}(L)\right|_{D_{V}}\right)^{n-k} \cup\left(\left.a\right|_{D_{V}}\right)=0$. Since $\operatorname{dim} D_{V}=n-1$, this implies $\left.a\right|_{D_{V}} \in H^{k}\left(D_{V}\right)$ is in the primitive part $P^{k}\left(D_{V}\right)$. Therefore, by the Hodge standard conjecture (Conjecture 2.6) for $\left(D_{V},\left.\mathscr{O}(L)\right|_{D_{V}}\right)$, we have

$$
(-1)^{k / 2} \cdot\left(\left.\mathscr{O}(L)\right|_{D_{V}}\right)^{n-k-1} \cup\left(\left.a\right|_{D_{V}}\right) \cup\left(\left.a\right|_{D_{V}}\right) \geq 0
$$

and the equality holds if and only if $\left.a\right|_{D_{V}}=0$.

By Proposition 4.5, $L$ can be written as

$$
L=\sum_{V \in \operatorname{Gr}_{*}\left(\mathbb{P}^{n}\right)\left(\mathbb{F}_{q}\right)} a_{V} D_{V} \quad\left(a_{V} \in \mathbb{R}_{>0}\right) \quad \text { in } H^{2}\left(B^{n}\right) .
$$

Therefore, we have

$$
\begin{aligned}
(-1)^{k / 2} \cdot L^{n-k} \cup a \cup a & \\
\quad & \sum_{V \in \mathrm{Gr}_{*}\left(\mathbb{P}^{n}\right)\left(\mathbb{F}_{q}\right)} a_{V} \cdot D_{V} \cup\left((-1)^{k / 2} \cdot L^{n-k-1} \cup a \cup a\right) \\
& =\sum_{V \in \mathrm{Gr}_{*}\left(\mathbb{P}^{n}\right)\left(\mathbb{F}_{q}\right)} a_{V}\left((-1)^{k / 2} \cdot\left(\left.\mathscr{O}(L)\right|_{D_{V}}\right)^{n-k-1} \cup\left(\left.a\right|_{D_{V}}\right) \cup\left(\left.a\right|_{D_{V}}\right)\right) \\
& =0
\end{aligned}
$$


because $L^{n-k} \cup a=0$. Since $a_{V}>0$ for all $V \in \mathrm{Gr}_{*}\left(\mathbb{P}^{n}\right)\left(\mathbb{F}_{q}\right)$, we have $\left.a\right|_{D_{V}}=0$ for all $V \in \operatorname{Gr}_{*}\left(\mathbb{P}^{n}\right)\left(\mathbb{F}_{q}\right)$. By Lemma 4.6, we have $a=0$. This is a contradiction. Hence the proof of Proposition 4.7] is complete.

Remark 4.8. Mustafin, Kurihara showed that $B^{d}$ is isomorphic to all irreducible components of the special fiber of the formal scheme model $\widehat{\Omega}_{\mathscr{O}_{K}}^{d}$ over $\operatorname{Spf} \mathscr{O}_{K}$ of the Drinfeld upper half space $\widehat{\Omega}_{K}^{d}$ of dimension $d$ (for details, see 86 , see also [Mus, $\mathrm{Ku}$ ). Moreover, they also showed that

$$
D=-(d+1) f^{*} \mathscr{O}_{\mathbb{P}^{d}}(1)+\sum_{k=0}^{d-1}(d-k) D_{k}
$$

is an ample divisor on $B^{d}$, and $D$ coincides with the restriction of the "relative dualizing sheaf" $\omega_{\widehat{\Omega}_{\mathscr{O}_{K}}^{d} / \mathscr{O}_{K}}$ to an irreducible component of the special fiber. These facts are crucial in our application to $p$-adic uniformization in $\$ 6$.

Remark 4.9. In our proof of Proposition 4.5 we heavily use the assumption that $D$ is $\mathrm{PGL}_{n+1}\left(\mathbb{F}_{q}\right)$-invariant. Therefore, our proof does not work for ample non-PGL $\mathrm{P}_{n+1}\left(\mathbb{F}_{q}\right)$-invariant $\mathbb{R}$-divisors on $B^{n}$. It is easy to see that if all ample $\mathbb{R}$-divisors on $B^{n}$ are positive, then Proposition 4.7 holds for them. However, the author does not even know whether it is natural to expect this. He has neither evidence nor counter-example for ample non-PGL $\mathrm{L}_{n+1}\left(\mathbb{F}_{q}\right)$-invariant $\mathbb{R}$-divisors.

\section{Review of the Weight SPECtral SEQUence of RAPoport-Zink}

In this section, we recall the definitions and basic properties of monodromy filtration, weight filtration, and the weight spectral sequence of Rapoport-Zink. Since we work over a local field in this section, we slightly change the notation. As in $₫ \mathbb{1}$ let $K$ be a complete discrete valuation field with finite residue field $\mathbb{F}_{q}$ of characteristic $p>0, l$ be a prime number different from $p$. Let $X$ be a proper smooth variety of dimension $n$ over $K$, and $V:=H_{\text {ét }}^{w}\left(X_{\bar{K}}, \mathbb{Q}_{l}\right)$.

5.1. Monodromy filtration. Let $I_{K}$ be the inertia group of $K$, which is a subgroup of $\operatorname{Gal}(\bar{K} / K)$ defined by the exact sequence:

$$
1 \longrightarrow I_{K} \longrightarrow \operatorname{Gal}(\bar{K} / K) \longrightarrow \operatorname{Gal}\left(\overline{\mathbb{F}}_{q} / \mathbb{F}_{q}\right) \longrightarrow 1 \text {. }
$$

$\operatorname{Gal}\left(\overline{\mathbb{F}}_{q} / \mathbb{F}_{q}\right)$ acts on $I_{K}$ by conjugation $\left(\tau: \sigma \mapsto \tau \sigma \tau^{-1}, \tau \in \operatorname{Gal}\left(\overline{\mathbb{F}}_{q} / \mathbb{F}_{q}\right), \sigma \in I_{K}\right)$. The pro-l-part of $I_{K}$ is isomorphic to $\mathbb{Z}_{l}(1)$ as a $\operatorname{Gal}\left(\overline{\mathbb{F}}_{q} / \mathbb{F}_{q}\right)$-module by

$$
t_{l}: I_{K} \ni \sigma \mapsto\left(\frac{\sigma\left(\pi^{1 / l^{m}}\right)}{\pi^{1 / l^{m}}}\right)_{m} \in \lim _{\longleftarrow} \mu_{l^{m}}=\mathbb{Z}_{l}(1),
$$

where $\pi$ is a uniformizer of $K$, and $\mu_{l^{m}}$ is the group of $l^{m}$-th roots of unity. It is known that $t_{l}$ is independent of the choice of $\pi$ and its $l^{m}$-th root $\pi^{1 / l^{m}}$ ([Se1]).

By Grothendieck's monodromy theorem ([ST], Appendix), there exist $r, s \geq 1$ such that $\left(\rho(\sigma)^{r}-1\right)^{s}=0$ for all $\sigma \in I_{K}$. Therefore, by replacing $K$ by its finite 
extension, $I_{K}$ acts on $V$ through $t_{l}: I_{K} \rightarrow \mathbb{Z}_{l}(1)$ and this action is unipotent. Then there is a unique nilpotent map of $\operatorname{Gal}(\bar{K} / K)$-representations called the monodromy operator $N: V(1) \rightarrow V$ such that

$$
\rho(\sigma)=\exp \left(t_{l}(\sigma) N\right) \text { for all } \sigma \in I_{K} .
$$

Here $N$ is a nilpotent map means that $N^{r}: V(r) \rightarrow V$ is zero for some $r \geq 1$.

Definition 5.1. ([De3, I, 1.7.2) There exists a unique filtration $M_{\bullet}$ on $V$ called the monodromy filtration characterized by the following properties.

(1) $M_{\bullet}$ is an increasing filtration $\cdots \subset M_{i-1} V \subset M_{i} V \subset M_{i+1} V \subset \cdots$ of $\operatorname{Gal}(\bar{K} / K)$-representations such that $M_{i} V=0$ for sufficiently small $i$ and $M_{i} V=V$ for sufficiently large $i$.

(2) $N\left(M_{i} V(1)\right) \subset M_{i-2} V$ for all $i$.

(3) By the second condition, we can define $N: \mathrm{Gr}_{i}^{M} V(1) \rightarrow \mathrm{Gr}_{i-2}^{M} V$, where $\mathrm{Gr}_{i}^{M} V:=M_{i} V / M_{i-1} V$. Then, for each $r \geq 0, N^{r}: \mathrm{Gr}_{r}^{M} V(r) \rightarrow \mathrm{Gr}_{-r}^{M} V$ is an isomorphism.

Remark 5.2. In the above definition, we replace $K$ by its finite extension. We can easily see that $M_{\bullet}$ is stable under the action of $\operatorname{Gal}(\bar{K} / K)$ for the original $K$. Therefore, we can define the monodromy filtration $M_{\bullet}$ as a filtration of $\operatorname{Gal}(\bar{K} / K)$-representations without replacing $K$ by its finite extension.

5.2. Weight filtration. Let $\operatorname{Fr}_{q} \in \operatorname{Gal}\left(\overline{\mathbb{F}}_{q} / \mathbb{F}_{q}\right)$ be the inverse of the $q$-th power map on $\overline{\mathbb{F}}_{q}$ called the geometric Frobenius element. A Gal $\left(\overline{\mathbb{F}}_{q} / \mathbb{F}_{q}\right)$-representation is said to have weight $k$ if all eigenvalues of the action of $\operatorname{Fr}_{q} \in \operatorname{Gal}\left(\overline{\mathbb{F}}_{q} / \mathbb{F}_{q}\right)$ are algebraic integers whose all complex conjugates have complex absolute value $q^{k / 2}$.

Definition 5.3. ([De1], [De3], I, 1.7.5) There exists a unique filtration $W$ •called the weight filtration on $V$ characterized by the following properties (for existence, see $\$ 5.31$.

(1) $W_{\bullet}$ is an increasing filtration $\cdots \subset W_{i-1} V \subset W_{i} V \subset W_{i+1} V \subset \cdots$ of $\operatorname{Gal}(\bar{K} / K)$-representations such that $W_{i} V=0$ for sufficiently small $i$ and $W_{i} V=V$ for sufficiently large $i$.

(2) For a lift $\widetilde{\operatorname{Fr}}_{q}$ of $\operatorname{Fr}_{q}$ in $\operatorname{Gal}(\bar{K} / K)$, all eigenvalues of the action of $\widetilde{\operatorname{Fr}}_{q}$ on each $\operatorname{Gr}_{i}^{W} V:=W_{i} V / W_{i-1} V$ are algebraic integers whose all complex conjugates have complex absolute value $q^{i / 2}$.

Remark 5.4. By the second condition, the monodromy operator $N: \operatorname{Gr}_{i}^{W} V(1) \rightarrow$ $\operatorname{Gr}_{i}^{W} V$ is zero for each $i$ (see 5 5.1). Hence, by replacing $K$ by its finite extension $K^{\prime}$ with residue field $\mathbb{F}_{q^{r}}, I_{K^{\prime}}$ acts on $\operatorname{Gr}_{i}^{W} V$ trivially, and the $\operatorname{Gal}\left(\overline{\mathbb{F}}_{q^{r}} / \mathbb{F}_{q^{r}}\right)$ representation $\operatorname{Gr}_{i}^{W} V$ has weight $i$ for each $i$.

\subsection{Weight spectral sequence of Rapoport-Zink.}


Definition 5.5. A regular scheme $\mathfrak{X}$ which is proper and flat over $\mathscr{O}_{K}$ is called a proper semistable model of $X$ over $\mathscr{O}_{K}$ if the generic fiber $\mathfrak{X}_{K}:=\mathfrak{X} \otimes_{\mathscr{O}_{K}} K$ is isomorphic to $X$ and the special fiber $\mathfrak{X}_{\mathbb{F}_{q}}:=\mathfrak{X} \otimes_{\mathscr{O}_{K}} \mathbb{F}_{q}$ is a divisor of $\mathfrak{X}$ with normal crossings. Moreover, if $\mathfrak{X}_{\mathbb{F}_{q}}$ is a divisor of $\mathfrak{X}$ with simple normal crossings, $\mathfrak{X}$ is called a proper strictly semistable model of $X$ over $\mathscr{O}_{K}$.

We recall the weight spectral sequence of Rapoport-Zink ([RZ1]). Assume that $X$ has a proper strictly semistable model $\mathfrak{X}$ over $\mathscr{O}_{K}$. Let $X_{1}, \ldots, X_{m}$ be the irreducible components of the special fiber of $\mathfrak{X}$, and

$$
X^{(k)}:=\coprod_{1 \leq i_{1}<\cdots<i_{k} \leq m} X_{i_{1}} \cap \cdots \cap X_{i_{k}} .
$$

Then $X^{(k)}$ is a disjoint union of proper smooth irreducible varieties of dimension $n-k+1$ over $F$. The weight spectral sequence of Rapoport-Zink is as follows:

$$
\begin{aligned}
E_{1}^{-r, w+r}=\bigoplus_{k \geq \max \{0,-r\}} H_{\text {ét }}^{w-r-2 k}\left(X_{\bar{F}_{q}}^{(2 k+r+1)}, \mathbb{Q}_{l}(-r-k)\right) & \\
& \Longrightarrow H_{\text {ét }}^{w}\left(X_{\bar{K}}, \mathbb{Q}_{l}\right) .
\end{aligned}
$$

This spectral sequence is $\operatorname{Gal}(\bar{K} / K)$-equivariant. The map $d_{1}^{i, j}: E_{1}^{i, j} \rightarrow E_{1}^{i+1, j}$ can be described in terms of restriction morphisms and Gysin morphisms explicitly (see [RZ1, 2.10 for details).

The action of the monodromy operator $N$ on $H_{\text {ét }}^{w}\left(X_{\bar{K}}, \mathbb{Q}_{l}\right)$ in $\$ 5.1$ is induced from a natural map $N: E_{1}^{i, j}(1) \rightarrow E_{1}^{i+2, j-2}$ satisfying

$$
N^{r}: E_{1}^{-r, w+r}(r) \stackrel{\cong}{\longrightarrow} E_{1}^{r, w-r}
$$

for all $r, w$. We can describe $N: E_{1}^{i, j}(1) \rightarrow E_{1}^{i+2, j-2}$ explicitly ([RZ1, 2.10). Hence we can define $N$ without replacing $K$ by its finite extension (see Remark 5.2).

The inertia group $I_{K}$ acts on each $E_{1}^{i, j}$ trivially and $\operatorname{Gal}\left(\overline{\mathbb{F}}_{q} / \mathbb{F}_{q}\right)$ acts on them. By the Weil conjecture ([De2], [De3] $), H_{\text {ét }}^{w-r-2 k}\left(X_{\overline{\mathbb{F}}_{q}}^{(2 k+r+1)}, \mathbb{Q}_{l}(-r-k)\right)$ has weight $(w-r-2 k)-2(-r-k)=w+r$ (see \$5.2). Hence $E_{1}^{i, j}$ has weight $j$. Therefore, the filtration on $H_{\text {ét }}^{w}\left(X_{\bar{K}}, \mathbb{Q}_{l}\right)$ induced by the weight spectral sequence is the weight filtration $W_{\bullet}$ in Definition 5.3 . This proves the existence of $W_{\bullet}$ in Definition 5.3 (for general $X$, we may use de Jong's alteration [d]). Moreover, since $d_{r}^{i, j}: E_{r}^{i, j} \rightarrow E_{r}^{i+r, j-r+1}$ is a map between $\operatorname{Gal}\left(\overline{\mathbb{F}}_{q} / \mathbb{F}_{q}\right)$-representations with different weights for $r \geq 2, d_{r}^{i, j}=0$ for $r \geq 2$. Therefore, the weight spectral sequence degenerates at $E_{2}$.

By combining above facts, we see that the weight-monodromy conjecture (Conjecture 1.1] is equivalent to the following conjecture on the weight spectral sequence of Rapoport-Zink. 
Conjecture 5.6 ([RZ1, [I1], [I12], [I13]). Let $X$ be a proper smooth variety over $K$ which has a proper strictly semistable model $\mathfrak{X}$ over $\mathscr{O}_{K}$. Let $E_{1}^{-r, w+r} \Rightarrow$ $H_{\text {ét }}^{w}\left(X_{\bar{K}}, \mathbb{Q}_{l}\right)$ be the weight spectral sequence of Rapoport-Zink. Then $N^{r}$ induces an isomorphism

$$
N^{r}: E_{2}^{-r, w+r}(r) \stackrel{\cong}{\longrightarrow} E_{2}^{r, w-r}
$$

on $E_{2}$-terms for all $r, w$.

\subsection{A positivity argument of Steenbrink, M. Saito.}

Proposition 5.7. Let $X$ be a proper smooth variety over $K$ which has a proper strictly semistable model $\mathfrak{X}$ over $\mathscr{O}_{K}$. Let $X_{1}, \ldots, X_{m}$ be the irreducible components of the special fiber of $\mathfrak{X}$. Assume that $\mathfrak{X}$ is projective over $\mathscr{O}_{K}$ with an ample line bundle $\mathscr{L}$. Assume further that, for $1 \leq i_{1}<\cdots<i_{k} \leq m$, each irreducible component $Y$ of $X_{i_{1}} \cap \cdots \cap X_{i_{k}}$ satisfies Assumption [2.1 in \$2.1, and the Hodge standard conjecture (Conjecture [2.6) holds for $\left(Y,\left.\mathscr{L}\right|_{Y}\right.$ ). Then, Conjecture 5.6 holds for $\mathfrak{X}$, and Conjecture 1.1 holds for $X$.

In the followings, we reduce the weight-monodromy conjecture (Conjecture 1.1, Conjecture [5.6) to an assertion that the restriction of a nondegenerate pairing to a subspace is nondegenerate (see Lemma 5.13 below). This method has a long story starting in SGA7, where Grothendieck considered the case of curves and abelian varieties ([SGA7-I], see also [11]).

The outline of the proof here is essentially the same as in [SaM1], 4.2.5, where M. Saito used polarized Hodge structures to prove a Hodge analogue of Conjecture 5.6] (see also SaM2]). The only difference is that we use $\mathbb{Q}$-structures on $l$-adic cohomology and the Hodge standard conjecture instead of the polarized Hodge structures (A similar argument can be found in It2]). Since the presentation of M. Saito in SaM1 seems too sophisticated, it is not very clear to non-specialists that the argument in SaM1] can also be applied to the situation in Proposition 5.7 even in positive or mixed characteristic. So we reproduce the argument here with slight modification suitable for Proposition 5.7 for the reader's convenience. Therefore, those who are familiar with the argument in SaM1 may skip to the next section.

First of all, we recall the structure of the $E_{1}$-terms of the weight spectral sequence of Rapoport-Zink. Let

$$
d_{1}^{-r, w+r}=\sum_{k \geq \max \{0,-r\}}(\rho+\tau): E_{1}^{-r, w+r} \longrightarrow E_{1}^{-r+1, w+r}
$$

denote the differential on $E_{1}$-terms, where

$$
\rho: H_{\text {ét }}^{s}\left(X_{\overline{\mathbb{F}}_{q}}^{(t)}, \mathbb{Q}_{l}\right) \longrightarrow H_{\text {ét }}^{s}\left(X_{\overline{\mathbb{F}}_{q}}^{(t+1)}, \mathbb{Q}_{l}\right)
$$


is a linear combination of restriction morphisms for some $s, t\left(\rho\right.$ is $(-1)^{r+k} \theta$ in RZ1, 2.10), and

$$
\tau: H_{\text {ét }}^{s}\left(X_{\overline{\mathbb{F}}_{q}}^{(t)}, \mathbb{Q}_{l}\right) \longrightarrow H_{\text {ét }}^{s+2}\left(X_{\overline{\mathbb{F}}_{q}}^{(t-1)}, \mathbb{Q}_{l}(1)\right)
$$

is a linear combination of Gysin morphisms for some $s, t\left(\tau\right.$ is $(-1)^{k} d_{1}^{\prime}$ in [RZ1, 2.10). From the construction, $\rho$ increases the index $k$ by 1 , and $\tau$ preserves the index $k$. Moreover, $\rho, \tau$ satisfy $\rho \circ \rho=0, \tau \circ \tau=0, \tau \circ \rho+\rho \circ \tau=0$. Hence $\rho, \tau$ also satisfy

$$
\rho \circ \tau \circ \rho=-\rho \circ \rho \circ \tau=0, \quad \tau \circ \rho \circ \tau=-\tau \circ \tau \circ \rho=0 .
$$

Let $k$ be an integer with $1 \leq k \leq n$. To distinguish $\rho, \tau$ for different degree, we use the following notation.

$$
\begin{aligned}
& \rho_{i}^{(k)}: H_{\text {ét }}^{i}\left(X_{\overline{\mathbb{F}}_{q}}^{(k)}, \mathbb{Q}_{l}\right) \longrightarrow H_{\text {ét }}^{i}\left(X_{\overline{\mathbb{F}}_{q}}^{(k+1)}, \mathbb{Q}_{l}\right), \\
& \tau_{i}^{(k+1)}: H_{\text {êt }}^{i}\left(X_{\overline{\mathbb{F}}_{q}}^{(k+1)}, \mathbb{Q}_{l}\right) \longrightarrow H_{\text {ét }}^{i+2}\left(X_{\overline{\mathbb{F}}_{q}}^{(k)}, \mathbb{Q}_{l}(1)\right) .
\end{aligned}
$$

In the followings, to simply the notation, we fix an isomorphism $\mathbb{Q}_{l} \cong \mathbb{Q}_{l}(1)$ to ignore the Tate twists, and simply write $H_{\text {ét }}^{i}\left(X^{(k)}\right):=H_{\text {ét }}^{i}\left(X_{\overline{\mathbb{F}}_{q}}^{(k)}, \mathbb{Q}_{l}\right)$, where we add a subscript "ét" not to confuse with the notation $H^{i}(X)$ in $\$ 2.1$.

Let $L: H_{\text {ét }}^{i}\left(X^{(k)}\right) \rightarrow H_{\text {ét }}^{i+2}\left(X^{(k)}\right)$ be the Lefschetz operator defined by $\mathscr{L}$. Since $L$ commutes with $\rho, \tau$, we use the same letter $L$ for different $i, k$, which does not cause any confusion. Let

$$
H_{\text {ét }}^{i}\left(X^{(k)}\right) \times H_{\text {ét }}^{2(n-k+1)-i}\left(X^{(k)}\right) \rightarrow \mathbb{Q}_{l}, \quad H_{\text {ét }}^{i}\left(X^{(k+1)}\right) \times H_{\text {ét }}^{2(n-k)-i}\left(X^{(k+1)}\right) \rightarrow \mathbb{Q}_{l}
$$

be the sum of the cup product pairings. For simplicity, we denote them by $\cup$. Then, for $a \in H_{\text {ét }}^{i}\left(X^{(k)}\right), \quad b \in H_{\text {ét }}^{2(n-k)-i}\left(X^{(k+1)}\right)$, we have

$$
a \cup \tau_{2(n-k)-i}^{(k+1)}(b)= \pm \rho_{i}^{(k)}(a) \cup b .
$$

Namely, $\rho_{i}^{(k)}$ and $\tau_{2(n-k)-i}^{(k+1)}$ are dual to each other with respect to $\cup$ up to sign. Here we do not specify the sign \pm because it does not matter in the following proof.

Lemma 5.8. In the following sequence of $\rho, \tau$

$$
H_{\text {ét }}^{i-2}\left(X^{(k+1)}\right) \stackrel{\tau_{i-2}^{(k+1)}}{\longrightarrow} H_{\text {ét }}^{i}\left(X^{(k)}\right) \stackrel{\rho_{i}^{(k)}}{\longrightarrow} H_{\text {ét }}^{i}\left(X^{(k+1)}\right) \stackrel{\tau_{i}^{(k+1)}}{\longrightarrow} H_{\text {ét }}^{i+2}\left(X^{(k)}\right),
$$

we have $\left(\operatorname{Ker} \tau_{i}^{(k+1)}\right) \cap\left(\operatorname{Im} \rho_{i}^{(k)}\right)=\operatorname{Im}\left(\rho_{i}^{(k)} \circ \tau_{i-2}^{(k+1)}\right)$. Moreover, in the following sequence of $\rho, \tau$

$$
H_{\text {ét }}^{i}\left(X^{(k)}\right) \stackrel{\rho_{i}^{(k)}}{\longrightarrow} H_{\text {ét }}^{i}\left(X^{(k+1)}\right) \stackrel{\tau_{i}^{(k+1)}}{\longrightarrow} H_{\text {ét }}^{i+2}\left(X^{(k)}\right) \stackrel{\rho_{i+2}^{(k)}}{\longrightarrow} H_{\text {ét }}^{i+2}\left(X^{(k+1)}\right),
$$

we have $\left(\operatorname{Ker} \rho_{i+2}^{(k)}\right) \cap\left(\operatorname{Im} \tau_{i}^{(k+1)}\right)=\operatorname{Im}\left(\tau_{i}^{(k+1)} \circ \rho_{i}^{(k)}\right)$.

By assuming the above lemma, it is easy to prove Proposition 5.7 . 
Proof of Proposition 5.7 assuming Lemma 5.8. For $r \geq 1$, we write $E_{1}^{-r, w+r}, E_{1}^{r, w-r}$ explicitly as follows (see $\$ 5.3$ )

$$
E_{1}^{-r, w+r}=\bigoplus_{k \geq 0} H_{\text {ét }}^{w-r-2 k}\left(X^{(2 k+r+1)}\right), \quad E_{1}^{r, w-r}=\bigoplus_{k \geq r} H_{\text {ét }}^{w+r-2 k}\left(X^{(2 k-r+1)}\right) .
$$

Put $k^{\prime}=k-r$ and rewrite $E_{1}^{r, w-r}$ as

$$
E_{1}^{r, w-r}=\bigoplus_{k^{\prime} \geq 0} H_{\text {ét }}^{w-r-2 k^{\prime}}\left(X^{\left(2 k^{\prime}+r+1\right)}\right) .
$$

From this expression, there is a natural isomorphism between $E_{1}^{-r, w+r}$ and $E_{1}^{r, w-r}$, which is nothing but an isomorphism induced by $N^{r}$ in $\$ 5.3$. Now, we look at the following part of the weight spectral sequence of Rapoport-Zink:

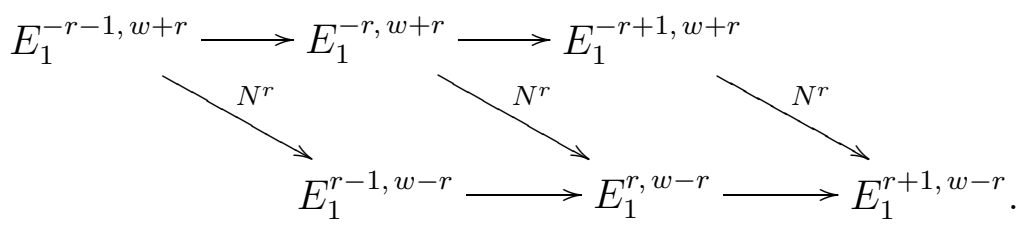

Note that $N^{r}$ is a morphism of complexes from the upper line to the lower one. It is easy to see that the leftmost $N^{r}$ is surjective, the middle $N^{r}$ is an isomorphism, and the rightmost $N^{r}$ is injective. To simplify the notation, we define

$$
\begin{aligned}
A & :=\bigoplus_{k \geq 0} H_{\text {ét }}^{w-r-2 k-2}\left(X^{(2 k+r+2)}\right), \\
B & :=\bigoplus_{k \geq 1} H_{\text {ét }}^{w-r-2 k}\left(X^{(2 k+r+1)}\right), \quad B^{\prime}:=H_{\text {ét }}^{w-r}\left(X^{(r+1)}\right), \\
C & :=\bigoplus_{k \geq 1} H_{\text {ét }}^{w-r-2 k+2}\left(X^{(2 k+r)}\right), \quad D:=H_{\text {ét }}^{w-r+2}\left(X^{(r)}\right), \quad E:=H_{\text {ét }}^{w-r}\left(X^{(r)}\right) .
\end{aligned}
$$

Then, (5.2) becomes

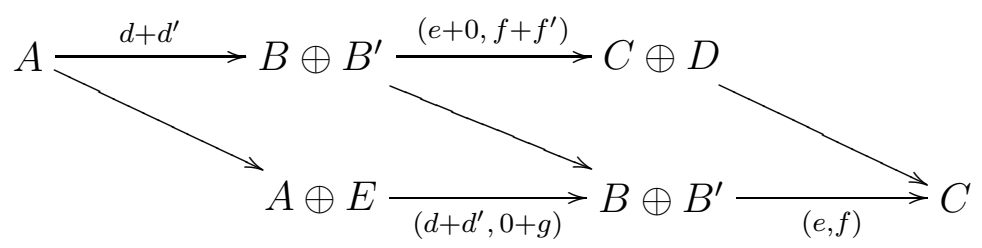

for some $d: A \rightarrow B, d^{\prime}: A \rightarrow B^{\prime}, e: B \rightarrow C, f: B^{\prime} \rightarrow C, f^{\prime}: B^{\prime} \rightarrow D, g: E \rightarrow$ $B^{\prime}$, where $B \oplus B^{\prime} \rightarrow B \oplus B^{\prime}$ is the identity map, and $A \rightarrow A \oplus E$ (resp. $C \oplus D \rightarrow C$ ) is a natural map $a \mapsto(a, 0)$ (resp. $(c, d) \mapsto c)$.

It is enough to show that the identity map $B \oplus B^{\prime} \rightarrow B \oplus B^{\prime}$ induces an isomorphism between the cohomologies of the first and second rows of (5.3).

We shall prove the injectivity. Take an element $\left(b, b^{\prime}\right) \in B \oplus B^{\prime}$ such that $e(b)+f\left(b^{\prime}\right)=0, f^{\prime}\left(b^{\prime}\right)=0$. Assume that $\left(b+b^{\prime}\right) \in \operatorname{Im}\left(d+d^{\prime}, 0+g\right)$. Namely, 
there exists $(x, y) \in A \oplus E$ such that $d(x)=b, d^{\prime}(x)+g(y)=b^{\prime}$. Then we have

$$
\begin{aligned}
e(b)+f\left(b^{\prime}\right) & =e \circ d(x)+f \circ d^{\prime}(x)+f \circ g(y)=0 \\
f^{\prime}\left(b^{\prime}\right) & =f^{\prime} \circ d^{\prime}(x)+f^{\prime} \circ g(y)=0 .
\end{aligned}
$$

From the definition, we see that $d^{\prime}=\tau_{w-r-2}^{(r+2)}, f^{\prime}=\tau_{w-r}^{(r+1)}, g=\rho_{w-r}^{(r)}$. Hence $f^{\prime} \circ d^{\prime}=\tau_{w-r}^{(r+1)} \circ \tau_{w-r-2}^{(r+2)}=0$. Therefore, we have

$$
f^{\prime} \circ g(y)=\tau_{w-r}^{(r+1)} \circ \rho_{w-r}^{(r)}(y)=0 .
$$

By the first assertion of Lemma [5.8, there exists $z \in H_{\text {ét }}^{w-r-2}\left(X^{(r+1)}\right)$ such that

$$
g(y)=\rho_{w-r}^{(r)}(y)=\rho_{w-r}^{(r)} \circ \tau_{w-r-2}^{(r+1)}(z) .
$$

Since $\rho_{w-r}^{(r)} \circ \tau_{w-r-2}^{(r+1)}(z)=-\tau_{w-r-2}^{(r+2)} \circ \rho_{w-r-2}^{(r+1)}(z)$, we have

$$
b^{\prime}=d^{\prime}(x)+g(y)=\tau_{w-r-2}^{(r+2)}(x)-\tau_{w-r-2}^{(r+2)} \circ \rho_{w-r-2}^{(r+1)}(z)=\tau_{w-r-2}^{(r+2)}\left(x-\rho_{w-r-2}^{(r+1)}(z)\right) .
$$

On the other hand, the restriction of $d: A \rightarrow B$ to the $k=0$ part $H_{\text {ét }}^{w-r-2}\left(X^{(r+2)}\right)$ is $\rho_{w-r-2}^{(r+2)}$. Then, we have

$$
d\left(x-\rho_{w-r-2}^{(r+1)}(z)\right)=d(x)-\rho_{w-r-2}^{(r+2)} \circ \rho_{w-r-2}^{(r+1)}(z)=d(x)=b,
$$

since $\rho_{w-r-2}^{(r+2)} \circ \rho_{w-r-2}^{(r+1)}=0$. Hence $\left(b, b^{\prime}\right)$ is the image of $x-\rho_{w-r-2}^{(r+1)}(z)$ by $d+d^{\prime}$, i.e. $\left(b, b^{\prime}\right) \in \operatorname{Im}\left(d+d^{\prime}\right)$. This proves the injectivity.

Finally, we shall prove the surjectivity. Take an element $\left(b, b^{\prime}\right) \in B \oplus B^{\prime}$ with $e(b)+f\left(b^{\prime}\right)=0$. From the construction, we see that $f=\rho_{w-r}^{(r+1)}$ and the restriction of $e: B \rightarrow C$ to the $k=1$ part $H_{\text {ét }}^{w-r-2}\left(X^{(r+3)}\right)$ is $\tau_{w-r-2}^{(r+3)}$. Therefore, the equality $e(b)+f\left(b^{\prime}\right)=0$ implies $\tau_{w-r-2}^{(r+3)}(\tilde{b})+\rho_{w-r}^{(r+1)}\left(b^{\prime}\right)=0$ in the $k=1$ part $H_{\text {ét }}^{w-r}\left(X^{(r+2)}\right)$ of $C$, where $\tilde{b}$ is the $k=1$ part of $b$. Hence we have

$$
\rho_{w-r+2}^{(r)} \circ \tau_{w-r}^{(r+1)}\left(b^{\prime}\right)=-\tau_{w-r}^{(r+2)} \circ \rho_{w-r}^{(r+1)}\left(b^{\prime}\right)=\tau_{w-r}^{(r+2)} \circ \tau_{w-r-2}^{(r+3)}(\tilde{b})=0,
$$

since $\rho_{w-r+2}^{(r)} \circ \tau_{w-r}^{(r+1)}=-\tau_{w-r}^{(r+2)} \circ \rho_{w-r}^{(r+1)}$ and $\tau_{w-r}^{(r+2)} \circ \tau_{w-r-2}^{(r+3)}=0$. By the second assertion of Lemma [5.8, there exists $a \in H_{\text {ét }}^{w-r}\left(X^{(r)}\right)=E$ such that

$$
\tau_{w-r}^{(r+1)}\left(b^{\prime}\right)=\tau_{w-r}^{(r+1)} \circ \rho_{w-r}^{(r)}(a) .
$$

Consider the element $\left(b, b^{\prime}-g(a)\right) \in B \oplus B^{\prime}$ which is in the same class as $\left(b, b^{\prime}\right)$ in the cohomology of the second row. Then, we have

$$
f^{\prime}\left(b^{\prime}-g(a)\right)=\tau_{w-r}^{(r+1)}\left(b^{\prime}\right)-\tau_{w-r}^{(r+1)} \circ \rho_{w-r}^{(r)}(a)=0,
$$

hence $\left(b, b^{\prime}-g(a)\right) \in \operatorname{Ker}(e+0, f+f)$. This proves the surjectivity.

Therefore, it is enough to prove Lemma 5.8. Firstly, recall the primitive decomposition of $H_{\text {ét }}^{i}\left(X^{(k)}\right)$ :

$$
H_{\text {ét }}^{i}\left(X^{(k)}\right)=\bigoplus_{j \geq 0} L^{j} P_{\text {ét }}^{i-2 j}\left(X^{(k)}\right),
$$


where $P_{\text {ét }}^{i-2 j}\left(X^{(k)}\right)$ is the primitive part of $H_{\text {ét }}^{i-2 j}\left(X^{(k)}\right)$ as in $\$ 2.2$. Recall that $\operatorname{dim} X^{(k)}=n-k+1$. The idea of our proof of Lemma 5.8 is to analyze the primitive decomposition carefully. Since $\rho, \tau$ do not preserve the primitive decomposition, for $0 \leq i \leq \operatorname{dim} X^{(k+1)}=n-k$, we put

$$
P^{i} \operatorname{Im}^{0} \rho_{i}^{(k)}:=\left(\operatorname{Im} \rho_{i}^{(k)}\right) \cap P_{\text {ét }}^{i}\left(X^{(k+1)}\right),
$$

and $P^{i} \operatorname{Im}^{0} \rho_{i}^{(k)}=0$ otherwise. Then, we define

$$
\operatorname{Im}^{0} \rho_{i}^{(k)}:=\bigoplus_{j \geq 0} L^{j} P^{i-2 j} \operatorname{Im}^{0} \rho_{i-2 j}, \quad \operatorname{Im}^{1} \rho_{i}^{(k)}:=\operatorname{Im} \rho_{i}^{(k)} / \operatorname{Im}^{0} \rho_{i}^{(k)} .
$$

Roughly speaking, $\operatorname{Im}^{0} \rho_{i}^{(k)}$ is a part which has a decomposition according to the primitive decomposition of $H_{\text {ét }}^{i}\left(X^{(k+1)}\right)$, and $\operatorname{Im}^{1} \rho_{i}^{(k)}$ is a part which does not have any decomposition compatible with the primitive decomposition of $H_{\text {ét }}^{i}\left(X^{(k+1)}\right)$. Similarly for $\operatorname{Im} \tau_{i}^{(k+1)}$, for $0 \leq i \leq \operatorname{dim} X^{(k)}-2=n-k-1$, we put

$$
P^{i+2} \operatorname{Im}^{0} \tau_{i}^{(k+1)}:=\operatorname{Im} \tau_{i}^{(k+1)} \cap P_{\text {ét }}^{i+2}\left(X^{(k)}\right)
$$

and $P^{i+2} \operatorname{Im}^{0} \tau_{i}^{(k+1)}=0$ otherwise. Then, we define

$$
\operatorname{Im}^{0} \tau_{i}^{(k+1)}:=\bigoplus_{j \geq 0} L^{j} P^{i+2-2 j} \operatorname{Im}^{0} \tau_{i-2 j}^{(k+1)}, \quad \operatorname{Im}^{1} \tau_{i}^{(k+1)}:=\operatorname{Im} \tau_{i}^{(k+1)} / \operatorname{Im}^{0} \tau_{i}^{(k+1)} .
$$

Lemma 5.9 (Hard Lefschetz theorem for $\mathrm{Im}^{0}$ ). We have the primitive decomposition for $\operatorname{Im}^{0}$ in the following sense:

$$
\begin{aligned}
& \operatorname{Im}^{0} \rho_{i}^{(k)}=\bigoplus_{j \geq 0}\left(L^{j} P_{\text {ét }}^{i-2 j}\left(X^{(k+1)}\right) \cap \operatorname{Im}^{0} \rho_{i}^{(k)}\right), \\
& \operatorname{Im}^{0} \tau_{i}^{(k+1)}=\bigoplus_{j \geq 0}\left(L^{j} P_{\text {ét }}^{i-2 j}\left(X^{(k)}\right) \cap \operatorname{Im}^{0} \tau_{i}^{(k+1)}\right) .
\end{aligned}
$$

Hence we have an analogue of the hard Lefschetz theorem for $\operatorname{Im}^{0}$ in the following form:

$$
\begin{aligned}
& L^{\operatorname{dim} X^{(k+1)}-i}=L^{(n-k)-i}: \operatorname{Im}^{0} \rho_{i}^{(k)} \quad \cong \quad \operatorname{Im}^{0} \rho_{2(n-k)-i}^{(k)}, \\
& L^{\operatorname{dim} X^{(k)}-(i+2)}=L^{(n-k+1)-(i+2)}: \operatorname{Im}^{0} \tau_{i}^{(k+1)} \stackrel{\cong}{\longrightarrow} \operatorname{Im}^{0} \tau_{2(n-k+1)-i-4}^{(k+1)} \text {. }
\end{aligned}
$$

Proof of Lemma 5.9. These immediately follows from the definition of $\operatorname{Im}^{0} \rho_{i}^{(k)}$, $\operatorname{Im}^{0} \tau_{i}^{(k+1)}$.

Lemma 5.10 (Hard Lefschetz theorem for $\mathrm{Im}^{1}$ ). We also have an analogue of the hard Lefschetz theorem for $\mathrm{Im}^{1}$ in the following sense:

$$
\begin{gathered}
L^{\operatorname{dim} X^{(k+1)}+1-i}=L^{(n-k)+1-i}: \operatorname{Im}^{1} \rho_{i}^{(k)} \stackrel{\cong}{\longrightarrow} \operatorname{Im}^{1} \rho_{2((n-k)+1)-i}^{(k)}, \\
L^{\operatorname{dim} X^{(k)}+1-(i+2)}=L^{(n-k+1)+1-(i+2)}: \operatorname{Im}^{1} \tau_{i}^{(k+1)} \stackrel{\cong}{\longrightarrow} \operatorname{Im}^{1} \tau_{2((n-k+1)+1)-(i+4)}^{(k+1)} .
\end{gathered}
$$


Proof of Lemma 5.10. The surjectivity follows from the hard Lefschetz theorem. The injectivity follows from the surjectivity of the maps

$$
\begin{gathered}
L^{(n-k)+1-i}: \operatorname{Im}^{0} \rho_{i}^{(k)} \\
L^{(n-k+1)+1-(i+2)}: \operatorname{Im}^{0} \tau_{i}^{(k+1)} \longrightarrow \operatorname{Im}^{0} \rho_{2((n-k)+1)-i}^{(k)} \\
\operatorname{Im}^{0} \tau_{2((n-k+1)+1)-(i+4)}^{(k+1)},
\end{gathered}
$$

which follows from Lemma 5.9 .

Remark 5.11. The primitive decomposition of $\operatorname{Im}^{0} \rho_{i}^{(k)}\left(\operatorname{resp} . \operatorname{Im}^{0} \tau_{i}^{(k+1)}\right)$ is the same as that of $H_{\text {ét }}^{i}\left(X^{(k+1)}\right)$ (resp. $H_{\text {ét }}^{i+2}\left(X^{(k)}\right)$ ). On the other hand, the primitive decomposition of $\operatorname{Im}^{1} \rho_{i}^{(k)}$ (resp. $\operatorname{Im}^{1} \tau_{i}^{(k+1)}$ ) is the same as that of $H_{\text {ét }}^{i}\left(X^{(k)}\right)$ (resp. $\left.H_{\text {ét }}^{i}\left(X^{(k+1)}\right)\right)$. Hence the center of symmetry of $\operatorname{Im}^{1}$ differs by 1 from that of $\operatorname{Im}^{0}$.

Lemma 5.12. For all $i$, we have the following equality of dimensions:

$$
\operatorname{dim}_{\mathbb{Q}_{l}} \operatorname{Im}^{0} \rho_{i}^{(k)}=\operatorname{dim}_{\mathbb{Q}_{l}} \operatorname{Im}^{1} \tau_{i}^{(k+1)}, \quad \operatorname{dim}_{\mathbb{Q}_{l}} \operatorname{Im}^{1} \rho_{i+2}^{(k)}=\operatorname{dim}_{\mathbb{Q}_{l}} \operatorname{Im}^{0} \tau_{i}^{(k+1)} .
$$

Proof of Lemma 5.12. Since $\rho_{i}^{(k)}$ and $\tau_{2(n-k)-i}^{(k+1)}$ are dual to each other up to sign (see (15.1)), we have $\operatorname{dim}_{\mathbb{Q}_{l}} \operatorname{Im} \rho_{i}^{(k)}=\operatorname{dim}_{\mathbb{Q}_{l}} \operatorname{Im} \tau_{2(n-k)-i}^{(k+1)}$. Hence we have

$$
\operatorname{dim}_{\mathbb{Q}_{l}} \operatorname{Im}^{0} \rho_{i}^{(k)}+\operatorname{dim}_{\mathbb{Q}_{l}} \operatorname{Im}^{1} \rho_{i}^{(k)}=\operatorname{dim}_{\mathbb{Q}_{l}} \operatorname{Im}^{0} \tau_{2(n-k)-i}^{(k+1)}+\operatorname{dim}_{\mathbb{Q}_{l}} \operatorname{Im}^{1} \tau_{2(n-k)-i}^{(k+1)}
$$

Since $\operatorname{dim}_{\mathbb{Q}_{l}} \operatorname{Im}^{0} \tau_{2(n-k)-i}^{(k+1)}=\operatorname{dim}_{\mathbb{Q}_{l}} \operatorname{Im}^{0} \tau_{i-2}^{(k+1)}$ by Lemma 5.9, and $\operatorname{dim}_{\mathbb{Q}_{l}} \operatorname{Im}^{1} \tau_{2(n-k)-i}^{(k+1)}=$ $\operatorname{dim}_{\mathbb{Q}_{l}} \operatorname{Im}^{1} \tau_{i}^{(k+1)}$ by Lemma 5.10, we have

$$
\operatorname{dim}_{\mathbb{Q}_{l}} \operatorname{Im}^{0} \rho_{i}^{(k)}+\operatorname{dim}_{\mathbb{Q}_{l}} \operatorname{Im}^{1} \rho_{i}^{(k)}=\operatorname{dim}_{\mathbb{Q}_{l}} \operatorname{Im}^{0} \tau_{i-2}^{(k+1)}+\operatorname{dim}_{\mathbb{Q}_{l}} \operatorname{Im}^{1} \tau_{i}^{(k+1)} .
$$

Equivalently, we have

$$
\operatorname{dim}_{\mathbb{Q}_{l}} \operatorname{Im}^{0} \rho_{i}^{(k)}-\operatorname{dim}_{\mathbb{Q}_{l}} \operatorname{Im}^{1} \tau_{i}^{(k+1)}=-\left(\operatorname{dim}_{\mathbb{Q}_{l}} \operatorname{Im}^{1} \rho_{i}^{(k)}-\operatorname{dim}_{\mathbb{Q}_{l}} \operatorname{Im}^{0} \tau_{i-2}^{(k+1)}\right) .
$$

On the other hand, we have $\operatorname{dim}_{\mathbb{Q}_{l}} \operatorname{Im}^{0} \rho_{i}^{(k)}=\operatorname{dim}_{\mathbb{Q}_{l}} \operatorname{Im}^{0} \rho_{2(n-k)-i}^{(k)}$ by Lemma [5.9], and $\operatorname{dim}_{\mathbb{Q}_{l}} \operatorname{Im}^{1} \rho_{i}^{(k)}=\operatorname{dim}_{\mathbb{Q}_{l}} \operatorname{Im}^{1} \rho_{2(n-k)-i+2}^{(k)}$ by Lemma 5.10. Hence, by (15.4), we have

$$
\begin{aligned}
\operatorname{dim}_{\mathbb{Q}_{l}} \operatorname{Im}^{0} \rho_{2(n-k)-i}^{(k)}+\operatorname{dim}_{\mathbb{Q}_{l}} \operatorname{Im}^{1} \rho_{2(n-k)-i+2}^{(k)} \\
=\operatorname{dim}_{\mathbb{Q}_{l}} \operatorname{Im}^{0} \tau_{2(n-k)-i}^{(k+1)}+\operatorname{dim}_{\mathbb{Q}_{l}} \operatorname{Im}^{1} \tau_{2(n-k)-i}^{(k+1)} .
\end{aligned}
$$

From this, by replacing $2(n-k)-i$ by $i$, we have

$$
\operatorname{dim}_{\mathbb{Q}_{l}} \operatorname{Im}^{0} \rho_{i}^{(k)}-\operatorname{dim}_{\mathbb{Q}_{l}} \operatorname{Im}^{1} \tau_{i}^{(k+1)}=-\left(\operatorname{dim}_{\mathbb{Q}_{l}} \operatorname{Im}^{1} \rho_{i+2}^{(k)}-\operatorname{dim}_{\mathbb{Q}_{l}} \operatorname{Im}^{0} \tau_{i}^{(k+1)}\right) .
$$

By combining (5.5) and (5.6), we have

$$
\operatorname{dim}_{\mathbb{Q}_{l}} \operatorname{Im}^{1} \rho_{i}^{(k)}-\operatorname{dim}_{\mathbb{Q}_{l}} \operatorname{Im}^{0} \tau_{i-2}^{(k+1)}=\operatorname{dim}_{\mathbb{Q}_{l}} \operatorname{Im}^{1} \rho_{i+2}^{(k)}-\operatorname{dim}_{\mathbb{Q}_{l}} \operatorname{Im}^{0} \tau_{i}^{(k+1)} .
$$

Hence the difference $\operatorname{dim}_{\mathbb{Q}_{l}} \operatorname{Im}^{1} \rho_{i+2}^{(k)}-\operatorname{dim}_{\mathbb{Q}_{l}} \operatorname{Im}^{0} \tau_{i}^{(k+1)}$ is independent of $i$. Since this is zero for sufficiently large (or small) $i$, we have

$$
\operatorname{dim}_{\mathbb{Q}_{l}} \operatorname{Im}^{1} \rho_{i+2}^{(k)}-\operatorname{dim}_{\mathbb{Q}_{l}} \operatorname{Im}^{0} \tau_{i}^{(k+1)}=0 \quad \text { for all } i
$$


which proves the second assertion. Hence we also have the first assertion by (5.5) or (5.6).

We call the pairing on $H_{\text {ét }}^{i}\left(X^{(k)}\right)$ defined by

$$
H_{\text {ét }}^{i}\left(X^{(k)}\right) \times H_{\text {èt }}^{i}\left(X^{(k)}\right) \rightarrow \mathbb{Q}_{l}, \quad(a, b) \mapsto L^{n-k+1-i} a \cup b
$$

the sum of the Lefschetz pairings. We denote it simply by $\langle$,$\rangle . The pairing \langle$, is nondegenerate by the hard Lefschetz theorem (\$2.2). However, the following lemma is far from trivial, and we use the assumptions of Proposition 5.7 to prove it.

Lemma 5.13. The restriction of $\langle$,$\rangle on H_{\text {ét }}^{i}\left(X^{(k+1)}\right)$ to $\operatorname{Im}^{0} \rho_{i}^{(k)}$ is nondegenerate. Similarly, The restriction of $\langle$,$\rangle on H_{\text {ét }}^{i+2}\left(X^{(k)}\right)$ to $\operatorname{Im}^{0} \tau_{i}^{(k+1)}$ is also nondegenerate.

Proof of Lemma 5.13. Since the decomposition of $\operatorname{Im}^{0} \rho_{i}^{(k)}$ in Lemma [5.9] is orthogonal with respect to $\langle$,$\rangle , it is enough to show that the restriction of \langle$,$\rangle to$ $L^{j} P^{i-2 j} \operatorname{Im}^{0} \rho_{i}^{(k)}$ is nondegenerate for each $j$. By the assumptions of Proposition 5.7. we observe that all cohomology groups of $X^{(k)}, X^{(k+1)}$, maps $\rho, \tau, L$, and pairings $\langle$,$\rangle have natural \mathbb{Q}$-structures (see \$2.1). In particular, the inclusion

$$
L^{j} P^{i-2 j} \operatorname{Im}^{0} \rho_{i}^{(k)} \subset L^{j} P_{\text {ét }}^{i-2 j}\left(X^{(k+1)}\right)
$$

has a $\mathbb{Q}$-structure. Namely, let $V \subset L^{j} P^{i-2 j} \operatorname{Im}^{0} \rho_{i}^{(k)}$ and $W \subset L^{j} P_{\text {ét }}^{i-2 j}\left(X^{(k+1)}\right)$ be the $\mathbb{Q}$-vector subspaces generated by algebraic cycles, then we have

$$
V \otimes_{\mathbb{Q}} \mathbb{Q}_{l}=L^{j} P^{i-2 j} \operatorname{Im}^{0} \rho_{i}^{(k)}, \quad W \otimes_{\mathbb{Q}} \mathbb{Q}_{l}=L^{j} P_{\text {ét }}^{i-2 j}\left(X^{(k+1)}\right) .
$$

It is enough to show that the restriction of $\langle$,$\rangle to V$ is nondegenerate. We may assume $i$ is even because there is no odd degree cohomology. By assumption, the Hodge standard conjecture holds for all irreducible components of $X^{(k+1)}$. Hence the restriction of $\langle$,$\rangle to W$ is positive (resp. negative) definite if $(i-2 j) / 2$ is even (resp. odd). Therefore, the restriction of $\langle$,$\rangle to V$ is nondegenerate because it is definite.

Lemma 5.14. The composition of the following maps is an isomorphism:

$$
\operatorname{Im}^{0} \rho_{i}^{(k)} \stackrel{\tau_{i}^{(k+1)}}{\longrightarrow} \operatorname{Im} \tau_{i}^{(k+1)} \longrightarrow \operatorname{Im}^{1} \tau_{i}^{(k+1)}=\operatorname{Im} \tau_{i}^{(k+1)} / \operatorname{Im}^{0} \tau_{i}^{(k+1)}
$$

Similarly, the composition of the following maps is also an isomorphism:

$$
\operatorname{Im}^{0} \tau_{i}^{(k+1)} \stackrel{\rho_{i+2}^{(k)}}{\longrightarrow} \operatorname{Im} \rho_{i+2}^{(k)} \longrightarrow \operatorname{Im}^{1} \rho_{i+2}^{(k)}=\operatorname{Im} \rho_{i+2}^{(k)} / \operatorname{Im}^{0} \rho_{i+2}^{(k)} .
$$

Proof of Lemma 5.14. By Lemma 5.12, we have only to show that the compositions are injective. Assume that the composition of the first row is not injective and take a nonzero $x \in \operatorname{Im}^{0} \rho_{i}^{(k)}$ such that $\tau_{i}^{(k+1)}(x) \in \operatorname{Im}^{0} \tau_{i}^{(k+1)}$. If $\tau_{i}^{(k+1)}(x) \neq 0$, by Lemma 5.13, there exists $\tau_{i}^{(k+1)}(y) \in \operatorname{Im}^{0} \tau_{i}^{(k+1)}$ such that

$$
L^{2(n-k+1)-(i+2)} \tau_{i}^{(k+1)}(x) \cup \tau_{i}^{(k+1)}(y) \neq 0 .
$$


However, since $x=\rho_{i}^{(k)}\left(x^{\prime}\right)$ for some $x^{\prime} \in H_{\text {ét }}^{i}\left(X^{(k)}\right)$, by (5.1), we have

$$
\begin{aligned}
0 & \neq L^{(n-k+1)-(i+2)} \tau_{i}^{(k+1)}(x) \cup \tau_{i}^{(k+1)}(y) \\
& = \pm L^{(n-k+1)-(i+2)} \rho_{i+2}^{(k)} \circ \tau_{i}^{(k+1)} \circ \rho_{i}^{(k)}\left(x^{\prime}\right) \cup y \\
& =0 .
\end{aligned}
$$

Recall that $\rho_{i+2}^{(k)} \circ \tau_{i}^{(k+1)} \circ \rho_{i}^{(k)}=0$. This is a contradiction. Hence we have $\tau_{i}^{(k+1)}(x)=0$. By Lemma 5.13 again, there exists $\rho_{i}^{(k)}(z) \in \operatorname{Im}^{0} \rho_{i}^{(k)}$ such that

$$
L^{n-k-i} x \cup \rho_{i}^{(k)}(z) \neq 0 .
$$

However, by (5.1), we have

$$
0 \neq L^{n-k-i} x \cup \rho_{i}^{(k)}(z)= \pm L^{n-k-i} \tau_{i}^{(k+1)}(x) \cup z=0
$$

since $\tau_{i}^{(k+1)}(x)=0$. This is a contradiction. Hence the composition of the first row is injective. Similarly, we see that the composition of the second row is also injective.

Lemma 5.15. By Lemma 5.14, the surjections $\operatorname{Im} \tau_{i}^{(k+1)} \rightarrow \operatorname{Im}^{1} \tau_{i}^{(k+1)}$ and $\operatorname{Im} \rho_{i+2}^{(k)} \rightarrow$ $\operatorname{Im}^{1} \rho_{i+2}^{(k)}$ have canonical splittings. Therefore, we have the following decompositions of $\operatorname{Im} \tau_{i}^{(k+1)}$ and $\operatorname{Im} \rho_{i+2}^{(k)}$ :

$$
\operatorname{Im} \tau_{i}^{(k+1)}=\operatorname{Im}^{0} \tau_{i}^{(k+1)} \oplus \operatorname{Im}^{1} \tau_{i}^{(k+1)}, \quad \operatorname{Im} \rho_{i+2}^{(k)}=\operatorname{Im}^{0} \rho_{i+2}^{(k)} \oplus \operatorname{Im}^{1} \rho_{i+2}^{(k)} .
$$

These are orthogonal decompositions with respect to $\langle$,$\rangle .$

Proof of Lemma 5.15. The proof is easy and immediate. Take $(a, b) \in \operatorname{Im}^{0} \tau_{i}^{(k+1)} \oplus$ $\operatorname{Im}^{1} \tau_{i}^{(k+1)}$. Then, by the first assertion of Lemma 5.14 we can write $a=\tau_{i}^{(k+1)}\left(a^{\prime}\right)$ and $b=\tau_{i}^{(k+1)} \circ \rho_{i}^{(k)}\left(b^{\prime}\right)$ for some $a^{\prime}, b^{\prime}$. Then, by (5.1), we have

$$
\begin{aligned}
L^{n-k+1-(i+2)} a \cup b & =L^{n-k+1-(i+2)} \tau_{i}^{(k+1)}\left(a^{\prime}\right) \cup\left(\tau_{i}^{(k+1)} \circ \rho_{i}^{(k)}\left(b^{\prime}\right)\right) \\
& = \pm L^{n-k+1-(i+2)} a^{\prime} \cup\left(\rho_{i+2}^{(k+1)} \circ \tau_{i}^{(k+1)} \circ \rho_{i}^{(k)}\left(b^{\prime}\right)\right) \\
& =0
\end{aligned}
$$

since $\rho_{i+2}^{(k+1)} \circ \tau_{i}^{(k+1)} \circ \rho_{i}^{(k)}=0$. Similarly, we see that the decomposition $\operatorname{Im} \rho_{i+2}^{(k)}=$ $\operatorname{Im}^{0} \rho_{i+2}^{(k)} \oplus \operatorname{Im}^{1} \rho_{i+2}^{(k)}$ is also orthogonal with respect to $\langle$,$\rangle .$

Finally, by combining above results, we shall prove Lemma 5.8 .

Proof of Lemma 5.8. Firstly, we shall prove the first assertion. Since $\tau_{i}^{(k+1)} \circ \rho_{i}^{(k)} \circ$ $\tau_{i-2}^{(k+1)}=0$, the inclusion

$$
\left(\operatorname{Ker} \tau_{i}^{(k+1)}\right) \cap\left(\operatorname{Im} \rho_{i}^{(k)}\right) \supset \operatorname{Im}\left(\rho_{i}^{(k)} \circ \tau_{i-2}^{(k+1)}\right)
$$


is obvious. To prove the opposite inclusion, we take $x \in\left(\operatorname{Ker} \tau_{i}^{(k+1)}\right) \cap\left(\operatorname{Im} \rho_{i}^{(k)}\right)$. Then, for all $y \in H_{\text {ett }}^{i}\left(X^{(k)}\right)$, by (5.1), we have

$$
L^{n-k-i} x \cup \rho_{i}^{(k)}(y)= \pm L^{n-k-i} \tau_{i}^{(k+1)}(x) \cup y=0
$$

since $\tau_{i}^{(k+1)}(x)=0$. By Lemma 5.15 we write $x=x_{0}+x_{1}$ for $x_{0} \in \operatorname{Im}^{0} \rho_{i}^{(k)}$ and $x_{1} \in \operatorname{Im}^{1} \rho_{i}^{(k)}$. If $x_{0} \neq 0$, by Lemma 5.13 and Lemma 5.15, there exists $y^{\prime} \in \operatorname{Im}^{0} \rho_{i}^{(k)}$ such that

$$
\begin{aligned}
L^{n-k-i} x \cup y^{\prime} & =L^{n-k-i} x_{0} \cup y^{\prime}+L^{n-k-i} x_{1} \cup y^{\prime} \\
& =L^{n-k-i} x_{0} \cup y^{\prime} \\
& \neq 0,
\end{aligned}
$$

which is absurd. Hence $x_{0}=0$. Then, we have

$$
x=x_{1} \in \operatorname{Im}^{1} \rho_{i}^{(k)}=\rho_{i}^{(k)}\left(\operatorname{Im}^{0} \tau_{i-2}^{(k+1)}\right) \subset \operatorname{Im}\left(\rho_{i}^{(k)} \circ \tau_{i-2}^{(k+1)}\right) .
$$

This proves the first assertion. Similarly, we can prove the second assertion by using Lemma 5.13 and Lemma 5.15. Therefore, Lemma 5.8 is proved, and the proof of Proposition 5.7 is complete.

Remark 5.16. In the above proof, Lemma 5.13 is the only part where we crucially use the assumptions of Proposition 5.7. In SaM1, M. Saito proved a corresponding statement by using polarized Hodge structures.

\section{Application to p-ADICALly UNIFORMized VARIETIES}

In this section, we give a proof of the main theorem (Theorem 1.2) of this paper. We also give some immediate applications.

6.1. Drinfeld upper half spaces and $p$-adic uniformization. Let $K$ be a complete discrete valuation field with finite residue field $\mathbb{F}_{q}$ of characteristic $p>0$. Let $\widehat{\Omega}_{K}^{d}$ be the Drinfeld upper half space of dimension $d \geq 1$ over $K$, which is a rigid analytic space obtained by removing all $K$-rational hyperplanes from $\mathbb{P}_{K}^{d}$. We have a natural action of $\mathrm{PGL}_{d+1}(K)$ on $\widehat{\Omega}_{K}^{d}$.

As a formal scheme, $\widehat{\Omega}_{K}^{d}$ can be constructed as follows. Take the projective space $\mathbb{P}_{\mathscr{O}_{K}}^{d}$ over $\mathscr{O}_{K}$. Then, take successive blowing-ups of $\mathbb{P}_{\mathscr{O}_{K}}^{d}$ along all linear subvarieties in the special fiber $\mathbb{P}_{\mathbb{F}_{q}}^{d}$ as in 4.1. By continuing this process for all exceptional divisors appearing in the blowing-ups, we obtain a formal scheme $\widehat{\Omega}_{\mathscr{O}_{K}}^{d}$ locally of finite type over $\operatorname{Spf} \mathscr{O}_{K}$. By construction, the rigid analytic space associated with $\widehat{\Omega}_{\mathscr{O}_{K}}^{d}$ is isomorphic to $\widehat{\Omega}_{K}^{d}$. In other words, $\widehat{\Omega}_{K}^{d}$ is the "generic fiber" in the sense of Raynaud ( $\mathrm{Ray}$ ) of the formal scheme $\widehat{\Omega}_{\mathscr{O}_{K}}^{d}$ over $\operatorname{Spf} \mathscr{O}_{K}$.

Let $\Gamma$ be a cocompact torsion free discrete subgroup of $\mathrm{PGL}_{d+1}(K)$. We have a natural action of $\Gamma$ on $\widehat{\Omega}_{\mathscr{O}_{K}}^{d}$, and this action is discontinuous with respect to 
Zariski topology. Then, we take a quotient $\widehat{\mathfrak{X}}_{\Gamma}:=\Gamma \backslash \widehat{\Omega}_{\mathscr{O}_{K}}^{d}$ as a formal scheme. Mustafin, Kurihara showed that the relative dualizing sheaf $\omega_{\widehat{\mathfrak{x}}_{\Gamma} / \mathscr{O}_{K}}$ is invertible and ample. Therefore, $\widehat{\mathfrak{X}}_{\Gamma}$ can be algebraized to a projective scheme $\mathfrak{X}_{\Gamma}$ over $\mathscr{O}_{K}$. The generic fiber $X_{\Gamma}:=\mathfrak{X}_{\Gamma} \otimes_{\mathscr{O}_{K}} K$ is a projective smooth variety over $K$ whose associated rigid analytic space is the rigid analytic quotient $\Gamma \backslash \widehat{\Omega}_{K}^{d}$. By construction, $\mathfrak{X}_{\Gamma}$ is a proper semistable model of $X_{\Gamma}$ over $\mathscr{O}_{K}$ whose special fiber is described by the cell complex $\Gamma \backslash \mathfrak{T}$, where $\mathfrak{T}$ denotes the Bruhat-Tits building of $\mathrm{PGL}_{d+1}(K)$ (for details, see [Mus, Theorem 4.1, [Ku], Theorem 2.2.5).

6.2. Proof of the main theorem (Theorem 1.2). As in $\$ 6.1$ let $K$ be a complete discrete valuation field with finite residue field $\mathbb{F}_{q}$ of characteristic $p>0$, $\Gamma \subset \mathrm{PGL}_{d+1}(K)$ a cocompact torsion free discrete subgroup, and $\mathfrak{X}_{\Gamma}$ an algebraization of $\Gamma \backslash \widehat{\Omega}_{\mathscr{O}_{K}}^{d}$ with generic fiber $X_{\Gamma}$ as in 86.1$]$

The following lemma seems well-known.

Lemma 6.1. Let $X, Y$ be proper smooth variety of dimension $d$ over $K$, and $f: X \rightarrow Y$ a proper surjective generically finite morphism of degree $\operatorname{deg} f$ ( $f$ is called an alteration in $\mathrm{dJ}$ ). If the weight-monodromy conjecture (Conjecture 1.1) holds for $X$, then Conjecture 1.1 holds also for $Y$.

Proof. Since $f$ is finite of degree $\operatorname{deg} f$ on some open dense subscheme $U \subset$ $X, f^{*}: H_{\text {êt }}^{k}\left(Y_{\bar{K}}, \mathbb{Q}_{l}\right) \rightarrow H_{\text {êt }}^{k}\left(X_{\bar{K}}, \mathbb{Q}_{l}\right)$ and $f_{*}: H_{\text {êt }}^{k}\left(X_{\bar{K}}, \mathbb{Q}_{l}\right) \rightarrow H_{\text {êt }}^{k}\left(Y_{\bar{K}}, \mathbb{Q}_{l}\right)$ satisfy $f_{*} \circ f^{*}=$ multiplication by $\operatorname{deg} f$. Since $f_{*}, f^{*}$ commute with $\operatorname{Gal}(\bar{K} / K)$-action, $H_{\mathrm{ett}}^{k}\left(Y_{\bar{K}}, \mathbb{Q}_{l}\right)$ is a direct summand of $H_{\mathrm{èt}}^{k}\left(X_{\bar{K}}, \mathbb{Q}_{l}\right)$ as a $\mathrm{Gal}(\bar{K} / K)$-representation. Therefore, the restriction of the monodromy filtration (resp. weight filtration) on $H_{\text {êt }}^{k}\left(X_{\bar{K}}, \mathbb{Q}_{l}\right)$ to $H_{\text {êt }}^{k}\left(Y_{\bar{K}}, \mathbb{Q}_{l}\right)$ coincides with the monodromy filtration (resp. weight filtration) on $H_{\text {ét }}^{k}\left(Y_{\bar{K}}, \mathbb{Q}_{l}\right)$ itself. Hence the assertion follows.

Now, we shall prove the main theorem (Theorem 1.2) of this paper.

Proof of Theorem 1.2. First of all, it is known that the intersection of all finite index subgroups $\Gamma^{\prime} \subset \Gamma$ is equal to the identity (Ga, Theorem 2.7, Proof, (b)). From this, by looking at the action of $\Gamma$ on the Bruhat-Tits building $\mathfrak{T}$ of $\mathrm{PGL}_{d+1}(K)$ ( $\left[\mathrm{Mus},[\mathrm{Ku}]\right.$ ), it is easy to see that $\mathfrak{X}_{\Gamma^{\prime}}$ is strictly semistable for some finite index subgroup $\Gamma^{\prime} \subset \Gamma$. Since we have a finite étale covering $X_{\Gamma^{\prime}} \rightarrow X_{\Gamma}$, by Lemma 6.1, we may assume that $\mathfrak{X}_{\Gamma}$ is a strictly semistable model of $X_{\Gamma}$ over $\mathscr{O}_{K}$.

Under this assumption, from the construction of $\mathfrak{X}_{\Gamma}$ in 6.1 , we see that all irreducible components $X_{1}, \ldots, X_{m}$ of the special fiber of $\mathfrak{X}_{\Gamma}$ are isomorphic to the variety $B^{d}$ constructed in 4.1 By Proposition 4.2. 4, 5, for $i \neq j$, each irreducible component of $X_{i} \cap X_{j}$ is isomorphic to a divisor of the form $D_{V}$ on $B^{d}$ in 84.2 Moreover, by induction, we see that, for $1 \leq i_{1}<\cdots<i_{k} \leq m$, each 
irreducible component $Y$ of $X_{i_{1}} \cap \cdots \cap X_{i_{k}}$ is isomorphic to the product

$$
Y \cong B^{n_{1}} \times \cdots \times B^{n_{k}}
$$

for some $n_{1}, \ldots, n_{k} \geq 1$ with $n_{1}+\cdots+n_{k}=d-k+1$.

Let $\mathscr{L}$ be the relative dualizing sheaf $\omega_{\mathfrak{X}_{\Gamma} / \mathscr{O}_{K}}$, which is invertible and ample by Mustafin, Kurihara ([Mus, $\mathrm{Ku}$ ). Take $i$ with $1 \leq i \leq m$ and fix an isomorphism $X_{i} \cong B^{d}$. Then, by an explicit calculation in [Mus, $[\mathrm{Ku}$, the restriction of $\mathscr{L}$ to each $X_{i}$ is isomorphic to

$$
-(n+1) f^{*} \mathscr{O}_{\mathbb{P} d}(1)+\sum_{d=0}^{n-1}(n-d) D_{d}
$$

(for notation, see 44.1, s.2, see also Remark 4.8), which is an ample $\mathrm{PGL}_{d+1}\left(\mathbb{F}_{q}\right)$ invariant divisor on $X_{i} \cong B^{d}$. Therefore, by Proposition 4.7 the Hodge standard conjecture (Conjecture 2.6) holds for $\left(X_{i},\left.\mathscr{L}\right|_{X_{i}}\right)$.

Moreover, for $1 \leq i_{1}<\cdots<i_{k} \leq m$, take an irreducible component $Y$ of $X_{i_{1}} \cap \cdots \cap X_{i_{k}}$. Then, $Y$ is isomorphic to $B^{n_{1}} \times \cdots \times B^{n_{k}}$ for some $n_{1}, \ldots, n_{k} \geq 1$ with $n_{1}+\cdots+n_{k}=d-k+1$. By the construction of $B^{n}$ in 4.1 and the Künneth formula, $Y$ satisfies Assumption 2.1 in $\$ 2.1$ (see also $\$ 2.5$ ). Let $\operatorname{pr}_{j}: Y \rightarrow B^{n_{j}}$ be the projection to the $i$-th factor for $j=1, \ldots, k$. Then, by applying Proposition 4.2, 4, 5 and Proposition 4.4, 4 inductively, we see that the restriction of $\mathscr{L}$ to $Y$ is of the form

$$
\left.\mathscr{L}\right|_{Y}=\operatorname{pr}_{1}^{*} \mathscr{O}\left(L_{1}\right)+\cdots+\operatorname{pr}_{k}^{*} \mathscr{O}\left(L_{k}\right) \quad \text { in } \quad H^{2}(Y)
$$

where $L_{j}$ is an ample $\mathrm{PGL}_{n_{j}+1}\left(\mathbb{F}_{q}\right)$-invariant divisor on $B^{n_{j}}$ for $j=1, \ldots, k$. By Proposition 4.7. the Hodge standard conjecture (Conjecture 2.6) holds for $\left(B^{n_{j}}, L_{j}\right)$ for $j=1, \ldots, k$. By applying Proposition 2.13 inductively, we conclude that the Hodge standard conjecture (Conjecture 2.6) also holds for $\left(Y,\left.\mathscr{L}\right|_{Y}\right)$.

Therefore, $\mathfrak{X}_{\Gamma}$ satisfies all assumptions in Proposition [5.7. Hence the weightmonodromy conjecture (Conjecture 1.1) holds for $X_{\Gamma}$, and the proof of Theorem 1.2 is complete.

Remark 6.2. Theorem 1.2 is new only in mixed characteristic. However, we note that Theorem 1.2 in characteristic $p>0$ does not automatically follow from Deligne's results in De3 because $\mathfrak{X}_{\Gamma}$ does not automatically come from a family of varieties over a curve over a finite field (This was explained by Illusie in [I13, 8.7). We need a specialization argument to reduce the general characteristic $p>0$ case to Deligne's case ([Te, [t1] ).

6.3. Schneider-Stuhler's conjecture on the filtration $F^{\bullet}$. In [SS], SchneiderStuhler computed the cohomology of $\widehat{\Omega}_{K}^{d}$ and its quotient $\Gamma \backslash \widehat{\Omega}_{K}^{d}$ by methods from rigid analytic geometry and representation theory. Their results are valid for cohomology theories satisfying certain axioms, and they showed that de Rham cohomology and rigid analytic $l$-adic cohomology satisfy these axioms. Precisely speaking, at that time, they used unpublished results of Gabber to check the 
axioms for rigid analytic $l$-adic cohomology. After that, these axioms were es-

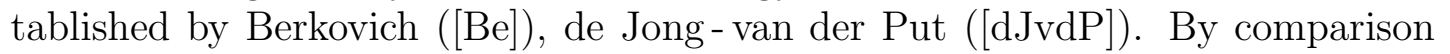
theorem between rigid analytic and usual l-adic cohomology, Schneider-Stuhler's computation is valid for $l$-adic cohomology of the variety $X_{\Gamma}$. Here we combine our results with the results of Schneider-Stuhler in [SS], and prove SchneiderStuhler's conjecture on the filtration $F^{\bullet}$ for $l$-adic cohomology.

First of all, we recall the results of Schneider-Stuhler (for details, see [SS], Theorem 4). As in $\$ 6.1$ let $K$ be a complete discrete valuation field with finite residue field $\mathbb{F}_{q}$ of characteristic $p>0$. For a cocompact torsion free discrete subgroup $\Gamma \subset \mathrm{PGL}_{d+1}(K)$, let

$$
\operatorname{Ind}_{\Gamma}:=C^{\infty}\left(\operatorname{PGL}_{d+1}(K) / \Gamma, \mathbb{C}\right)
$$

be the $\mathrm{PGL}_{d+1}(K)$-representation induced from the trivial character on $\Gamma$. Let $\mu(\Gamma)$ be the multiplicity of the Steinberg representation in $\operatorname{Ind}_{\Gamma}$. In $[\mathrm{SS}$, $\S 5$, Schneider-Stuhler explicitly computed the $E_{2}$-terms of a Hochschild-Serre type covering spectral sequence:

$$
E_{2}^{r, s}=H^{r}\left(\Gamma, H_{\text {ét }}^{s}\left(\widehat{\Omega}_{K}^{d} \otimes_{K} \bar{K}, \mathbb{Q}_{l}\right)\right) \Longrightarrow H_{\text {ét }}^{r+s}\left(X_{\Gamma} \otimes_{K} \bar{K}, \mathbb{Q}_{l}\right)
$$

([SS], §5, Proposition 2), and proved

$$
H_{\text {ét }}^{k}\left(X_{\Gamma} \otimes_{K} \bar{K}, \mathbb{Q}_{l}\right) \cong \begin{cases}\mathbb{Q}_{l}\left(-\frac{k}{2}\right) & \text { if } k \text { is even, } 0 \leq k \leq 2 d, k \neq d \\ 0 & \text { if } k \text { is odd, } k \neq d .\end{cases}
$$

For the middle degree $k=d$, they proved that the covering spectral sequence (6.1) defines a decreasing filtration $F^{\bullet}$ on $V:=H_{\text {ét }}^{d}\left(X_{\Gamma} \otimes_{K} \bar{K}, \mathbb{Q}_{l}\right)$ such that

$$
\begin{gathered}
V=F^{0} V \supset F^{1} V \supset \cdots \supset F^{d} V \supset F^{d+1} V=0, \\
F^{r} V / F^{r+1} V \cong \begin{cases}\mathbb{Q}_{l}(r-d)^{\oplus \mu(\Gamma)} & \text { if } 0 \leq r \leq d, r \neq \frac{d}{2} \\
\mathbb{Q}_{l}\left(-\frac{d}{2}\right)^{\oplus(\mu(\Gamma)+1)} & \text { if } r=\frac{d}{2} \\
0 & \text { otherwise, }\end{cases}
\end{gathered}
$$

and conjectured that $F^{\bullet}$ essentially coincides with the monodromy filtration (see [SS], introduction and a remark following Theorem 5).

Theorem 6.3 (Schneider-Stuhler's conjecture for l-adic cohomology). Let $M_{\bullet}$ be the monodromy filtration on $V$ (Definition 5.1). Define an increasing filtration $F_{\text {. }}^{\prime}$ on $V$ by $F_{i}^{\prime} V=F^{-\lfloor i / 2\rfloor} V$. Then, we have

$$
M_{i} V=F_{i-d}^{\prime} V \quad \text { for all } i \text {. }
$$

Proof. We compute the graded quotients of $F_{\bullet}^{\prime}$ explicitly. If $i$ is even, we have

$$
\begin{aligned}
F_{i}^{\prime} V / F_{i-1}^{\prime} V & =F^{-i / 2} V / F^{-i / 2+1} V \\
& = \begin{cases}\mathbb{Q}_{l}\left(-\frac{i}{2}-d\right)^{\oplus \mu(\Gamma)} & \text { if }-2 d \leq i \leq 0, i \neq-d \\
\mathbb{Q}_{l}\left(-\frac{d}{2}\right)^{\oplus(\mu(\Gamma)+1)} & \text { if } i=-d \\
0 & \text { otherwise. }\end{cases}
\end{aligned}
$$


If $i$ is odd, we have $\lfloor i / 2\rfloor=\lfloor(i-1) / 2\rfloor$ hence $F_{i}^{\prime} V / F_{i-1}^{\prime} V=0$. Therefore, each $F_{i}^{\prime} V / F_{i-1}^{\prime} V$ has weight $i+2 d$ as a $\operatorname{Gal}\left(\overline{\mathbb{F}}_{q} / \mathbb{F}_{q}\right)$-representation. Now the assertion follows from Theorem 1.2 .

6.4. Application to the local zeta function of $X_{\Gamma}$. Let notation be as in $\$ 6.3$, Let us recall the definition of the local zeta functions (for details, see [Se2, Rap). For a continuous $\operatorname{Gal}(\bar{K} / K)$-representation $V$ over $\mathbb{Q}_{l}$, the local L-function of $V$ is defined by

$$
L(s, V):=\operatorname{det}\left(1-q^{-s} \cdot \operatorname{Fr}_{q} ; V^{I_{K}}\right)^{-1}
$$

(for notation, see $\$ 5.1, \$ 5.2)$. For a variety $X$ over $K$, the local zeta function of $X$ is defined by

$$
\zeta(s, X):=\prod_{k=0}^{2 \operatorname{dim} X} L\left(s, H_{\text {ét }}^{k}\left(X_{\bar{K}}, \mathbb{Q}_{l}\right)\right)^{(-1)^{k}} .
$$

Let us compute the local zeta function of $X_{\Gamma}$. For an even integer $k$ with $0 \leq k \leq 2 d, k \neq d$, by (6.2), we easily see that

$$
L\left(s, H_{\text {ét }}^{k}\left(X_{\Gamma} \otimes_{K} \bar{K}, \mathbb{Q}_{l}\right)\right)=\operatorname{det}\left(1-q^{-s} \cdot \operatorname{Fr}_{q} ; \mathbb{Q}_{l}\left(-\frac{k}{2}\right)\right)^{-1}=\frac{1}{1-q^{k / 2-s}} .
$$

On the other hand, for the middle degree $k=d$, we can not compute the local $L$ function only from the results of Schneider-Stuhler (6.3) because (6.3) only gives us the semisimplification of the $\operatorname{Gal}(\bar{K} / K)$-representation $H_{\text {ét }}^{d}\left(X_{\Gamma} \otimes_{K} \bar{K}, \mathbb{Q}_{l}\right)$. We need the weight-monodromy conjecture to recover the local $L$-function from the semisimplification (see Rap, $\S 2$ ).

Now, we shall use Theorem 6.3. By Theorem 6.3, we see that the inertia fixed part is given by

$$
H_{\text {ét }}^{d}\left(X_{\Gamma} \otimes_{K} \bar{K}, \mathbb{Q}_{l}\right)^{I_{K}}= \begin{cases}\mathbb{Q}_{l}^{\oplus \mu(\Gamma)} \oplus \mathbb{Q}_{l}\left(-\frac{d}{2}\right) & \text { if } d \text { is even } \\ \mathbb{Q}_{l}^{\oplus \mu(\Gamma)} & \text { if } d \text { is odd }\end{cases}
$$

(see also Definition 5.1, 3). Therefore, we have

$$
L\left(s, H_{\text {ét }}^{d}\left(X_{\Gamma} \otimes_{K} \bar{K}, \mathbb{Q}_{l}\right)\right)= \begin{cases}\frac{1}{\left(1-q^{-s}\right)^{\mu(\Gamma)}} \cdot \frac{1}{1-q^{d / 2-s}} & \text { if } d \text { is even } \\ \frac{1}{\left(1-q^{-s}\right)^{\mu(\Gamma)}} & \text { if } d \text { is odd } .\end{cases}
$$

By combining above results, we have the following theorem.

Theorem 6.4 (Local zeta function of $\left.X_{\Gamma}\right)$. Let $\Gamma \subset \mathrm{PGL}_{d+1}(K)$ be a cocompact torsion free discrete subgroup. Then, the local zeta function of $X_{\Gamma}$ is given by

$$
\zeta\left(s, X_{\Gamma}\right)=\left(1-q^{-s}\right)^{\mu(\Gamma) \cdot(-1)^{d+1}} \cdot \prod_{k=0}^{d} \frac{1}{1-q^{k-s}},
$$

where $\mu(\Gamma)$ is the multiplicity of the Steinberg representation in $\operatorname{Ind}_{\Gamma}$ as in 6.3 . 
Remark 6.5. As a consequence, we also see that the local zeta function $\zeta\left(s, X_{\Gamma}\right)$ is independent of $l \neq p$. In $\$ 6.5$, we consider the case $l=p$ via $p$-adic Hodge theory.

6.5. $p$-adic weight-monodromy conjecture. We recall some results in $p$-adic Hodge theory to state a $p$-adic analogue of the weight-monodromy conjecture.

Let $K$ be a finite extension of $\mathbb{Q}_{p}$ with residue field $\mathbb{F}_{q}, W\left(\mathbb{F}_{q}\right)$ the ring of Witt vectors with coefficients in $\mathbb{F}_{q}, K_{0}$ the field of fractions of $W\left(\mathbb{F}_{q}\right)$, and $X$ a proper smooth variety over $K$. For simplicity, we assume that there is a proper strictly semistable model $\mathfrak{X}$ of $X$ over $\mathscr{O}_{K}$ (see Definition [5.5).

For $V:=H_{\text {et }}^{w}\left(X_{\bar{K}}, \mathbb{Q}_{p}\right)$, by the $C_{\text {st }}$-conjecture proved by Tsuji, there is a canonical isomorphism

$$
D_{\text {st }}(V):=\left(V \otimes_{\mathbb{Q}_{p}} B_{\text {st }}\right)^{\operatorname{Gal}(\bar{K} / K)} \cong H_{\text {log-crys }}^{w}\left(\mathfrak{X}_{\mathbb{F}_{q}}^{\times} / W\left(\mathbb{F}_{q}\right)^{\times}\right) \otimes_{W\left(\mathbb{F}_{q}\right)} K_{0}
$$

where $B_{\mathrm{st}}$ is Fontaine's ring of $p$-adic periods, $\operatorname{Gal}(\bar{K} / K)$ acts on $V \otimes_{\mathbb{Q}_{p}} B_{\mathrm{st}}$ diagonally, and $H_{\log \text {-crys }}^{w}\left(\mathfrak{X}_{\mathbb{F}_{q}}^{\times} / W\left(\mathbb{F}_{q}\right)^{\times}\right)$is the log crystalline cohomology of the special fiber $\mathfrak{X}_{\mathbb{F}_{q}}=\mathfrak{X} \otimes_{\mathscr{O}_{K}} \mathbb{F}_{q}$ endowed with a natural log structure ([Fo], $\mathrm{HK}$, $[\mathrm{Ka}$, [Ts $)$. It is known that $\operatorname{dim}_{K_{0}} D_{\mathrm{st}}(V)=\operatorname{dim}_{\mathbb{Q}_{p}} V$. Moreover, it is also known that the monodromy operator $N$ and the Frobenius endomorphism $\varphi$ act on both hand sides, and (6.4) is compatible with the actions of them. By using $N, \varphi$, we can define the monodromy filtration $M_{\bullet}$ and the weight filtration $W_{\bullet}$ on $D_{\mathrm{st}}(V)$ by the same way as in Definition 5.1. Definition 5.3. Then, we have the following $p$-adic analogue of the weight-monodromy conjecture.

Conjecture 6.6 ( $p$-adic weight-monodromy conjecture).

$$
M_{i} D_{\mathrm{st}}(V)=W_{i+w} D_{\mathrm{st}}(V) \quad \text { for all } i .
$$

Let notation be as in 5.3 .3 Mokrane constructed a $p$-adic analogue of the weight spectral sequence of Rapoport-Zink as follows:

$$
\begin{aligned}
E_{1}^{-r, w+r}=\bigoplus_{k \geq \max \{0,-r\}} H_{\text {crys }}^{w-r-2 k}\left(X^{(2 k+r+1)} / W\left(\mathbb{F}_{q}\right)\right)(-r-k) \\
\Longrightarrow \quad H_{\text {log-crys }}^{w}\left(\mathfrak{X}_{\mathbb{F}_{q}}^{\times} / W\left(\mathbb{F}_{q}\right)^{\times}\right),
\end{aligned}
$$

where $H_{\text {crys }}^{*}$ denotes the crystalline cohomology ( $[\mathrm{Mo}, \S 3.23$, Théorème 3.32). This spectral sequence has similar properties as the $l$-adic case (compare with \$5.3). By the Weil conjecture for crystalline cohomology ([KM], CLS]), this spectral sequence degenerates at $E_{2}$ modulo torsion, and defines the weight filtration $W_{\bullet}$ on $H_{\log \text {-crys }}^{w}\left(\mathfrak{X}_{\mathbb{F}_{q}}^{\times} / W\left(\mathbb{F}_{q}\right)^{\times}\right)$. Moreover, there is a monodromy operator $N$ satisfying the same properties as the $l$-adic case $(N$ coincides with $\nu$ in $\underline{\mathrm{MO}}$, $\S 3.33)$.

Therefore, by (6.4), Conjecture 6.6] is equivalent to the following conjecture on the weight spectral sequence of Mokrane (compare with Conjecture 5.6). 
Conjecture 6.7 ([M], Conjecture 3.27, §3.33). $N^{r}$ induces an isomorphism

$$
N^{r}: E_{2}^{-r, w+r}(r) \otimes_{W\left(\mathbb{F}_{q}\right)} K_{0} \stackrel{\cong}{\longrightarrow} E_{2}^{r, w-r} \otimes_{W\left(\mathbb{F}_{q}\right)} K_{0}
$$

for all $r, w$.

Theorem 6.8 ( $p$-adic weight-monodromy conjecture for $\left.X_{\Gamma}\right)$. Let $\Gamma \subset \mathrm{PGL}_{d+1}(K)$ be a cocompact torsion free discrete subgroup. Then, a p-adic analogue of the weight-monodromy conjecture (Conjecture 6.6) holds for $X_{\Gamma}$.

Proof. Note that $\mathfrak{X}_{\Gamma}$ is semistable but not necessarily strictly semistable in general. However, as we see in $\$ 6.2, \mathfrak{X}_{\Gamma^{\prime}}$ is strictly semistable for some finite index subgroup $\Gamma^{\prime} \subset \Gamma$. Since a $p$-adic analogue of Lemma 6.1 holds by the same reason, we may assume that $\mathfrak{X}_{\Gamma}$ is strictly semistable. Then, we can prove Theorem 6.8 by the same way as the $l$-adic case. We use the cycle map for crystalline cohomology (GM], Gr $)$.

Remark 6.9. After this work was completed, the author was informed that Ehud de Shalit obtained Theorem 6.8 by a completely different method. His proof relies on a combinatorial result of Alon-de Shalit about harmonic cochains on the Bruhat-Tits buildings ( $\mathrm{AdS}, \mathrm{dS}]$ ).

6.6. Application to the $p$-adic local zeta function of $X_{\Gamma}$. Let notation be as in $\$ 6.5$. Recall that a finite dimensional continuous $\mathrm{Gal}(\bar{K} / K)$-representation $V$ over $\mathbb{Q}_{p}$ is called semistable if $\operatorname{dim}_{K_{0}} D_{\text {st }}(V)=\operatorname{dim}_{\mathbb{Q}_{p}} V([\mathrm{Fo}])$. For semistable $V$, the $p$-adic local L-function of $V$ is defined by

$$
L_{p \text {-adic }}(s, V):=\operatorname{det}\left(1-q^{-s} \cdot \varphi ; D_{\text {st }}(V)^{N=0}\right)^{-1},
$$

where $D_{\text {st }}(V)^{N=0}$ denotes the kernel of $N$ acting on $D_{\text {st }}(V)$. In fact, we can define $L_{p \text {-adic }}(s, V)$ for potentially semistable $V$, but we omit it here (for details, see $[\mathrm{Fo})$. For a variety $X$ over $K$ such that $H_{\text {ét }}^{k}\left(X_{\bar{K}}, \mathbb{Q}_{p}\right)$ are semistable for all $k$, we define the $p$-adic local zeta function $\zeta_{p \text {-adic }}(s, X)$ of $X$ as follows:

$$
\zeta_{p \text {-adic }}(s, X):=\prod_{k=0}^{2 \operatorname{dim} X} L_{p \text {-adic }}\left(s, H_{\text {ét }}^{k}\left(X_{\bar{K}}, \mathbb{Q}_{p}\right)\right)^{(-1)^{i}} .
$$

Here we put the subscript " $p$-adic" to distinguish it from the $l$-adic case.

Theorem 6.10 ( $p$-adic local zeta function of $\left.X_{\Gamma}\right)$. Let $\Gamma \subset \mathrm{PGL}_{d+1}(K)$ be a cocompact torsion free discrete subgroup. Then, for all $k$, the p-adic étale cohomology $H_{\text {ét }}^{k}\left(X_{\Gamma} \otimes_{K} \bar{K}, \mathbb{Q}_{p}\right)$ is a semistable $\operatorname{Gal}(\bar{K} / K)$-representation, and the p-adic local $L$-function $L_{p \text {-adic }}\left(s, H_{\text {ét }}^{k}\left(X_{\Gamma} \otimes_{K} \bar{K}, \mathbb{Q}_{p}\right)\right)$ is the same as the usual local $L$-function defined by l-adic cohomology for $l \neq p$ (see 6.4$)$ :

$$
L_{p \text {-adic }}\left(s, H_{\text {ét }}^{k}\left(X_{\Gamma} \otimes_{K} \bar{K}, \mathbb{Q}_{p}\right)\right)=L\left(s, H_{\text {ét }}^{k}\left(X_{\Gamma} \otimes_{K} \bar{K}, \mathbb{Q}_{l}\right)\right) \text {. }
$$

In particular, we have the following equality of local zeta functions of $X_{\Gamma}$ :

$$
\zeta_{p \text {-adic }}\left(s, X_{\Gamma}\right)=\zeta\left(s, X_{\Gamma}\right)=\left(1-q^{-s}\right)^{\mu(\Gamma) \cdot(-1)^{d+1}} \cdot \prod_{k=0}^{d} \frac{1}{1-q^{k-s}},
$$


where $\mu(\Gamma)$ is the multiplicity of the Steinberg representation in $\operatorname{Ind}_{\Gamma}$ as in 96.3 .

Proof. If $\mathfrak{X}_{\Gamma}$ is strictly semistable, $H_{\mathrm{ett}}^{k}\left(X_{\Gamma} \otimes_{K} \bar{K}, \mathbb{Q}_{p}\right)$ is semistable by the $C_{\mathrm{st}^{-}}$ conjecture proved by Tsuji $(\overline{T s})$. The general case follows from this because $\mathfrak{X}_{\Gamma^{\prime}}$ is strictly semistable for some finite index subgroup $\Gamma^{\prime} \subset \Gamma$ (see also Lemma 6.1 and the beginning of the proof of Theorem 1.2 in $\sqrt{6.2}$.

For the local $L$-functions, we compare the $l$-adic and the $p$-adic case (The following argument is inspired by $[\mathrm{SaT}]$ ). Fix a prime number $l \neq p$. Since the weight-monodromy conjecture holds for both $l$-adic and $p$-adic cohomology (Theorem 1.2. Theorem [6.8), it is enough to prove the equality:

$$
\operatorname{Tr}\left(\operatorname{Fr}_{q}^{m} ; H_{\text {ét }}^{k}\left(X_{\Gamma} \otimes_{K} \bar{K}, \mathbb{Q}_{l}\right)\right)=\operatorname{Tr}\left(\varphi^{m} ; D_{\text {st }}\left(H_{\text {ét }}^{k}\left(X_{\Gamma} \otimes_{K} \bar{K}, \mathbb{Q}_{p}\right)\right)\right)
$$

for all $k, m \geq 0($ Rap , Lemma 2.12). Since

$$
\operatorname{dim}_{\mathbb{Q}_{l}} H_{\text {êt }}^{k}\left(X_{\Gamma} \otimes_{K} \bar{K}, \mathbb{Q}_{l}\right)=\operatorname{dim}_{\mathbb{Q}_{p}} H_{\text {ét }}^{k}\left(X_{\Gamma} \otimes_{K} \bar{K}, \mathbb{Q}_{p}\right),
$$

$H_{\text {êt }}^{k}\left(X_{\Gamma} \otimes_{K} \bar{K}, \mathbb{Q}_{p}\right)=0$ if $k$ is odd and $k \neq d$, and $H_{\text {êt }}^{k}\left(X_{\Gamma} \otimes_{K} \bar{K}, \mathbb{Q}_{p}\left(\frac{k}{2}\right)\right)$ is one dimensional and generated by algebraic cycles if $k$ is even and $k \neq d$. Therefore, (6.5) trivially holds for $k \neq d$. On the other hand, the alternating sum of (6.5) for all $k$ holds by Ochiai's results ( $\underline{0}$, Theorem D). Hence (6.5) holds also for $k=d$.

Remark 6.11. If $\mathfrak{X}_{\Gamma}$ is strictly semistable, we can directly prove Theorem 6.10 without using Ochiai's results as follows. It is easy to see that $E_{1}^{i, j}, d_{1}^{i, j}, N$ of the $l$-adic and the $p$-adic weight spectral sequences have the same $\mathbb{Q}$-structure coming from the groups of algebraic cycles on the special fiber. Hence the characteristic polynomials of $\operatorname{Fr}_{q}, \varphi$ acting on each $E_{2}^{i, j}$ are equal. Since the weight-monodromy conjecture holds for both $l$-adic and $p$-adic cohomology (Theorem 1.2, Theorem 6.8), we see that the characteristic polynomials of $\mathrm{Fr}_{q}, \varphi$ on each graded quotient of the monodromy filtration on $H_{\text {êt }}^{k}\left(X_{\Gamma} \otimes_{K} \bar{K}, \mathbb{Q}_{l}\right), D_{\text {st }}\left(H_{\text {êt }}^{k}\left(X_{\Gamma} \otimes_{K} \bar{K}, \mathbb{Q}_{p}\right)\right)$ are equal for all $k$. Hence we have Theorem 6.10,

Remark 6.12. The proof above is somewhat indirect compared with the $l$-adic case. The reason why we have to make such a detour is that Schneider-Stuhler's results on de Rham cohomology are not enough to compute the $p$-adic local zeta functions because we do not have the action of $\varphi$ on de Rham cohomology in [SS. However, by a recent work of Große-Klönne, we have the action of $\varphi$ on the de Rham cohomology of $\widehat{\Omega}_{K}^{d}$ (GK]). By using his results, it seems possible to compute the $p$-adic local zeta functions directly without using $l$-adic cohomology.

\section{Appendix : Application to the Tate conjecture for varieties ADMITTING $p$-ADIC UNIFORMIZATION}

Here we give an application of the results in the previous section to the Tate conjecture for varieties over number fields admitting $p$-adic uniformization. Such an application was pointed out to the author by Michael Rapoport and the author 
includes it here with his permission. The results in this appendix are essentially due to him.

Let $K$ be a number field, $\bar{K}$ an algebraic closure of $K$, and $X$ a proper smooth scheme over $K$ of dimension $d \geq 1$ which is not necessarily geometrically connected. We say $X$ admits $p$-adic uniformization if there is a finite extension $L$ of $K$ inside $\bar{K}$, and a finite place $w$ of $L$ such that each connected component of $X \otimes_{K} L_{w}$ is isomorphic to $X_{\Gamma}$ for a cocompact torsion free discrete subgroup $\Gamma \subset \mathrm{PGL}_{d+1}\left(L_{w}\right)$, where $L_{w}$ is the completion of $L$ at $w$.

As an application of the results in the previous section, we prove the Tate conjecture for $X$ as follows.

Theorem 7.1 (cf. [Ta, Conjecture 1). Let $X$ be a proper smooth scheme over a number field $K$ of dimension $d \geq 1$ admitting $p$-adic uniformization. Fix a prime number $l$. Then, for any finite extension $K^{\prime}$ of $K$ inside $\bar{K}$ and for any $k, 0 \leq k \leq d$, the $\mathbb{Q}_{l}$-vector space

$$
H_{\text {ét }}^{2 k}\left(X \otimes_{K} \bar{K}, \mathbb{Q}_{l}(k)\right)^{\operatorname{Gal}\left(\bar{K} / K^{\prime}\right)}
$$

is generated by algebraic cycles on $X$ of codimension $k$ defined over $K^{\prime}$.

Remark 7.2. In fact, as we see in the proof below, the above $\mathbb{Q}_{l}$-vector spaces are generated by power of the canonical bundles of connected components of $X \otimes_{K} K^{\prime}$.

Proof. Since it is enough to prove the assertion after replacing $K^{\prime}$ by a finite extension of it, we may assume $K^{\prime}$ contains $L$. Fix a finite place $w^{\prime}$ of $K^{\prime}$ over $w$, and take a connected component $X^{\prime}$ of $X \otimes_{K} K^{\prime}$. Then, $X^{\prime} \otimes_{K^{\prime}} K_{w^{\prime}}^{\prime}$ is isomorphic to $X_{\Gamma} \otimes_{L_{w}} K_{w^{\prime}}^{\prime}$ for some $\Gamma \subset \mathrm{PGL}_{d+1}\left(L_{w}\right)$. We put $V:=H_{\text {ét }}^{2 k}\left(X^{\prime} \otimes_{K^{\prime}} \bar{K}, \mathbb{Q}_{l}(k)\right)$.

We shall prove that $V^{\mathrm{Gal}\left(\bar{K} / K^{\prime}\right)}$ is generated by a power of the canonical bundle $\mathscr{L}$ of $X^{\prime}$. If $k \neq d / 2$, the assertion trivially holds because $\mathscr{L}$ is ample by Mustafin, Kurihara ( $\mathrm{Mus},[\mathrm{Ku}]$ ), and $V$ is one dimensional by Schneider-Stuhler ([SS], see also 6.31 .

If $d$ is even and $k=d / 2$, we shall prove that $V^{\mathrm{Gal}\left(\bar{K} / K^{\prime}\right)}$ is one dimensional. We know that this space has dimension $\geq 1$ because it contains the subspace generated by a power of $\mathscr{L}$. To prove the opposite inequality, we consider the subspace $V^{\operatorname{Gal}\left(\overline{K_{w^{\prime}}^{\prime}} / K_{w^{\prime}}^{\prime}\right)}$ of $V$ consisting of elements fixed by the action of $\operatorname{Gal}\left(\overline{K_{w^{\prime}}^{\prime}} / K_{w^{\prime}}^{\prime}\right)$. Here we fix an embedding $\bar{K} \hookrightarrow \overline{K_{w^{\prime}}^{\prime}}$ over $K^{\prime}$ and consider $\operatorname{Gal}\left(\overline{K_{w^{\prime}}^{\prime}} / K_{w^{\prime}}^{\prime}\right)$ as a subgroup of $\operatorname{Gal}\left(\bar{K} / K^{\prime}\right)$. Since $V^{\mathrm{Gal}\left(\overline{K_{w^{\prime}}^{\prime}} / K_{w^{\prime}}^{\prime}\right)}$ is one dimensional by the following lemma and $V^{\operatorname{Gal}\left(\bar{K} / K^{\prime}\right)} \subset V^{\mathrm{Gal}\left(\overline{K_{w^{\prime}}^{\prime}} / K_{w^{\prime}}^{\prime}\right)}$, we see that $V^{\mathrm{Gal}\left(\bar{K} / K^{\prime}\right)}$ is also one dimensional.

Lemma 7.3. As in $\llbracket$, let $K$ be a finite extension of $\mathbb{Q}_{p}, \Gamma \subset \operatorname{PGL}_{d+1}(K)$ a cocompact torsion free discrete subgroup, and $K^{\prime}$ a finite extension of $K$ inside 
$\bar{K}$. Fix a prime number $l$, which is not necessarily different from $p$. Assume that $d$ is even. Then, the $\mathbb{Q}_{l}$-vector space

$$
H_{\text {ét }}^{d}\left(X_{\Gamma} \otimes_{K} \bar{K}, \mathbb{Q}_{l}\left(\frac{d}{2}\right)\right)^{\operatorname{Gal}\left(\bar{K} / K^{\prime}\right)}
$$

is one dimensional.

Proof. We put $V:=H_{\text {êt }}^{d}\left(X_{\Gamma} \otimes_{K} \bar{K}, \mathbb{Q}_{l}\right)$ for simplicity. Let $\mathbb{F}_{q^{\prime}}$ be the residue field of $K^{\prime}$.

Firstly, we consider the case $l \neq p$. As in 96.4 , we see that

$$
V^{I_{K^{\prime}}}=\mathbb{Q}_{l}^{\oplus \mu(\Gamma)} \oplus \mathbb{Q}_{l}\left(-\frac{d}{2}\right) .
$$

Recall that the monodromy filtration is invariant under a finite extension of the base field. Therefore,

$$
\left(V\left(\frac{d}{2}\right)\right)^{\mathrm{Gal}\left(\bar{K} / K^{\prime}\right)}=\left(V\left(\frac{d}{2}\right)^{I_{K^{\prime}}}\right)^{\mathrm{Fr}_{q^{\prime}}=1}=\left(\mathbb{Q}_{l}\left(\frac{d}{2}\right)^{\oplus \mu(\Gamma)} \oplus \mathbb{Q}_{l}\right)^{\mathrm{Fr}_{q^{\prime}}=1}=\mathbb{Q}_{l}
$$

is one dimensional, where " $\mathrm{Fr}_{q^{\prime}}=1$ " denotes the subspace consisting of elements fixed by the action of $\mathrm{Fr}_{q^{\prime}}$.

The case $l=p$ is similar but we work with $D_{\text {st }}(V)$ instead of $V$ itself. Let $m:=\operatorname{dim}_{\mathbb{Q}_{p}}\left(V\left(\frac{d}{2}\right)\right)^{\operatorname{Gal}\left(\bar{K} / K^{\prime}\right)}$. We know that $m \geq 1$. There is a $\operatorname{Gal}\left(\bar{K} / K^{\prime}\right)$ equivariant injection $\mathbb{Q}_{p}\left(-\frac{d}{2}\right)^{\oplus m} \hookrightarrow V$. By taking Fontaine's functor $D_{\text {st }}$ for $\operatorname{Gal}\left(\bar{K} / K^{\prime}\right)$-representations, we have an injection $D_{\text {st }}\left(\mathbb{Q}_{p}\left(-\frac{d}{2}\right)\right)^{\oplus m} \hookrightarrow D_{\text {st }}(V)$, which is compatible with the actions of $N, \varphi$. Since $N=0$ on $D_{\text {st }}\left(\mathbb{Q}_{p}\left(-\frac{d}{2}\right)\right)$, the image must be contained in $D_{\text {st }}(V)^{N=0}$. Since $\varphi$ acts on $D_{\text {st }}\left(\mathbb{Q}_{p}\left(-\frac{d}{2}\right)\right)$ via multiplication by $q^{d}, D_{\mathrm{st}}(V)^{N=0}$ has a subspace of dimension $m$ where $\varphi$ acts via multiplication by $q^{d}$. On the other hand, as we see in 46.6 , the dimension of such a subspace is at most 1 . Hence we have $m=1$.

Hence the assertion of Lemma 7.3 is proved, and the proof of Theorem 7.1 is complete.

Remark 7.4. It is known that certain Shimura varieties admit $p$-adic uniformization (for example, see [RZ2], Theorem 6.50, Corollary 6.51). Therefore, we can apply Theorem [7.1 to such Shimura varieties. Moreover, since the Hasse-Weil zeta functions of them can be written in terms of automorphic $L$-functions, it seems possible to deduce another form of the Tate conjecture for them concerning the orders of poles of the Hasse-Weil zeta functions (see [Ta], Conjecture 2).

Remark 7.5. In the function field case, Theorem [7.1 was essentially obtained by Laumon-Rapoport-Stuhler by the same method as above ([LRS, Proposition 16.2). Note that, in the function field case, the weight-monodromy conjecture was already known to hold by Deligne ([De3] $)$. 


\section{REFERENCES}

[AdS] G. Alon, E. de Shalit, Cohomology of discrete groups in harmonic cochains on buildings Israel J. Math. 135 (2003), 355-380.

[Be] V. G. Berkovich, Étale cohomology for non-Archimedean analytic spaces, Inst. Hautes Études Sci. Publ. Math. No. 78 (1993), 5-161.

[Č] I. V. Čerednik, Uniformization of algebraic curves by discrete arithmetic subgroups of $\mathrm{PGL}_{2}\left(k_{w}\right)$ with compact quotient spaces, Mat. Sb. (N.S.) 100(142) (1976), no. 1, 59-88, 165.

[CLS] B. Chiarellotto, B. Le Stum, Sur la pureté de la cohomologie cristalline, C. R. Acad. Sci. Paris Sér. I Math. 326 (1998), no. 8, 961-963.

[De1] P. Deligne, Théorie de Hodge I, in Actes du Congrès International des Mathématiciens (Nice, 1970), Tome 1, 425-430, Gauthier-Villars, Paris, 1971.

[De2] P. Deligne, La conjecture de Weil I, Inst. Hautes Études Sci. Publ. Math. No. 43, (1974), 273-307.

[De3] P. Deligne, La conjecture de Weil II, Inst. Hautes Études Sci. Publ. Math. No. 52, (1980), $137-252$.

[Dr] V. G. Drinfeld, Coverings of p-adic symmetric domains, Funkcional. Anal. i Priložen. 10 (1976), no. 2, 29-40.

[Fo] J.-M. Fontaine, Représentations p-adiques semi-stables (with an appendix by Pierre Colmez), Périodes p-adiques (Bures-sur-Yvette, 1988), Astérisque No. 223 (1994), 113184.

[Fu] W. Fulton, Intersection theory, Second edition, Springer, Berlin, 1998.

[Ga] H. Garland, p-adic curvature and the cohomology of discrete subgroups of p-adic groups, Ann. of Math. (2) 97 (1973), 375-423.

[GM] H. Gillet, W. Messing, Cycle classes and Riemann-Roch for crystalline cohomology, Duke Math. J. 55 (1987), no. 3, 501-538.

[Gr] M. Gros, Classes de Chern et classes de cycles en cohomologie de Hodge-Witt logarithmique, Mém. Soc. Math. France (N.S.) No. 21 (1985).

[GK] E. Große-Klönne, On the de Rham and Crystalline Cohomology of $\Omega_{K}^{(d+1)}$, preprint, 2001.

[GNA] F. Guillén, V. Navarro Aznar, Sur le théorème local des cycles invariants Duke Math. J. 61 (1990), no. 1, 133-155.

[Harr] M. Harris, On the local Langlands correspondence, Proceedings of the International Congress of Mathematics, Beijing, 2002.

[Hart1] R. Hartshorne, Ample subvarieties of algebraic varieties, Lecture Notes in Mathematics, Vol. 156, Springer-Verlag, Berlin-New York 1970.

[Hart2] R. Hartshorne, Algebraic geometry, Graduate Texts in Mathematics, No. 52. SpringerVerlag, New York-Heidelberg, 1977.

[HK] O. Hyodo, K. Kato, Semi-stable reduction and crystalline cohomology with logarithmic poles, Périodes p-adiques (Bures-sur-Yvette, 1988), Astérisque No. 223 (1994), 221-268.

[Il1] L. Illusie, Réalisation l-adique de l'accouplement de monodromie d'après A. Grothendieck, Courbes modulaires et courbes de Shimura (Orsay, 1987/1988), Astérisque No. 196-197 (1991), 27-44.

[Il2] L. Illusie, Autour du théorème de monodromie locale, Périodes p-adiques (Bures-surYvette, 1988), Astérisque No. 223, (1994), 9-57.

[Il3] L. Illusie, An overview of the work of K. Fujiwara, K. Kato, and C. Nakayama on logarithmic etale cohomology, in Cohomologies p-adiques et applications arithmétiques, II, Astérisque No. 279 (2002), 271-322.

[It1] T. Ito, Weight-monodromy conjecture over equal characteristic local fields, math.NT/0308141, 2003.

[It2] T. Ito, Weight-monodromy conjecture for certain threefolds in mixed characteristic, Internat. Math. Res. Notices 2004, no. 2, 69-87. 
[dJ] A. J. de Jong, Smoothness, semi-stability and alterations, Inst. Hautes Études Sci. Publ. Math. No. 83, (1996), 51-93.

[dJvdP] J. de Jong, M. van der Put, Étale cohomology of rigid analytic spaces, Doc. Math. 1 (1996), No. 01, 1-56.

[Ka] K. Kato, Semi-stable reduction and p-adic étale cohomology, Périodes p-adiques (Buressur-Yvette, 1988), Astérisque No. 223 (1994), 269-293.

$[\mathrm{KM}]$ N. M. Katz, W. Messing, Some consequences of the Riemann hypothesis for varieties over finite fields, Invent. Math. 23 (1974), 73-77.

[Kl] S. L. Kleiman, The standard conjectures, in Motives (Seattle, WA, 1991), 3-20, Proc. Sympos. Pure Math., Part 1, Amer. Math. Soc., Providence, RI, 1994.

$[\mathrm{Ku}] \mathrm{A}$. Kurihara, Construction of p-adic unit balls and the Hirzebruch proportionality, Amer. J. Math. 102 (1980), no. 3, 565-648.

[LRS] G. Laumon, M. Rapoport, U. Stuhler, $\mathscr{D}$-elliptic sheaves and the Langlands correspondence, Invent. Math. 113 (1993), no. 2, 217-338.

[Mc] P. McMullen, On simple polytopes, Invent. Math. 113 (1993), no. 2, 419-444.

[Mo] A. Mokrane, La suite spectrale des poids en cohomologie de Hyodo-Kato, Duke Math. J. 72 (1993), no. 2, 301-337.

[Mum] D. Mumford, An analytic construction of degenerating curves over complete local rings, Compositio Math. 24 (1972), 129-174.

[Mus] G. A. Mustafin, Non-archimedean uniformization, Math. USSR-Sb. 34 (1978), no. 2, $187-214$.

[O] T. Ochiai, l-independence of the trace of monodromy, Math. Ann. 315 (1999), no. 2, 321340.

[Rap] M. Rapoport, On the bad reduction of Shimura varieties, in Automorphic forms, Shimura varieties, and L-functions, Vol. II (Ann Arbor, MI, 1988), 253-321, Academic Press, Boston, MA, 1990.

[RZ1] M. Rapoport, T. Zink, Über die lokale Zetafunktion von Shimuravarietäten. Monodromiefiltration und verschwindende Zyklen in ungleicher Charakteristik, Invent. Math. 68 (1982), no. 1, 21-101.

[RZ2] M. Rapoport, T. Zink, Period spaces for p-divisible groups, Ann. of Math. Stud., 141, Princeton Univ. Press, Princeton, NJ, 1996.

[RX] W. Raskind, X. Xarles, On the étale cohomology of algebraic varieties with totally degenerate reduction over p-adic fields, math.NT/0306123 2003.

[Ray] M. Raynaud, Géométrie analytique rigide d'après Tate, Kiehl,..., Bull. Soc. Math. France, Mém. 39-40, 319-327, (1974).

[SaM1] M. Saito, Modules de Hodge polarisables, Publ. Res. Inst. Math. Sci. 24 (1988), no. 6, 849-995.

[SaM2] M. Saito, Monodromy filtration and positivity, math.AG/0006162 2000.

[SaT] T. Saito, Modular forms and p-adic Hodge theory, Invent. Math. 129 (1997), no. 3, 607-620.

[SS] P. Schneider, U. Stuhler, The cohomology of p-adic symmetric spaces, Invent. Math., 105, (1991), no. 1, 47-122.

[Se1] J.-P. Serre, Corps locaux, Deuxieme edition, Hermann, Paris, 1968.

[Se2] J.-P. Serre, Facteurs locaux des fonctions zêta des variétés algébriques (définitions et conjectures), Sem. Delange-Pisot-Poitou, 1970.

[ST] J.-P. Serre, J. Tate, Good reduction of abelian varieties, Ann. of Math. (2) 88 (1968), $492-517$.

[dS] E. de Shalit, The p-adic monodromy-weight conjecture for p-adically uniformized varieties, preprint, 2003, to appear in Compositio Math.

[St] J. Steenbrink, Limits of Hodge structures, Invent. Math. 31 (1975/76), no. 3, 229-257.

[Ta] J. Tate, Algebraic cycles and poles of zeta functions, in Arithmetical Algebraic Geometry (Proc. Conf. Purdue Univ., 1963), 93-110, Harper \& Row, New York, 1965. 
[Te] T. Terasoma, Monodromy weight filtration is independent of l, math.AG/9802051, 1998.

[Ts] T. Tsuji, $p$-adic étale cohomology and crystalline cohomology in the semi-stable reduction case, Invent. Math. 137 (1999), no. 2, 233-411.

[Va] Y. Varshavsky, p-adic uniformization of unitary Shimura varieties, Inst. Hautes Études Sci. Publ. Math. No. 87, (1998), 57-119.

[We] A. Weil, Introduction à l'étude des variétés kählériennes, Hermann, Paris, 1958.

[SGA4-III] Théorie des topos et cohomologie étale des schémas. Tome 3, Lecture Notes in Math., 305, Springer, Berlin, 1973.

[SGA5] Cohomologie l-adique et fonctions L, Lecture Notes in Math., 589, Springer, Berlin, 1977.

[SGA7-I] Groupes de monodromie en géométrie algébrique. I, Lecture Notes in Math., 288, Springer, Berlin, 1972.

Department of Mathematics, Faculty of Science, Kyoto University, Kyoto 606-8502, JAPAN

E-mail address: tetsushi@math.kyoto-u.ac.jp 